\title{
Engaging Alkenes and Alkynes in Deaminative Alkyl-Alkyl and Alkyl-Vinyl Cross-Couplings of Alkylpyridinium Salts
}

Kristen M. Baker, Diana Lucas Baca, Shane L. Plunkett, Mitchell E. Daneker, Mary P. Watson*

Department of Chemistry and Biochemistry, University of Delaware, Newark, DE 19716

mpwatson@udel.edu

\section{Supplementary Information}

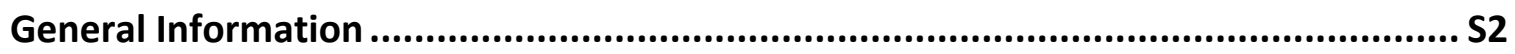

High-Throughput Experimentation (HTE) Studies .................................................. S3

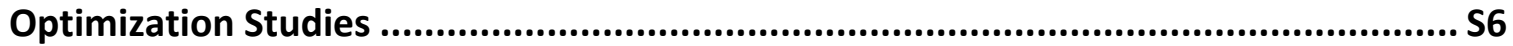

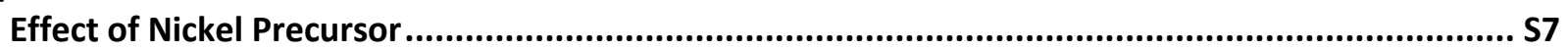

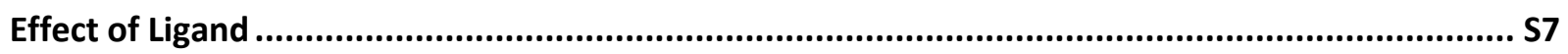

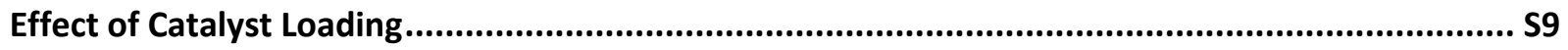

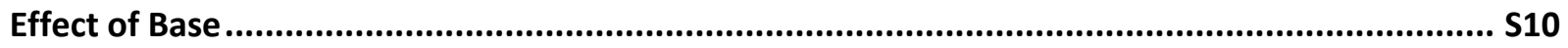

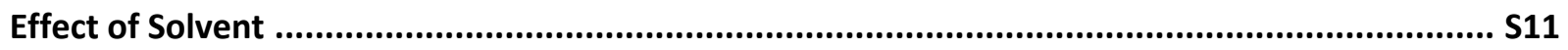

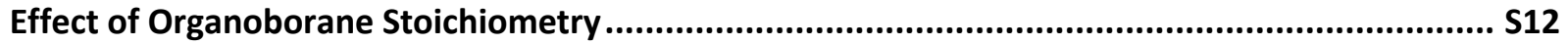

Effect of Stirring KF with Solution of 9-BBN and Alkene......................................................... S13

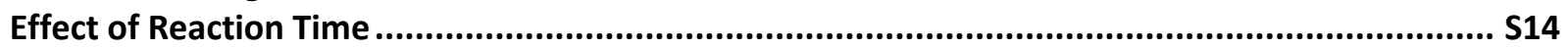

Cross-Couplings of Pyridinium Salts and Alkenes and Alkynes via Organoboranes ... S14

General Procedure A: Alkylation of Alkyl Pyridinium Salts with Alkenes..................................... S14

General Procedure B: Vinylation of Alkyl Pyridinium Salts with Alkynes...................................... S24

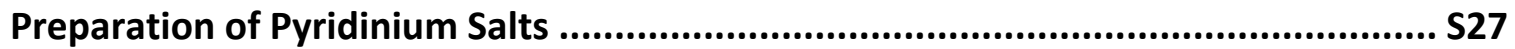

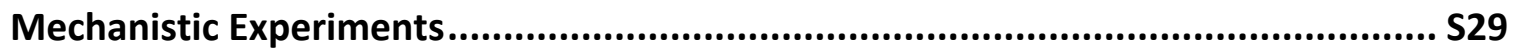

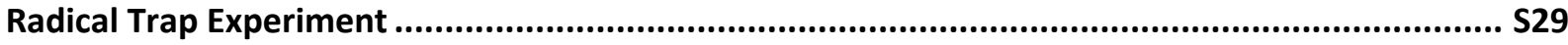

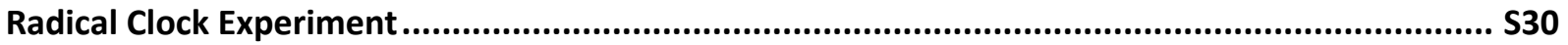

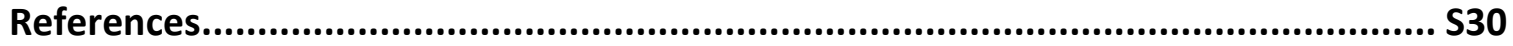

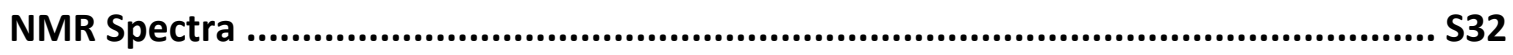




\section{General Information}

Reactions were performed in oven-dried Schlenk flasks or in oven-dried, round-bottomed flasks unless otherwise noted. Round-bottomed flasks were fitted with rubber septa, and reactions were conducted under an atmosphere of $\mathrm{N}_{2}$. Stainless steel syringes or cannulae were used to transfer air- and moisture-sensitive liquids. Flash chromatography was performed on silica gel 60 (40-63 $\mu \mathrm{m}, 60 \AA$ ) unless otherwise noted. Commercial reagents, including 2,4,6-triphenylpyrylium tetrafluoroborate, were purchased from Sigma Aldrich, Acros, AstaTech, Fisher, Strem, TCI, Combi Blocks, Alfa Aesar, AK Scientific, Bide Pharmatech, Oakwood, or Cambridge Isotopes Laboratories and used as received with the following exceptions: $\mathrm{MeCN}$ and $\mathrm{CH}_{2} \mathrm{Cl}_{2}$ were dried by passing through drying columns. ${ }^{1} \mathrm{MeCN}$ was then degassed by sparging with $\mathrm{N}_{2}$. Oven-dried potassium carbonate was added to $\mathrm{CDCl}_{3}$ to remove trace acid. Potassium fluoride for small scale cross-couplings was dried in the oven overnight at $115^{\circ} \mathrm{C}$, passed through a sieve (U.S. Standard Test Sieve, E-11 Standard, No. 200) to ensure uniformity throughout the powder, and then stored in a desiccator. $4 \AA$ Molecular sieves were purchased and heated at $200{ }^{\circ} \mathrm{C}$ under vacuum, then crushed and stored in a desiccator. Proton nuclear magnetic resonance ( ${ }^{1} \mathrm{H}$ NMR) spectra, carbon nuclear magnetic resonance $\left({ }^{13} \mathrm{C} \mathrm{NMR}\right)$ spectra, and fluorine nuclear magnetic resonance spectra $\left({ }^{19} \mathrm{~F}\right.$ NMR) were recorded on both $400 \mathrm{MHz}$ and $600 \mathrm{MHz}$ spectrometers. Chemical shifts for protons are reported in parts per million downfield from tetramethylsilane and are referenced to residual protium in the NMR solvent $\left(\mathrm{CHCl}_{3}=\delta 7.26\right)$. Chemical shifts for carbon are reported in parts per million downfield from tetramethylsilane and are referenced to the carbon resonances of the solvent $\left(\mathrm{CDCl}_{3}=\delta 77.16\right)$. Chemical shifts for fluorine were externally referenced to $\mathrm{CFCl}_{3}$ in $\mathrm{CDCl}_{3}\left(\mathrm{CFCl}_{3}=\delta 0\right)$. Data are represented as follows: chemical shift, multiplicity (br = broad, $\mathrm{s}$ $=$ singlet, $\mathrm{d}=$ doublet, $\mathrm{t}=$ triplet, $\mathrm{q}=$ quartet, $\mathrm{p}=$ pentet, $\mathrm{m}=$ multiplet, $\mathrm{dd}=$ doublet of doublets, $\mathrm{ddd}=$ doublet of doublets of doublets, $\mathrm{dt}=$ doublet of triplets, $\mathrm{dq}=$ doublet of quartets, $\mathrm{dp}=$ doublet of pentets, $\mathrm{tt}=$ triplet of triplets, $\mathrm{td}=$ triplet of doublets, $\mathrm{h}=$ heptet), coupling constants in Hertz $(\mathrm{Hz})$, integration. Infrared (IR) spectra were obtained using FTIR spectrophotometers with material loaded onto a $\mathrm{KBr}$ plate. The mass spectral data were obtained at the University of Delaware mass spectrometry facility. Melting points were taken on a Thomas-Hoover Uni-Melt Capillary Melting Point Apparatus. 


\section{High-Throughput Experimentation (HTE) Studies}
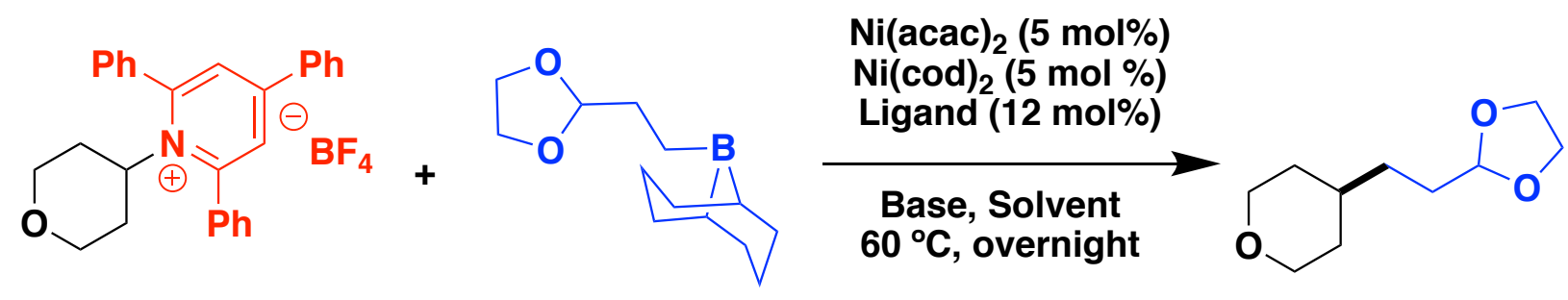

Representative Preparation of Alkylborane. According to literature procedure, 2-vinyl1,3-dioxalane ( $0.50 \mathrm{~mL}, 5.0 \mathrm{mmol}, 1.0$ equiv) and 9-borabicyclo[3.3.1]nonane (9-BBN, $0.5 \mathrm{M}$ in THF, $10 \mathrm{~mL}, 5.0 \mathrm{mmol}, 1.0$ equiv) were combined and stirred in a bomb at room temperature under a $\mathrm{N}_{2}$ atmosphere. ${ }^{2}$ The bomb was then sealed with a Teflon stopper, and moved into a $\mathrm{N}_{2-}$ atmosphere glovebox.

General HTE Procedure. In a $\mathrm{N}_{2}$-atmosphere glovebox, $250-\mu \mathrm{L}$ vials containing preplated ligands ( $1 \mu \mathrm{mol}$ of ligand in each, $12 \mathrm{~mol} \%)$ were charged with a stir bar and placed in a 96-well reaction plate. $\mathrm{Ni}(\mathrm{acac})_{2}(92 \mu \mathrm{g}, 0.42 \mu \mathrm{mol}, 5 \mathrm{~mol} \%), \mathrm{Ni}(\operatorname{cod})_{2}(117 \mu \mathrm{g}, 0.42 \mu \mathrm{mol}, 5 \mathrm{~mol}$ $\%$ ), and pyridinium salt 3a (4.0 mg, $8.3 \mu \mathrm{mol}, 1.0$ equiv) were added as stock solutions in $\mathrm{MeCN}$, such that the total amount of $\mathrm{MeCN}$ was $40 \mu \mathrm{L}$. Base was added to the solution of alkylborane. Then, a solution of alkylborane ( $0.5 \mathrm{M}$ in THF as described above, $50 \mu \mathrm{L}, 25 \mu \mathrm{mmol}, 3.0$ equiv) and base ( $28 \mu \mathrm{mol}, 3.3$ equiv) was added to each vial. The reaction plate was sealed, and the mixtures were stirred at $60{ }^{\circ} \mathrm{C}$ in an aluminum heating block overnight in the glovebox. The plate was then removed from the glovebox. A solution of 1,3,5-trimethoxybenzene (internal standard, $460 \mu \mathrm{g}, 2.8 \mu \mathrm{mol}, 0.33$ equiv) and $\mathrm{MeOH}(100 \mu \mathrm{L})$ was added to each vial. $30 \mu \mathrm{L}$ of each reaction mixture was then transferred to a second 96-well plate and diluted with $500 \mu \mathrm{L}$ of $\mathrm{MeOH}$. Using a centrifuge, any solids were deposited, before GC analysis. 

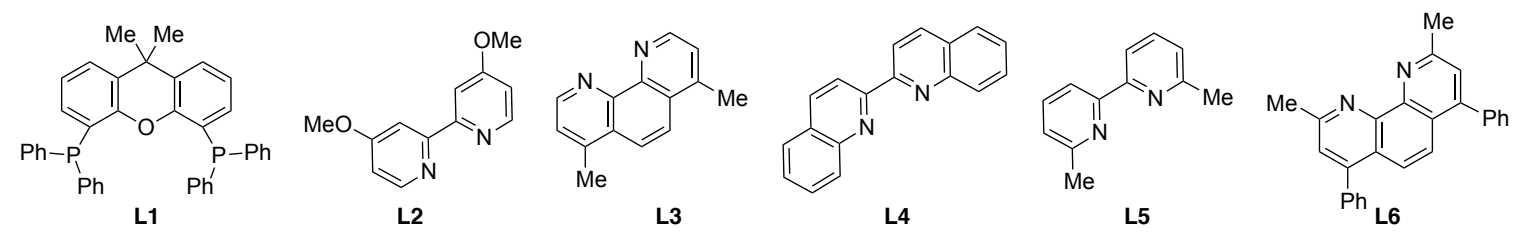

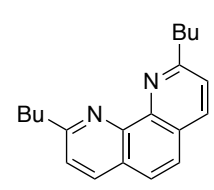

L7

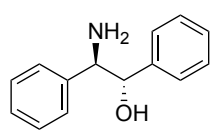

L13

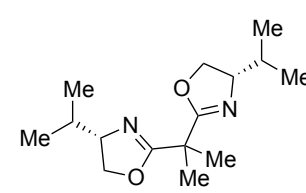

L8

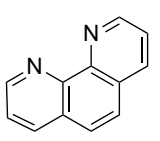

L14

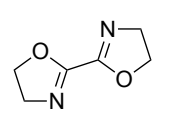

19<smiles>CNC1CCCC[C@H]1NC</smiles>

L15

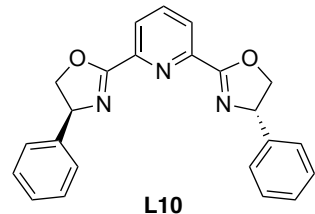

L10<smiles>c1ccc(CN[C@@H]2CCCC[C@H]2NCc2ccccc2)cc1</smiles>

L16<smiles>Cc1cnc2c(ccc3c(C)c(C)c(C)c(C)c32)c1C</smiles>

L11

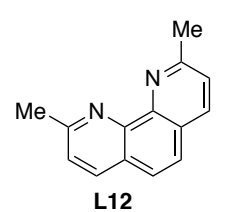

L17<smiles>N[C@@H]1CCCC[C@H]1N</smiles>

L18<smiles>CC(C)(C)c1ccnc(-c2cc(C(C)(C)C)cc(-c3cc(C(C)(C)C)ccn3)n2)c1</smiles>

L19<smiles>C1=Nc2c(-c3ccccc3)ccc(c2-c2ccccc2)C(c2ccccc2)=C1</smiles>

L25<smiles>CC(C)(C)c1ccnc(-c2cc(C(C)(C)C)ccn2)c1</smiles>

L31
L20<smiles>Clc1ccnc2c1ccc1c(Cl)ccnc12</smiles><smiles>Cc1ccnc(-c2cc(C)ccn2)c1</smiles>

L32
L21

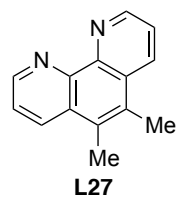

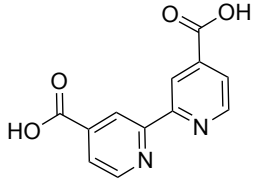

L33<smiles>O=C1C(=O)c2cccnc2-c2ncccc21</smiles><smiles>c1cnc2c(c1)c1nccnc1c1nccnc21</smiles>

L23<smiles>COc1ccnc2c1ccc1c(OC)ccnc12</smiles><smiles>Nc1ccnc(-c2cc(N)ccn2)c1</smiles>

L34<smiles>c1ccc(-c2ccnc(-c3cc(-c4ccccc4)ccn3)c2)cc1</smiles>

L35

Figure S-1. Ligands used in HTE studies. 


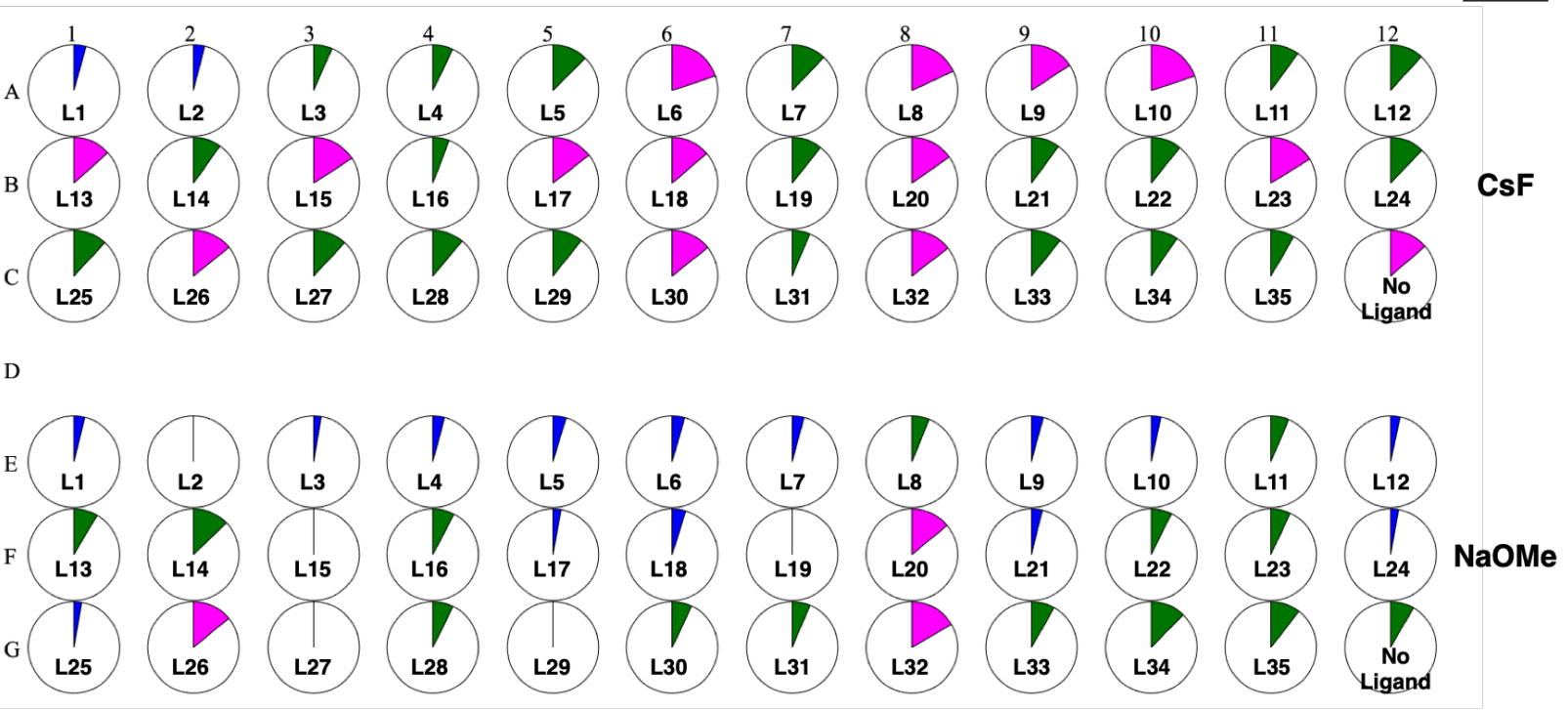

Figure S-2. HTE plate of 36 ligands vs. 2 bases. Ligand number denoted on each pie chart. Yields, as determined by GC analysis using 1,3,5-trimethoxybenzene as internal standard, are shown in pie charts for each reaction. In blue: $0-5 \%$ yield. In green: $5-13 \%$ yield. In magenta: $13-20 \%$ yield. 


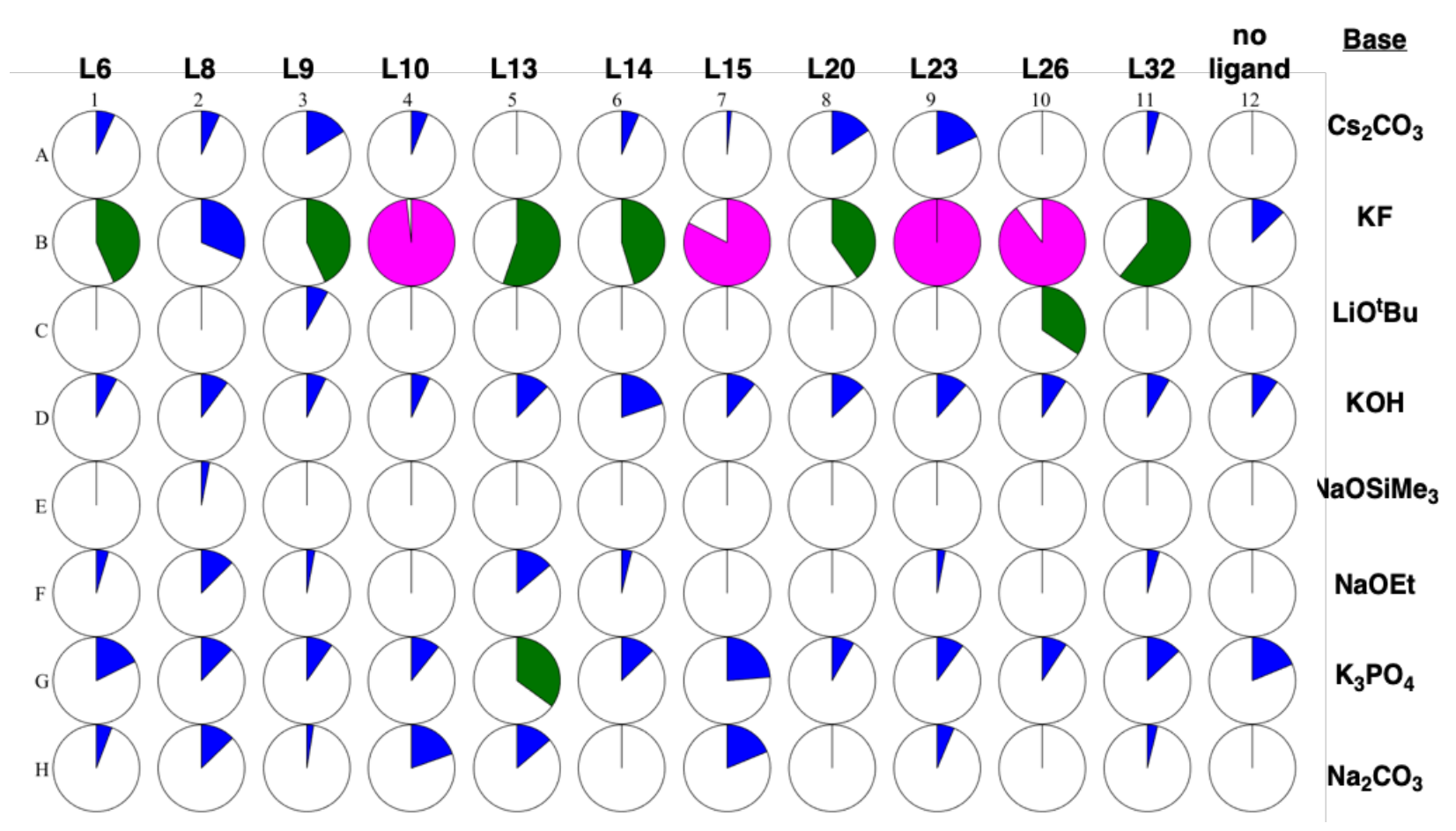

Figure S-3. HTE plate of 12 ligands vs. 8 bases. Yields, as determined by GC analysis using 1,3,5trimethoxybenzene as internal standard, are shown in pie charts for each reaction. In blue: 0-33\% yield. In green: $34-66 \%$ yield. In magenta: $67-100 \%$ yield.

\section{Optimization Studies}

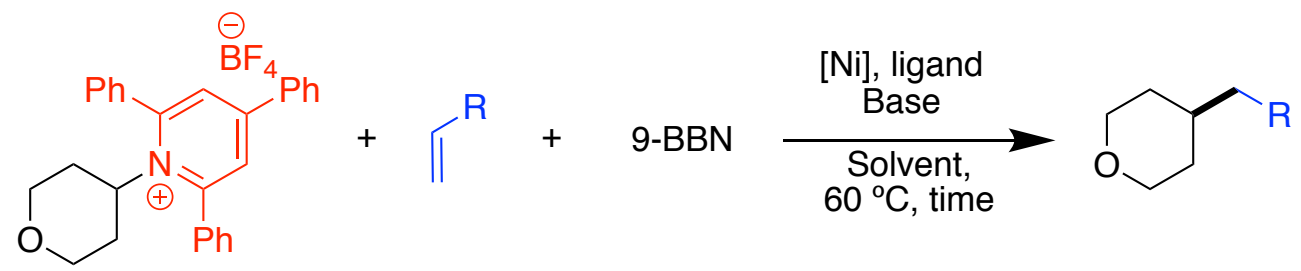

3a

General Optimization Procedure. In a $\mathrm{N}_{2}$-atmosphere glovebox, 9borabicyclo[3.3.1]nonane (9-BBN, 0.5 M in THF), KF, and alkene were stirred in an oven-dried 1-dram vial equipped with a stirbar at $80^{\circ} \mathrm{C}$ in an aluminum heating block for $30 \mathrm{~min}$. Meanwhile, nickel salt, ligand, and solvent were stirred for $15 \mathrm{~min}$. Pyridinium salt 3a (48.0 mg, $0.10 \mathrm{mmol}$, 1.0 equiv) was added to the vial containing 9-BBN. The nickel/ligand solution was added afterwards. The vial was capped with a Teflon-lined cap and removed from the glovebox. The 
mixture was stirred at $80{ }^{\circ} \mathrm{C}$ in an aluminum heating block for $24 \mathrm{~h}$, unless otherwise stated. The mixture was then diluted with $\mathrm{Et}_{2} \mathrm{O}(3 \mathrm{~mL})$ and filtered through a plug of silica gel, which was rinsed with $\mathrm{Et}_{2} \mathrm{O}(10 \mathrm{~mL})$. The filtrate was concentrated. 1,3,5-Trimethoxybenzene (internal standard) was added. $\mathrm{CDCl}_{3}$ was added, and the yield was determined by ${ }^{1} \mathrm{H}$ NMR analysis. Changes to this general procedure are noted in the tables below.

\section{Effect of Nickel Precursor}

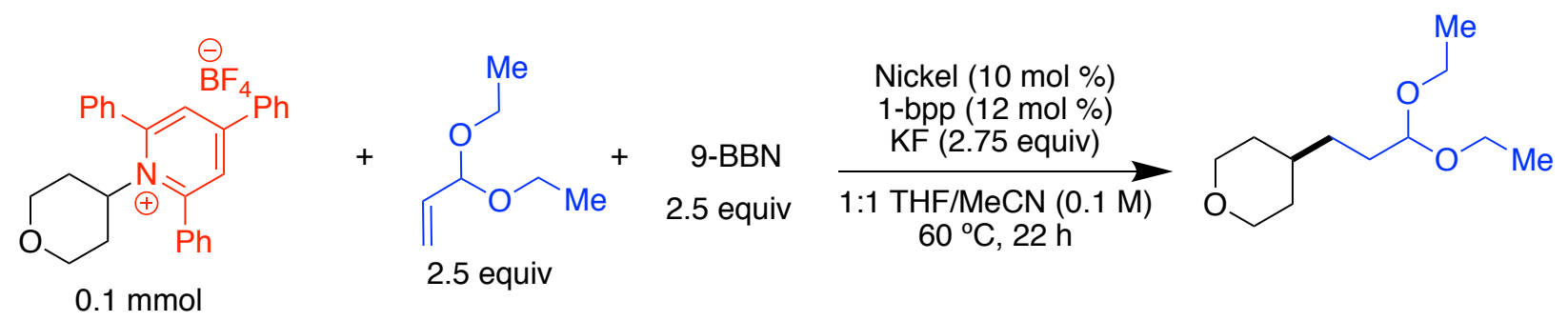

\begin{tabular}{ccc} 
entry & Nickel & yield $^{a}$ \\
\hline 1 & $\mathrm{NiCl}_{2} \cdot \mathrm{DME}$ & 65 \\
2 & $\mathrm{NiBr}_{2} \cdot \mathrm{DME}$ & 44 \\
3 & $\mathrm{NiI}_{2}$ & 76 \\
4 & $\mathrm{Ni}(\mathrm{acac})_{2}$ & 100 \\
5 & $\mathrm{NiCl}_{2} \cdot 4 \mathrm{H}_{2} \mathrm{O}$ & 95 \\
6 & $\mathrm{Ni}(\mathrm{OAc})_{2}$ & 28 \\
7 & $\mathrm{Ni}(\mathrm{OTf})_{2}$ & 68 \\
\hline
\end{tabular}

${ }^{a}$ Determined by ${ }^{1} \mathrm{H}$ NMR analysis using

1,3,5-trimethoxybenzene as internal standard.

\section{Effect of Ligand}<smiles></smiles>

$0.1 \mathrm{mmol}$

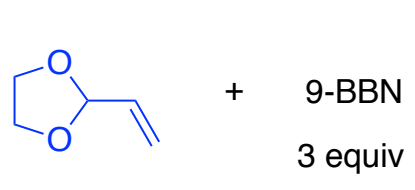

3 equiv

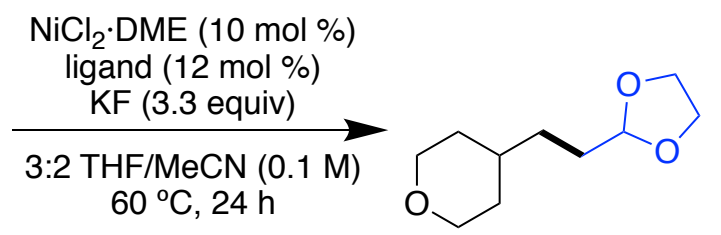




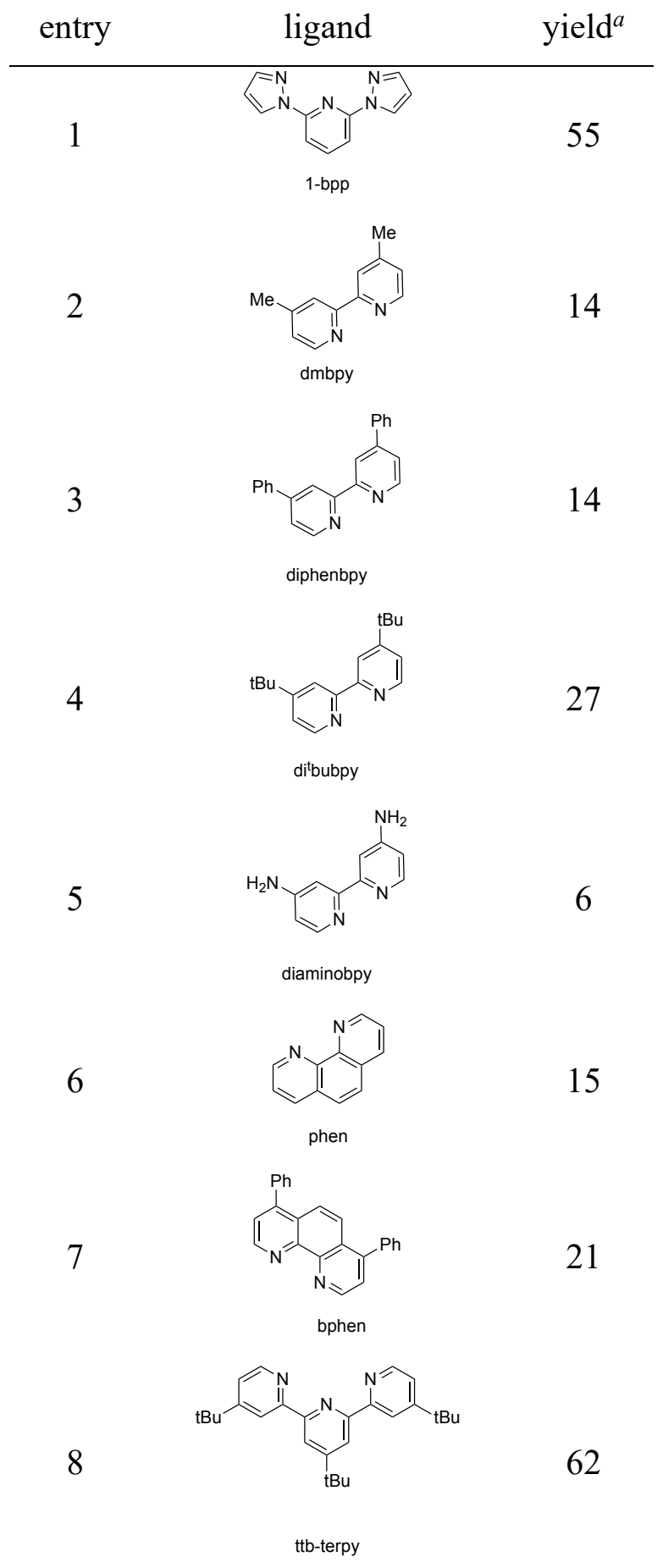

${ }^{a}$ Determined by ${ }^{1} \mathrm{H}$ NMR analysis using 1,3,5-trimethoxybenzene as internal standard. 


\section{Effect of Catalyst Loading}

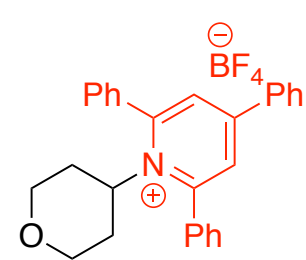

$0.1 \mathrm{mmol}$

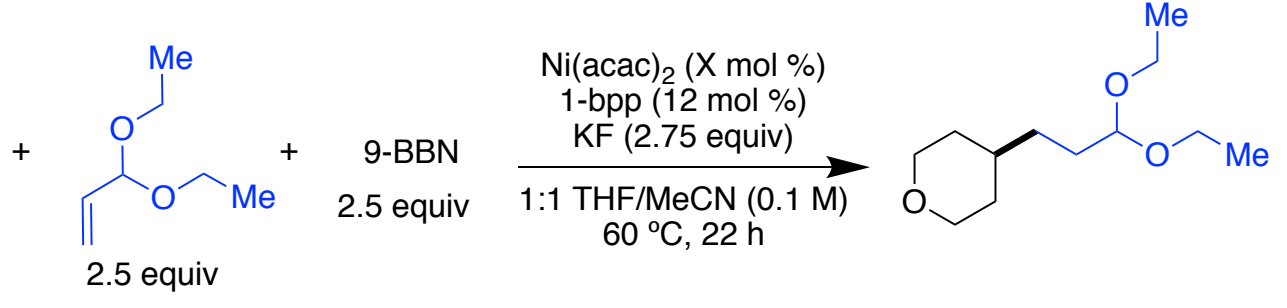

\begin{tabular}{ccc} 
entry & mol (\%) & yield $^{a}$ \\
\hline 1 & 2 & 50 \\
2 & 5 & 57 \\
3 & 10 & 80 \\
4 & 15 & 95 \\
5 & 20 & 99
\end{tabular}

a Determined by ${ }^{1} \mathrm{H}$ NMR analysis using 1,3,5-trimethoxybenzene as internal standard. 


\section{Effect of Base}

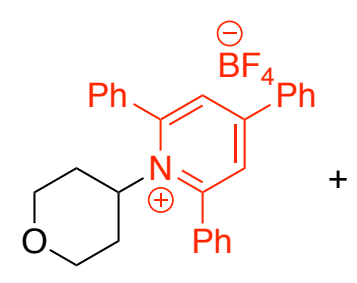

$0.1 \mathrm{mmol}$

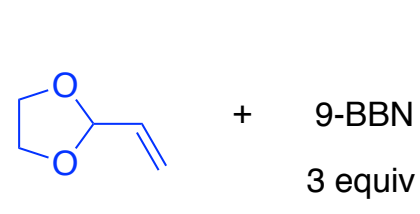

3 equiv
$\mathrm{NiCl}_{2} \cdot \mathrm{DME}(10 \mathrm{~mol} \%)$

1-bpp (12 mol \%)

$\underset{\substack{\text { 3HF/MeCN }(0.1 \mathrm{M}) \\ 60^{\circ} \mathrm{C}, 24 \mathrm{~h}}}{\text { Base }(3.3 \text { equiv) }}$

\begin{tabular}{ccc} 
entry & base & yield $^{a}$ \\
\hline 1 & $\mathrm{KF}$ (spray dried) & 75 \\
2 & $\mathrm{KF}$ (oven dried) & 68 \\
3 & $\mathrm{KF}$ & 18 \\
4 & $\mathrm{CsF}$ & 60 \\
5 & $\mathrm{KBr}$ & 0 \\
6 & $\mathrm{NaI}$ & 4 \\
7 & $\mathrm{AgF}$ & 0 \\
8 & $\mathrm{NaCl}$ & 0 \\
9 & $\mathrm{LiF}$ & 0
\end{tabular}

a Determined by ${ }^{1} \mathrm{H}$ NMR analysis using

1,3,5-trimethoxybenzene as internal standard. 


\section{Effect of Solvent}
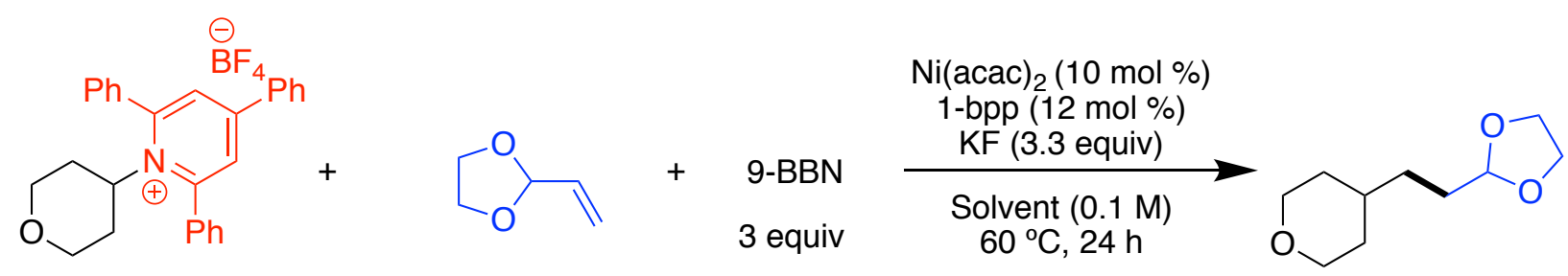

$0.1 \mathrm{mmol}$

\begin{tabular}{ccc}
$\begin{array}{c}3 \text { equiv } \\
\text { entry }\end{array}$ & solvent & yield $^{a}$ \\
\hline 1 & MeCN & 93 \\
2 & DMA & 21 \\
3 & DMF & 21 \\
4 & NMP & 31 \\
5 & Dioxane & 20
\end{tabular}

$a$ Determined by ${ }^{1} \mathrm{H}$ NMR analysis using 1,3,5trimethoxybenzene as internal standard. 
Effect of Organoborane Stoichiometry<smiles>[P-]c1cc(-c2ccccc2)[n+](C2CCOCC2)c(-c2ccccc2)c1</smiles>

$0.1 \mathrm{mmol}$

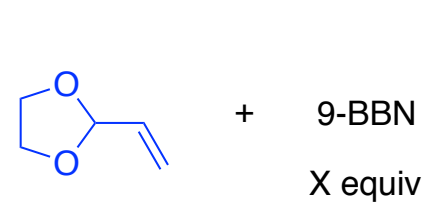

$\mathrm{X}$ equiv

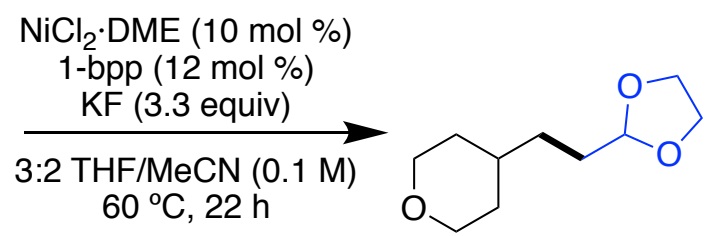

entry equiv of organoborane (X) yield $^{a}$

\begin{tabular}{lll}
\hline 1 & 2.5 & 49 \\
2 & 2.6 & 48 \\
3 & 2.7 & 47 \\
4 & 2.8 & 42 \\
5 & 2.9 & 46 \\
6 & 3.0 & 26 \\
7 & 3.1 & 15 \\
8 & 3.2 & 15 \\
9 & 3.3 & 28 \\
10 & 3.4 & 41 \\
11 & 3.5 & 15 \\
\hline
\end{tabular}

a Determined by ${ }^{1} \mathrm{H}$ NMR analysis using 1,3,5trimethoxybenzene as internal standard. 


\section{Effect of Stirring KF with Solution of 9-BBN and Alkene}

In these experiments, KF was stirred with the solution of $9-\mathrm{BBN}$ and alkene at $80{ }^{\circ} \mathrm{C}$ for the times indicated in the table below.

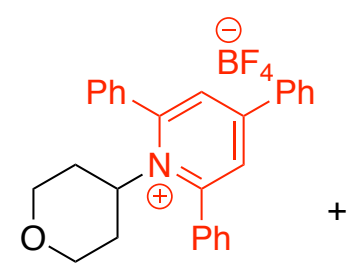

$0.1 \mathrm{mmol}$

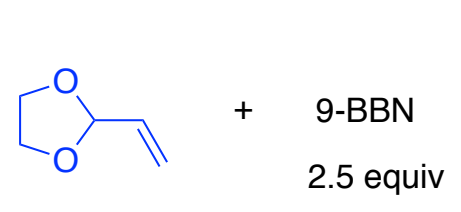

2.5 equiv

\begin{tabular}{ccc} 
entry & stirring time (min) & yield $^{a}$ \\
\hline 1 & 15 & 78 \\
2 & 30 & 93 \\
3 & 45 & 93 \\
4 & 60 & 83
\end{tabular}

a Determined by ${ }^{1} \mathrm{H}$ NMR analysis using

1,3,5-trimethoxybenzene as internal standard. 
Effect of Reaction Time<smiles></smiles>

$0.1 \mathrm{mmol}$

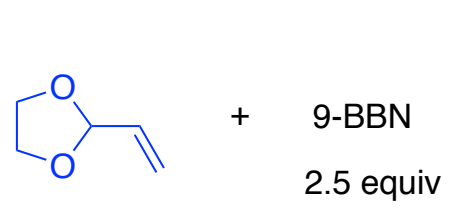

2.5 equiv
$\mathrm{NiCl}_{2} \cdot \mathrm{DME}(10 \mathrm{~mol} \%)$

1 -bpp (12 mol \%)

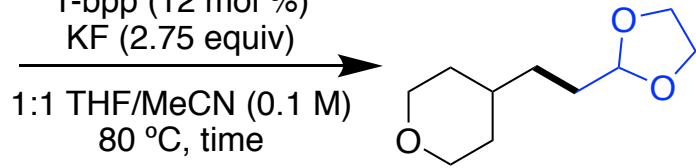

$80^{\circ} \mathrm{C}$, time

yield $^{a}$

\begin{tabular}{ccc} 
entry & reaction time (h) & yield $^{a}$ \\
\hline 1 & 1 & 6 \\
2 & 2 & 10 \\
3 & 4 & 15 \\
4 & 18 & 86 \\
5 & 20 & 80 \\
6 & 22 & 100 \\
7 & 24 & 98
\end{tabular}

a Determined by ${ }^{1} \mathrm{H}$ NMR analysis using

1,3,5-trimethoxybenzene as internal standard.

\section{Cross-Couplings of Pyridinium Salts and Alkenes and Alkynes via}

\section{Organoboranes}

\section{General Procedure A: Alkylation of Alkyl Pyridinium Salts with Alkenes}

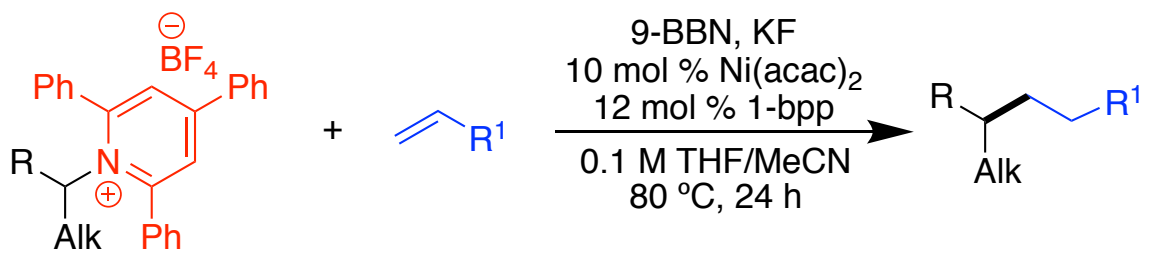

An oven-dried, 25-mL Schlenk flask equipped with a mechanical stirbar was charged with KF (160 mg, $2.75 \mathrm{mmol}, 2.75$ equiv) and alkene (2.5 mmol, 2.5 equiv), if solid. The flask was fitted with a rubber septum, sealed with parafilm, and evacuated and backfilled with nitrogen five times. 9-BBN (0.5 M solution in THF, $2.5 \mathrm{mmol}, 2.5$ equiv, $0.50 \mathrm{~mL})$ and alkene, if liquid, were added via syringe. The mixture was stirred and heated at $80^{\circ} \mathrm{C}$ in an oil bath for $30 \mathrm{~min}$. A second oven-dried, 10-mL Schlenk flask equipped with a mechanical stirbar was charged with $\mathrm{Ni}(\mathrm{acac})_{2}$ 
(2.5 mg, $0.10 \mathrm{mmol}, 0.10$ equiv), 1-bpp ( $2.6 \mathrm{mg}, 0.12 \mathrm{mmol}, 0.12$ equiv), and pyridinium salt (1.0 mmol, 1.0 equiv). The flask was fitted with a rubber septum, and evacuated and backfilled with nitrogen five times. Acetonitrile $(3 \mathrm{~mL})$ was added, and the mixture was stirred for $15 \mathrm{~min}$ at room temperature. The catalyst solution was then transferred to the $25-\mathrm{mL}$ Schlenk flask via syringe, rinsing with acetonitrile $(2 \mathrm{~mL})$, and then the mixture was stirred at $80^{\circ} \mathrm{C}$ in an oil bath for $24 \mathrm{~h}$. The mixture was allowed to cool to room temperature. For nonpolar products that might co-elute with organoborane species, $\mathrm{H}_{2} \mathrm{O}_{2}(0.4 \mathrm{~mL})$ was added, and the mixture was vigorously stirred for 5 min to oxidize the boron species. For polar products, this oxidation step was skipped. The aqueous layer was washed with EtOAc $(3 \times 20 \mathrm{~mL})$, dried $\left(\mathrm{MgSO}_{4}\right)$, filtered through a short pad of silica gel, and concentrated. The cross-coupled product was then purified via silica gel chromatography.

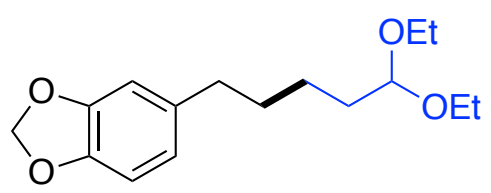

5-(5,5-Diethoxypentyl)-2H-1,3-benzodioxole (7). Prepared via General Procedure A using pyridinium salt 3b. During work-up, the oxidation step with $\mathrm{H}_{2} \mathrm{O}_{2}$ was used. The crude mixture was purified by silica gel chromatography $\left(2 \% \rightarrow 20 \% \mathrm{Et}_{2} \mathrm{O} /\right.$ hexanes) to give 7 (run 1: $149 \mathrm{mg}$, 52\%; run 2: $164 \mathrm{mg}, 58 \%)$ as an orange oil: ${ }^{1} \mathrm{H}$ NMR (600 MHz, Chloroform- $d$ ) $\delta 6.71$ (d, $J=7.9$ $\mathrm{Hz}, 1 \mathrm{H}), 6.66(\mathrm{~d}, J=1.7 \mathrm{~Hz}, 1 \mathrm{H}), 6.63-6.59(\mathrm{~m}, 1 \mathrm{H}), 5.90$ (s, 2H), $4.47(\mathrm{t}, J=5.7 \mathrm{~Hz}, 1 \mathrm{H}), 3.63$ (dq, $J=9.4,7.1 \mathrm{~Hz}, 2 \mathrm{H}), 3.48(\mathrm{dq}, J=9.4,7.0 \mathrm{~Hz}, 2 \mathrm{H}), 2.58-2.46(\mathrm{~m}, 2 \mathrm{H}), 1.68-1.52(\mathrm{~m}, 4 \mathrm{H})$, $1.43-1.34(\mathrm{~m}, 2 \mathrm{H}), 1.20(\mathrm{t}, J=7.1 \mathrm{~Hz}, 6 \mathrm{H}) ;{ }^{13} \mathrm{C}$ NMR (101 MHz, Chloroform- $d$ ) $\delta$ 147.6, 145.5, 136.6, 121.2 , 109.0, 108.2 103.0, 100.8, 61.1, 35.7, 33.6, 31.8, 24.4, 15.5; FTIR (neat) 2930, 1723, 1489, 1245, 1039, $809 \mathrm{~cm}^{-1}$; HRMS (LIFDI+) $[\mathrm{M}]^{+}$calculated for $\mathrm{C}_{16} \mathrm{H}_{24} \mathrm{O}_{4}: 280.1675$, found 280.1665 .

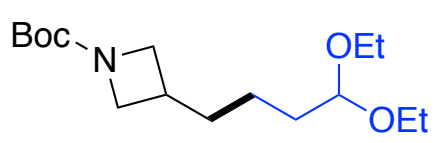

Tert-butyl 3-(4,4-diethoxybutyl)azetidine-1-carboxylate (8). Prepared via General Procedure A using pyridinium salt 3c. During work-up, the oxidation step with $\mathrm{H}_{2} \mathrm{O}_{2}$ was not used. The crude mixture was purified by silica gel chromatography $\left(10 \% \rightarrow 25 \% \rightarrow 50 \% \mathrm{Et}_{2} \mathrm{O} /\right.$ hexanes $)$ to give 8 (run 1: $198 \mathrm{mg}, 66 \%$; run 2: $187 \mathrm{mg}, 62 \%)$ as a yellow oil: ${ }^{1} \mathrm{H}$ NMR (400 MHz, 
Chloroform- $d$ ) $\delta 4.46(\mathrm{t}, J=5.7 \mathrm{~Hz}, 1 \mathrm{H}), 3.98(\mathrm{t}, J=8.3 \mathrm{~Hz}, 2 \mathrm{H}), 3.63(\mathrm{dq}, J=9.4,7.1 \mathrm{~Hz}, 2 \mathrm{H})$, $3.56-3.41(\mathrm{~m}, 4 \mathrm{H}), 2.46(\mathrm{tt}, J=7.9,5.5 \mathrm{~Hz}, 1 \mathrm{H}), 1.62-1.54(\mathrm{~m}, 4 \mathrm{H}), 1.43(\mathrm{~s}, 9 \mathrm{H}), 1.34-1.25$ (m, 2H), $1.20(\mathrm{t}, J=7.1 \mathrm{~Hz}, 6 \mathrm{H}) ;{ }^{13} \mathrm{C}$ NMR (101 MHz, Chloroform-d) $\delta 156.6,102.8,79.3,61.1$, 34.4, 33.5, 31.1, 29.0, 28.6, 22.3, 15.5; FTIR (neat) 2974, 2876, 2361, 1700, 1399, $1132 \mathrm{~cm}^{-1}$; HRMS (ESI+) $[\mathrm{M}+\mathrm{H}]^{+}$calculated for $\mathrm{C}_{16} \mathrm{H}_{32} \mathrm{NO}_{4}: 302.2331$, found 302.2326.

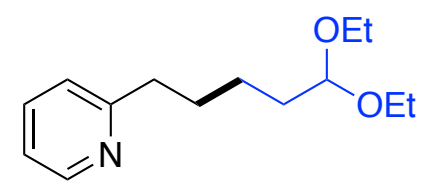

2-(5,5-Diethoxypentyl)pyridine (9). Prepared via General Procedure A using pyridinium salt 3d. During work-up, the oxidation step with $\mathrm{H}_{2} \mathrm{O}_{2}$ was not used. The crude mixture was purified by silica gel chromatography $(10 \% \rightarrow 20 \%$ EtOAc/hexanes) to give 9 (run 1: $154 \mathrm{mg}, 64 \%$; run 2: $169 \mathrm{mg}, 71 \%$ ) as a yellow oil: ${ }^{1} \mathrm{H}$ NMR $(600 \mathrm{MHz}$, Chloroform- $d) \delta 8.56(\mathrm{~s}, 1 \mathrm{H}), 7.59(\mathrm{t}, J=7.6$ $\mathrm{Hz}, 1 \mathrm{H}), 7.14(\mathrm{~m}, 2 \mathrm{H}), 4.47(\mathrm{t}, J=5.7 \mathrm{~Hz}, 1 \mathrm{H}), 3.62(\mathrm{dq}, J=9.4,7.0 \mathrm{~Hz}, 2 \mathrm{H}), 3.47$ (dq, $J=9.4$, $7.1 \mathrm{~Hz}, 2 \mathrm{H}), 2.79$ (t, $J=7.7 \mathrm{~Hz}, 2 \mathrm{H}), 1.76(\mathrm{~h}, J=7.6 \mathrm{~Hz}, 2 \mathrm{H}), 1.71-1.60$ (m, 2H), $1.52-1.40$ $(\mathrm{m}, 2 \mathrm{H}), 1.19(\mathrm{t}, J=7.1 \mathrm{~Hz}, 6 \mathrm{H}) ;{ }^{13} \mathrm{C}$ NMR (101 MHz, Chloroform- $\left.d\right) \delta 162.3,149.2,136.5,122.9$, 121.1, 102.9, 61.0, 38.4, 33.6, 29.9, 24.7, 15.9; FTIR (neat) 2974, 2929, 2864, 1434, 1128, 994, $749 \mathrm{~cm}^{-1}$; HRMS (ESI+) $[\mathrm{M}+\mathrm{H}]^{+}$calculated for $\mathrm{C}_{14} \mathrm{H}_{24} \mathrm{NO}_{2}: 238.1807$, found 238.1791 .<smiles>CCOC(=O)CCCCC(OCC)OCC</smiles>

Tert-butyl 6,6-diethoxyhexanoate (10). Prepared via General Procedure A using pyridinium salt 3e. During work-up, the oxidation step with $\mathrm{H}_{2} \mathrm{O}_{2}$ was used. The crude mixture was purified by silica gel chromatography $\left(10 \% \rightarrow 25 \% \mathrm{Et}_{2} \mathrm{O} /\right.$ hexanes) to give 10 (run 1: $138 \mathrm{mg}, 53 \%$; run 2: 159 mg, 61\%) as a light yellow oil: ${ }^{1} \mathrm{H}$ NMR (400 MHz, Chloroform- $d$ ) $\delta 4.47(\mathrm{t}, J=5.7 \mathrm{~Hz}, 1 \mathrm{H}), 3.63$ $(\mathrm{dq}, J=9.3,7.1 \mathrm{~Hz}, 2 \mathrm{H}), 3.48(\mathrm{dq}, J=9.4,7.0 \mathrm{~Hz}, 2 \mathrm{H}), 2.21(\mathrm{t}, J=7.5 \mathrm{~Hz}, 2 \mathrm{H}), 1.66-1.56(\mathrm{~m}$, 4H), $1.43(\mathrm{~s}, 9 \mathrm{H}), 1.41-1.30$ (m, 2H), 1.19 (t, $J=7.1 \mathrm{~Hz}, 6 \mathrm{H}) ;{ }^{13} \mathrm{C}$ NMR (101 MHz, Chloroformd) $\delta 173.2,102.8,80.2,61.0,35.6,33.4,28.2,25.1,24.4,15.5$; FTIR (neat) 2975, 2931, 1733, 1652, $1151 \mathrm{~cm}^{-1}$; HRMS (ESI+) $[\mathrm{M}+\mathrm{H}]^{+}$calculated for $\mathrm{C}_{14} \mathrm{H}_{29} \mathrm{O}_{4}:$ 261.2066, found 261.2187. 


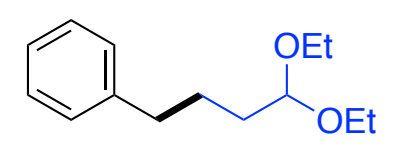

(4,4-Diethoxybutyl)benzene (11). Prepared via General Procedure A using pyridinium salt $3 \mathrm{f}$. During work-up, the oxiation step with $\mathrm{H}_{2} \mathrm{O}_{2}$ was used. The crude mixture was purified by silica gel chromatography $\left(5 \% \rightarrow 40 \% \mathrm{Et}_{2} \mathrm{O} /\right.$ hexanes) to give 11 (run 1: $211 \mathrm{mg}, 78 \%$; run 2: $222 \mathrm{mg}$, $85 \%$ ) as a light yellow oil: ${ }^{1} \mathrm{H}$ NMR (400 MHz, Chloroform- $d$ ) $\delta 7.39-7.30(\mathrm{~m}, 2 \mathrm{H}), 7.24(\mathrm{~s}, 3 \mathrm{H})$, $4.56(\mathrm{t}, J=5.2 \mathrm{~Hz}, 1 \mathrm{H}), 3.79-3.65(\mathrm{~m}, 2 \mathrm{H}), 3.54$ (tt, $J=8.6,6.5 \mathrm{~Hz}, 2 \mathrm{H}), 2.70$ (t, $J=7.0 \mathrm{~Hz}$, 2H), $1.88-1.64(\mathrm{~m}, 4 \mathrm{H}), 1.26(\mathrm{td}, J=7.1,1.3 \mathrm{~Hz}, 6 \mathrm{H}) ;{ }^{13} \mathrm{C}$ NMR (101 MHz, Chloroform- $d$ ) $\delta$ 142.5, 128.7, 128.4, 125.9, 103.0, 61.1, 35.9, 33.4, 26.8, 15.5; FTIR (neat) 2977, 2928, 1373, 1129 , 1063, $699 \mathrm{~cm}^{-1}$; HRMS (CI) [M-OEt] calculated for $\mathrm{C}_{12} \mathrm{H}_{17} \mathrm{O}: 177.1279$, found 177.1280.

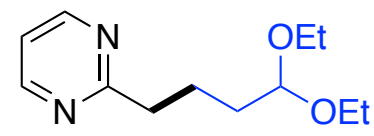

2-(4,4-Diethoxybutyl)pyrimidine (12). Prepared via General Procedure A using pyridinium salt 3g. During work-up, the oxdation step with $\mathrm{H}_{2} \mathrm{O}_{2}$ was not used. The crude mixture was purified by silica gel chromatography $(10 \% \rightarrow 50 \%$ EtOAc/hexanes) to give 12 (run 1: $142 \mathrm{mg}, 63 \%$; run 2: $153 \mathrm{mg}, 67 \%)$ as a yellow oil: ${ }^{1} \mathrm{H}$ NMR $(400 \mathrm{MHz}$, Chloroform- $d$ ) $\delta 8.66(\mathrm{~d}, J=4.9 \mathrm{~Hz}, 2 \mathrm{H})$, $7.12(\mathrm{t}, J=4.9 \mathrm{~Hz}, 1 \mathrm{H}), 4.53(\mathrm{t}, J=5.8 \mathrm{~Hz}, 1 \mathrm{H}), 3.63(\mathrm{dq}, J=9.4,7.1 \mathrm{~Hz}, 2 \mathrm{H}), 3.48(\mathrm{dq}, J=9.4$, $7.0 \mathrm{~Hz}, 2 \mathrm{H}), 2.99$ (t, $J=7.7 \mathrm{~Hz}, 2 \mathrm{H}), 1.96-1.84(\mathrm{~m}, 2 \mathrm{H}), 1.74-1.65(\mathrm{~m}, 2 \mathrm{H}), 1.19$ (t, $J=7.0 \mathrm{~Hz}$, $6 \mathrm{H}) ;{ }^{13} \mathrm{C}$ NMR (101 MHz, Chloroform- $d$ ) $\delta 171.3,157.1,118.6,102.8,61.0,39.3,33.3,24.1,15.5$; FTIR (neat) 2973, 2875, 1560, 1425, 1129, $1061 \mathrm{~cm}^{-1}$; HRMS (ESI+) $[\mathrm{M}+\mathrm{H}]^{+}$calculated for $\mathrm{C}_{12} \mathrm{H}_{21} \mathrm{~N}_{2} \mathrm{O}_{2}: 225.1603$, found 225.1596 .<smiles>CCOC(CC)CCCc1ccccc1-n1cccc1</smiles>

1-[2-(4,4-Diethoxybutyl)phenyl]-1H-pyrrole (13). Prepared via General Procedure A using pyridinium salt $3 \mathbf{h}$. During work-up, the oxidation step with $\mathrm{H}_{2} \mathrm{O}_{2}$ was not used. The crude mixture was purified by silica gel chromatography (25\% EtOAc/hexanes) to give 13 (run 1: $211 \mathrm{mg}, 74 \%$; run 2: $208 \mathrm{mg}, 73 \%$ ) as a dark red oil: ${ }^{1} \mathrm{H}$ NMR $(400 \mathrm{MHz}$, Chloroform- $d$ ) $\delta 7.31$ (dd, $J=4.1,2.0$ $\mathrm{Hz}, 2 \mathrm{H}), 7.26-7.22(\mathrm{~m}, 2 \mathrm{H}), 6.76(\mathrm{t}, J=2.1 \mathrm{~Hz}, 2 \mathrm{H}), 6.29(\mathrm{t}, J=2.1 \mathrm{~Hz}, 2 \mathrm{H}), 4.42-4.35(\mathrm{~m}$, $1 \mathrm{H}), 3.57$ (dq, $J=9.3,7.0 \mathrm{~Hz}, 2 \mathrm{H}), 3.42$ (dq, $J=9.3,7.0 \mathrm{~Hz}, 2 \mathrm{H}), 2.54-2.48$ (m, 2H), $1.56-1.48$ (m, 4H), $1.17(\mathrm{t}, J=7.0 \mathrm{~Hz}, 6 \mathrm{H}) ;{ }^{13} \mathrm{C}$ NMR (101 MHz, Chloroform- $d$ ) $\delta 140.4,138.5,130.1,127.9$, 
127.3, 126.7, 122.5, 108.8, 102.7, 61.0, 33.9, 30.9, 26.0, 15.5; FTIR (neat) 2973, 2928, 1717, 1502, 1065, $726 \mathrm{~cm}^{-1}$; HRMS (ESI-) [M-H-Et-OEt] $]^{-}$calculated for $\mathrm{C}_{14} \mathrm{H}_{14} \mathrm{NO}$ : 212.1076, found 212.1070 .

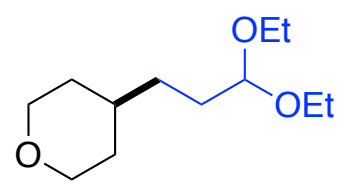

4-(3,3-Diethoxypropyl)oxane (6). Prepared via General Procedure A using pyridinium salt 3a. During work-up, the oxidation step with $\mathrm{H}_{2} \mathrm{O}_{2}$ was used. The crude mixture was purified by silica gel chromatography ( $1 \% \mathrm{MeOH}, 5 \% \mathrm{Et}_{2} \mathrm{O} /$ toluene) to give 6 (run 1: $203 \mathrm{mg}, 93 \%$; run 2: $198 \mathrm{mg}$, 91\%) as a light yellow oil: ${ }^{1} \mathrm{H}$ NMR (400 MHz, Chloroform- $d$ ) $\delta 4.46(\mathrm{t}, J=5.7 \mathrm{~Hz}, 1 \mathrm{H}), 4.00-$ 3.89 (m, 2H), 3.64 (dq, $J=9.4,7.1 \mathrm{~Hz}, 2 \mathrm{H}), 3.49$ (dq, $J=9.4,7.0 \mathrm{~Hz}, 2 \mathrm{H}), 3.36(\mathrm{td}, J=11.9,2.1$ $\mathrm{Hz}, 2 \mathrm{H}), 1.66-1.58$ (m, 4H), 1.46 (dddt, $J=14.5,10.4,6.9,3.7 \mathrm{~Hz}, 1 \mathrm{H}), 1.34-1.24$ (m, 4H), $1.20(\mathrm{t}, J=7.1 \mathrm{~Hz}, 6 \mathrm{H}) ;{ }^{13} \mathrm{C}$ NMR $(101 \mathrm{MHz}$, Chloroform- $d$ ) $\delta$ 103.2, 68.3, 61.1, 35.0, 33.7, 32.0, 30.7, 15.5. FTIR (neat) 2361, 2336, 1062, 667 $\mathrm{cm}^{-1}$; HRMS (ESI+) $[\mathrm{M}+\mathrm{H}]^{+}$calculated for $\mathrm{C}_{12} \mathrm{H}_{25} \mathrm{O}_{3}: 217.1804$, found 217.1377.

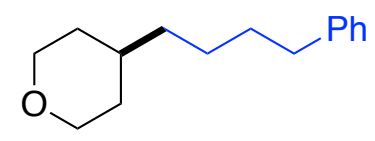

4-(4-Phenylbutyl)oxane (14). Prepared via General Procedure A using pyridinium salt 3a. During work-up, the oxidation step with $\mathrm{H}_{2} \mathrm{O}_{2}$ was used. The crude mixture was purified by silica gel chromatography $\left(5 \% \rightarrow 10 \% \mathrm{Et}_{2} \mathrm{O} /\right.$ hexanes) to give 14 (run 1: $220 \mathrm{mg}, 47 \%$; run 2: $218 \mathrm{mg}, 46 \%$ ) as a yellow oil: ${ }^{1} \mathrm{H}$ NMR (400 MHz, Chloroform- $d$ ) $\delta 7.31-7.27(\mathrm{~m}, 1 \mathrm{H}), 7.21-7.14(\mathrm{~m}, 4 \mathrm{H})$, $4.00-3.86(\mathrm{~m}, 2 \mathrm{H}), 3.35(\mathrm{td}, J=11.8,2.1 \mathrm{~Hz}, 2 \mathrm{H}), 2.65-2.56(\mathrm{~m}, 2 \mathrm{H}), 1.61(\mathrm{~d}, J=7.5 \mathrm{~Hz}, 4 \mathrm{H})$, $1.52-1.39(\mathrm{~m}, 1 \mathrm{H}), 1.34(\mathrm{ddd}, J=10.9,8.1,5.8 \mathrm{~Hz}, 2 \mathrm{H}), 1.30-1.18(\mathrm{~m}, 4 \mathrm{H}) ;{ }^{13} \mathrm{C}$ NMR $(101$ MHz, Chloroform- $d$ ) $\delta$ 142.5, 128.2, 128.1, 125.4, 68.0, 36.6, 35.8, 34.7, 33.0, 31.5, 25.8; FTIR (neat) 2926, 2852, 1094, $698 \mathrm{~cm}^{-1}$; HRMS $(\mathrm{ESI}+)[\mathrm{M}+\mathrm{H}]^{+}$calculated for $\mathrm{C}_{15} \mathrm{H}_{23} \mathrm{O}: 219.1747$, found 219.1736 . 


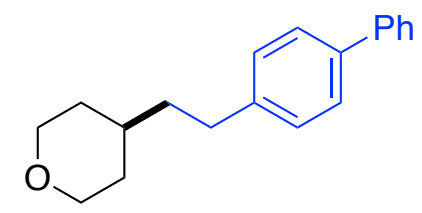

4-(2-\{[1,1'-Biphenyl]-4-yl $\}$ ethyl)oxane (15). Prepared via General Procedure A using pyridinium salt 3a. During work-up, the oxidation step with $\mathrm{H}_{2} \mathrm{O}_{2}$ was used. The crude mixture was purified by silica gel chromatography $\left(10 \% \rightarrow 50 \% \mathrm{Et}_{2} \mathrm{O} /\right.$ hexanes) to give $\mathbf{1 5}$ (run 1: $135 \mathrm{mg}, 51 \%$; run 2: $142 \mathrm{mg}, 54 \%)$ as a yellow oil: ${ }^{1} \mathrm{H}$ NMR (600 MHz, Chloroform- $d$ ) $\delta 7.60-7.56(\mathrm{~m}, 2 \mathrm{H}), 7.54-$ $7.50(\mathrm{~m}, 2 \mathrm{H}), 7.43(\mathrm{t}, J=7.7 \mathrm{~Hz}, 2 \mathrm{H}), 7.33(\mathrm{td}, J=7.2,1.3 \mathrm{~Hz}, 1 \mathrm{H}), 7.25(\mathrm{~s}, 2 \mathrm{H}), 3.97$ (ddd, $J=$ 12.4, 4.9, $1.7 \mathrm{~Hz}, 2 \mathrm{H}), 3.38(\mathrm{td}, J=11.8,2.0 \mathrm{~Hz}, 2 \mathrm{H}), 2.71-2.65(\mathrm{~m}, 2 \mathrm{H}), 1.68(\mathrm{dt}, J=13.2,2.3$ $\mathrm{Hz}, 2 \mathrm{H}), 1.65-1.59$ (m, 2H), $1.40-1.29$ (m, 3H); ${ }^{13} \mathrm{C}$ NMR (101 MHz, Chloroform-d) $\delta 141.8$, 141.2, 138.8, 128.75, 128.73, 127.10, 127.03, 127.0, 68.2 , 38.9, 34.7, 33.2, 32.4; FTIR (neat) 2917, 2837, 1094, $767 \mathrm{~cm}^{-1}$; HRMS (ESI+) $[\mathrm{M}+\mathrm{H}]^{+}$calculated for $\mathrm{C}_{19} \mathrm{H}_{23} \mathrm{O}: 267.1749$, found 267.1735.

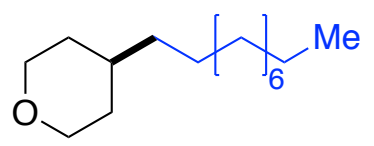

4-Decyloxane (16). Prepared via General Procedure A using pyridinium salt 3a. During work-up, the oxidation step with $\mathrm{H}_{2} \mathrm{O}_{2}$ was used. The crude mixture was purified by silica gel chromatography (100\% toluene) to give 16 (run 1: $96 \mathrm{mg}, 43 \%$; run 2: $112 \mathrm{mg}, 50 \%$ ) as a light yellow oil: ${ }^{1} \mathrm{H}$ NMR (600 MHz, Chloroform-d) $\delta 3.94(\mathrm{dtd}, J=11.6,2.4,1.1 \mathrm{~Hz}, 2 \mathrm{H}), 3.36(\mathrm{td}, J$ $=11.8,2.1 \mathrm{~Hz}, 2 \mathrm{H}), 1.61-1.54(\mathrm{~m}, 2 \mathrm{H}), 1.44(\mathrm{~m}, 1 \mathrm{H}), 1.31-1.21(\mathrm{~m}, 20 \mathrm{H}), 0.88(\mathrm{t}, J=7.0 \mathrm{~Hz}$, $3 \mathrm{H}),{ }^{13} \mathrm{C}$ NMR (101 MHz, Chloroform-d) $\delta$ 68.4, 37.1, 35.1, 33.4, 32.1, 31.1, 30.0, 29.82, 29.79 29.5, 26.5, 22.8, 14.3; FTIR (neat) 2954, 2923, 2852, $1465 \mathrm{~cm}^{-1}$; HRMS (ESI+) $[\mathrm{M}+\mathrm{H}]^{+}$calculated for $\mathrm{C}_{15} \mathrm{H}_{31} \mathrm{O}: 227.2375$, found 227.2368.

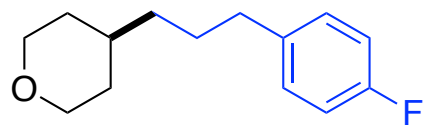

4-[3-(4-Fluorophenyl)propyl]oxane (17). Prepared via General Procedure A using pyridinium salt 3a. During work-up, the oxidation step with $\mathrm{H}_{2} \mathrm{O}_{2}$ was used. The crude mixture was purified by silica gel chromatography $\left(5 \% \rightarrow 10 \% \mathrm{Et}_{2} \mathrm{O} /\right.$ hexanes) to give 17 (run $1: 123 \mathrm{mg}, 56 \%$; run 2: $112 \mathrm{mg}, 52 \%$ ) as a light yellow oil: ${ }^{1} \mathrm{H}$ NMR (400 MHz, Chloroform- $d$ ) $\delta 7.17-7.09$ (m, 2H), $7.03-6.91(\mathrm{~m}, 2 \mathrm{H}), 3.94(\mathrm{dd}, J=11.5,4.5 \mathrm{~Hz}, 2 \mathrm{H}), 3.35(\mathrm{td}, J=11.8,2.1 \mathrm{~Hz}, 2 \mathrm{H}), 2.56(\mathrm{t}, J=$ $7.7 \mathrm{~Hz}, 2 \mathrm{H}), 1.64-1.58(\mathrm{~m}, 3 \mathrm{H}), 1.47(\mathrm{ddt}, J=10.9,7.6,3.7 \mathrm{~Hz}, 1 \mathrm{H}), 1.31-1.23(\mathrm{~m}, 5 \mathrm{H}) ;{ }^{13} \mathrm{C}$ 
NMR (101 MHz, Chloroform- $d) \delta 161.3\left(\mathrm{~d}, J_{\mathrm{C}-\mathrm{F}}=243.4 \mathrm{~Hz}\right) 138.3\left(\mathrm{~d}, J_{\mathrm{C}-\mathrm{F}}=3.0 \mathrm{~Hz}\right), 129.8\left(\mathrm{~d}, J_{\mathrm{C}-}\right.$ $\mathrm{F}=8.1 \mathrm{~Hz}), 115.12\left(\mathrm{~d}, J_{\mathrm{C}-\mathrm{F}}=21.2 \mathrm{~Hz}\right), 68.3,36.6,35.4,35.0,33.3,28.6 ;{ }^{19} \mathrm{~F} \mathrm{NMR}(376 \mathrm{MHz}$, Chloroform- $d$ ) $\delta-118.03$; FTIR (neat) 2926, 2854, 1265, $737 \mathrm{~cm}^{-1}$; HRMS (ESI+) $[\mathrm{M}+\mathrm{H}]^{+}$ calculated for $\mathrm{C}_{14} \mathrm{H}_{20} \mathrm{FO}$ : 223.1498, found 223.1400.

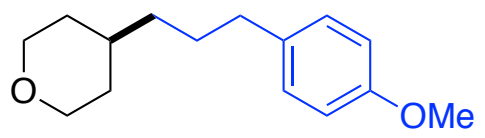

4-[3-(4-Methoxyphenyl)propyl]oxane (18). Prepared via General Procedure A using pyridinium salt 3a. During work-up, the oxidation step with $\mathrm{H}_{2} \mathrm{O}_{2}$ was used. The crude mixture was purified by silica gel chromatography $\left(2 \% \rightarrow 7 \% \rightarrow 15 \% \mathrm{Et}_{2} \mathrm{O} /\right.$ hexanes) to give $\mathbf{1 8}$ (run 1: $185 \mathrm{mg}, 79 \%$; run 2: $187 \mathrm{mg}, 77 \%$ ) as a light yellow oil: ${ }^{1} \mathrm{H}$ NMR $(400 \mathrm{MHz}$, Chloroform- $d$ ) $\delta 7.16-7.03$ (m, 2H), $6.88-6.77(\mathrm{~m}, 2 \mathrm{H}), 3.94(\mathrm{ddt}, J=11.4,4.4,1.1 \mathrm{~Hz}, 2 \mathrm{H}), 3.79$ (s, 3H), 3.36 (td, $J=11.8,2.1$ $\mathrm{Hz}, 2 \mathrm{H}), 2.54(\mathrm{t}, J=7.7 \mathrm{~Hz}, 2 \mathrm{H}), 1.66-1.55(\mathrm{~m}, 4 \mathrm{H}), 1.55-1.43(\mathrm{~m}, 1 \mathrm{H}), 1.33-1.20(\mathrm{~m}, 4 \mathrm{H}),{ }^{13} \mathrm{C}$ NMR (101 MHz, Chloroform-d) $\delta$ 157.8, 134.8, 129.3, 113.8, 68.3, 55.4, 36.7, 35.3, 35.1, 33.3, 28.7; FTIR (neat) 2927, 2837, 1512, 1245, $1036 \mathrm{~cm}^{-1}$; HRMS (ESI+) $[\mathrm{M}+\mathrm{H}]^{+}$calculated for $\mathrm{C}_{15} \mathrm{H}_{23} \mathrm{O}_{2}: 235.1698$, found 235.1685 .

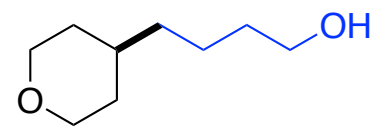

4-(Tetrahydro-2H-pyran-4-yl)-1-butanol (19). Prepared via General Procedure A using pyridinium salt 3a. During work-up, the oxidation step with $\mathrm{H}_{2} \mathrm{O}_{2}$ was not used. The crude mixture was purified by silica gel chromatography $\left(5 \% \rightarrow 50 \% \mathrm{Et}_{2} \mathrm{O} /\right.$ hexanes) to give 19 (run 1: $158 \mathrm{mg}$, 68\%; run 2: $159 \mathrm{mg}, 69 \%$ ) as a yellow oil: ${ }^{1} \mathrm{H}$ NMR (600 MHz, Chloroform-d) $\delta 3.97-3.91$ (m, $2 \mathrm{H}), 3.65(\mathrm{t}, J=6.6 \mathrm{~Hz}, 2 \mathrm{H}), 3.36(\mathrm{td}, J=11.8,2.1 \mathrm{~Hz}, 2 \mathrm{H}), 1.62-1.58(\mathrm{~m}, 2 \mathrm{H}), 1.57-1.53(\mathrm{~m}$, 2H), $1.47(\mathrm{dtd}, J=14.4,7.5,6.9,3.6 \mathrm{~Hz}, 1 \mathrm{H}), 1.41-1.35(\mathrm{~m}, 2 \mathrm{H}), 1.30-1.24(\mathrm{~m}, 4 \mathrm{H})$; ${ }^{13} \mathrm{C}$ NMR (101 MHz, Chloroform- $d$ ) $\delta$ 68.3, 63.1, 36.9, 35.1, 33.3, 33.0, 22.7; FTIR (neat) 3344, 2922, 2849, 1095, $736 \mathrm{~cm}^{-1}$; HRMS (ESI+) $[\mathrm{M}+\mathrm{H}]^{+}$calculated for $\mathrm{C}_{9} \mathrm{H}_{19} \mathrm{O}_{2}: 159.1385$, found 159.1374 .

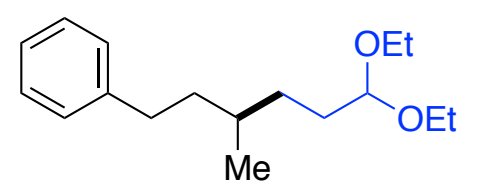

(6,6-Diethoxy-3-methylhexyl)benzene (20). Prepared via General Procedure A using pyridinium salt 3i. During work-up, the oxidation step with $\mathrm{H}_{2} \mathrm{O}_{2}$ was used. The crude mixture was purified 
by silica gel chromatography $\left(1 \% \rightarrow 5 \% \mathrm{Et}_{2} \mathrm{O} /\right.$ hexanes) to give 20 (run $1: 175 \mathrm{mg}, 65 \%$; run 2 : $188 \mathrm{mg}, 70 \%)$ as a yellow oil: ${ }^{1} \mathrm{H}$ NMR (400 MHz, Chloroform- $\left.d\right) \delta 7.27(\mathrm{~m}, 2 \mathrm{H}), 7.18(\mathrm{~m}, 3 \mathrm{H})$, $4.46(\mathrm{t}, J=5.7 \mathrm{~Hz}, 1 \mathrm{H}), 3.64(\mathrm{dq}, J=9.4,7.1 \mathrm{~Hz}, 2 \mathrm{H}), 3.49$ (dq, $J=9.4,7.1 \mathrm{~Hz}, 2 \mathrm{H}), 2.74-2.49$ $(\mathrm{m}, 2 \mathrm{H}), 1.73-1.36(\mathrm{~m}, 7 \mathrm{H}), 1.21(\mathrm{t}, J=7.0 \mathrm{~Hz}, 6 \mathrm{H}), 0.94(\mathrm{~d}, J=6.0 \mathrm{~Hz}, 3 \mathrm{H}) ;{ }^{13} \mathrm{C}$ NMR $(101$ MHz, Chloroform- $d$ ) $\delta$ 143.1, 128.5, 128.4, 125.7, 103.3, 62.0, 60.9, 39.0, 33.6, 32.5, 31.7, 31.1, 19.7, 15.5; FTIR (neat) 2973, 2928, 1456, 1127, 1063, $698 \mathrm{~cm}^{-1}$; HRMS (ESI+) [M-OEt] ${ }^{+}$ calculated for $\mathrm{C}_{15} \mathrm{H}_{23} \mathrm{O}: 219.1749$, found 219.1743 .

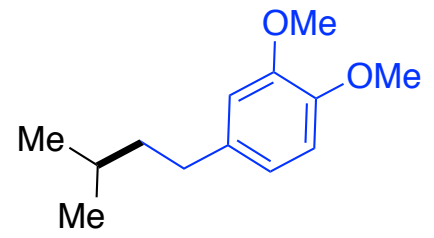

1,2-Dimethoxy-4-(3-methylbutyl)benzene (21). Prepared via General Procedure A using pyridinium salt 3j. During work-up, the oxidation step with $\mathrm{H}_{2} \mathrm{O}_{2}$ was used. The crude mixture was purified by silica gel chromatography $(2 \% \rightarrow 10 \%$ EtOAc/hexanes) to give 21 (run 1: $107 \mathrm{mg}$, 51\%; run 2: $107 \mathrm{mg}, 51 \%$ ) as a light yellow oil: ${ }^{1} \mathrm{H}$ NMR (400 MHz, Chloroform- $d$ ) $\delta 6.81-6.77$ $(\mathrm{m}, 1 \mathrm{H}), 6.72(\mathrm{~d}, J=7.2 \mathrm{~Hz}, 2 \mathrm{H}), 3.88(\mathrm{~s}, 3 \mathrm{H}), 3.86(\mathrm{~s}, 3 \mathrm{H}), 2.61-2.51(\mathrm{~m}, 2 \mathrm{H}), 1.62-1.55(\mathrm{~m}$, $1 \mathrm{H}), 1.50-1.41(\mathrm{~m}, 2 \mathrm{H}), 0.93(\mathrm{~d}, J=6.5 \mathrm{~Hz}, 6 \mathrm{H}) ;{ }^{13} \mathrm{C}$ NMR (101 MHz, Chloroform- $d$ ) $\delta 148.8$, 147.0, 135.9, 120.2 , 111.7, 111.2, 56.0, 55.9, 41.2, 33.6, 27.9, 22.7; FTIR (neat) 2952, 1515, 1261, $1030 \mathrm{~cm}^{-1}$; HRMS (ESI+) $[\mathrm{M}+\mathrm{H}]^{+}$calculated for $\mathrm{C}_{13} \mathrm{H}_{21} \mathrm{O}_{2}: 209.1542$, found 209.1530 .

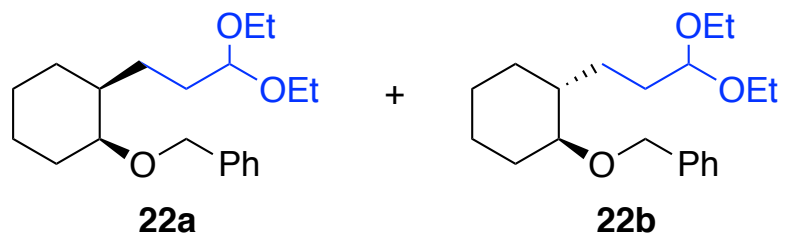

(\{[(1S)-2-(3,3-Diethoxypropyl)cyclohexyl]oxy\}methyl)benzene (22). Prepared via General Procedure A using pyridinium salt 3k. During work-up, the oxidation step with $\mathrm{H}_{2} \mathrm{O}_{2}$ was used. The crude mixture was purified by silica gel chromatography $\left(10 \% \mathrm{Et}_{2} \mathrm{O} /\right.$ hexanes) to give $22 \mathrm{a}$ (run 1: $100 \mathrm{mg}, 32 \%$; run 2: $111 \mathrm{mg}, 35 \%$ ) and 22b (run 1: $100 \mathrm{mg}$, 32\%; run 2: $111 \mathrm{mg}, 35 \%$ ), both as light yellow oils. The combined yield was $66 \%$ (run 1: $200 \mathrm{mg}, 63 \%$; run 2: $222 \mathrm{mg}, 69 \%$ ) as a 1:1 mixture of diastereomers. 
22a: ${ }^{1} \mathrm{H}$ NMR (600 MHz, Chloroform- $\left.d\right) \delta 7.33-7.18(\mathrm{~m}, 5 \mathrm{H}), 4.52(\mathrm{~d}, J=11.9 \mathrm{~Hz}, 1 \mathrm{H})$, $4.37(\mathrm{t}, J=5.6 \mathrm{~Hz}, 1 \mathrm{H}), 4.31(\mathrm{~d}, J=11.9 \mathrm{~Hz}, 1 \mathrm{H}), 3.54(\mathrm{ddq}, J=9.3,8.4,7.0 \mathrm{~Hz}, 2 \mathrm{H}), 3.47$ (dt, $J$ $=5.1,2.4 \mathrm{~Hz}, 1 \mathrm{H}), 3.44-3.33(\mathrm{~m}, 2 \mathrm{H}), 1.89(\mathrm{dt}, J=14.5,4.5 \mathrm{~Hz}, 1 \mathrm{H}), 1.59-1.42(\mathrm{~m}, 6 \mathrm{H}), 1.37$ - $1.17(\mathrm{~m}, 6 \mathrm{H}), 1.12$ (q, $J=7.2 \mathrm{~Hz}, 6 \mathrm{H}) .{ }^{13} \mathrm{C}$ NMR (101 MHz, Chloroform- $d$ ) $\delta 139.5,128.2$, $127.4,127.2$, 103.3, 70.1, 60.9, 60.7, 40.8, 31.3, 31.0, 28.5, 27.5, 25.0, 21.1, 15.4; FTIR (neat) 2928, 1443, 1061, $696 \mathrm{~cm}^{-1}$; HRMS (LIFDI+) $[\mathrm{M}-\mathrm{H}]^{+}$calculated for $\mathrm{C}_{20} \mathrm{H}_{31} \mathrm{O}_{3}: 319.2273$, found 319.2281.

22b: ${ }^{1} \mathrm{H}$ NMR (400 MHz, Chloroform- $d$ ) $\delta 7.38-7.27(\mathrm{~m}, 5 \mathrm{H}), 4.63(\mathrm{~d}, J=11.5 \mathrm{~Hz}, 1 \mathrm{H})$, $4.46(\mathrm{t}, J=5.8 \mathrm{~Hz}, 1 \mathrm{H}), 4.42(\mathrm{~d}, J=11.5 \mathrm{~Hz}, 1 \mathrm{H}), 3.62(\mathrm{dqd}, J=9.3,7.1,2.2 \mathrm{~Hz}, 2 \mathrm{H}), 3.47(\mathrm{dq}$, $J=9.3,7.0 \mathrm{~Hz}, 2 \mathrm{H}), 3.01(\mathrm{td}, J=9.5,4.3 \mathrm{~Hz}, 1 \mathrm{H}), 2.19-2.10(\mathrm{~m}, 1 \mathrm{H}), 1.91-1.78(\mathrm{~m}, 2 \mathrm{H}), 1.78$ - $1.73(\mathrm{~m}, 1 \mathrm{H}), 1.73-1.49(\mathrm{~m}, 5 \mathrm{H}), 1.45-1.35(\mathrm{~m}, 1 \mathrm{H}), 1.19(\mathrm{~m}, 8 \mathrm{H}), 1.01-0.91(\mathrm{~m}, 1 \mathrm{H}) ;{ }^{13} \mathrm{C}$ NMR (101 MHz, Chloroform-d) $\delta 139.1,128.3,127.8,127.4,103.4,81.7,70.6,60.8,60.7$, 42.9, 31.1, 30.6, 30.3, 27.2, 25.4, 24.7, 15.4, 15.4; FTIR (neat) 2972, 2926, 2855, 1453, 1069, $697 \mathrm{~cm}^{-}$ 1; HRMS (ESI+) $[\mathrm{M}-\mathrm{Et}+\mathrm{H}]^{+}$calculated for $\mathrm{C}_{18} \mathrm{H}_{27} \mathrm{O}_{3}: 291.1960$, found 291.1946.

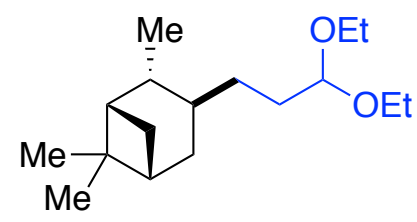

1R,3S,5R)-2-(3,3-Diethoxypropyl)-3,6,6-trimethylbicyclo[3.1.1]heptane (23). Prepared via General Procedure A using pyridinium salt 31. During work-up, the oxidation step with $\mathrm{H}_{2} \mathrm{O}_{2}$ was used. The crude mixture was purified by silica gel chromatography ( $100 \%$ toluene) to give $\mathbf{2 3}$ (run 1: $275 \mathrm{mg}, 54 \%$; run 2: $269 \mathrm{mg}, 50 \%$ ) as a single diastereomer and a light yellow oil: ${ }^{1} \mathrm{H}$ NMR $(600 \mathrm{MHz}$, Chloroform- $d) \delta 4.48(\mathrm{t}, J=5.6 \mathrm{~Hz}, 1 \mathrm{H}), 3.65(\mathrm{dqd}, J=9.2,7.0,2.0 \mathrm{~Hz}, 2 \mathrm{H}), 3.58-$ $3.46(\mathrm{~m}, 2 \mathrm{H}), 2.27$ (dtt, $J=12.1,8.0,3.9 \mathrm{~Hz}, 1 \mathrm{H}), 2.13(\mathrm{dddd}, J=11.3,9.4,4.3,2.4 \mathrm{~Hz}, 1 \mathrm{H}), 1.89$ $(\mathrm{tt}, J=6.0,3.0 \mathrm{~Hz}, 1 \mathrm{H}), 1.77-1.67(\mathrm{~m}, 2 \mathrm{H}), 1.67-1.51(\mathrm{~m}, 5 \mathrm{H}), 1.46-1.38(\mathrm{~m}, 1 \mathrm{H}), 1.21(\mathrm{t}, J$ $=7.0 \mathrm{~Hz}, 6 \mathrm{H}), 1.18(\mathrm{~s}, 3 \mathrm{H}), 1.01(\mathrm{~d}, J=6.9 \mathrm{~Hz}, 3 \mathrm{H}), 0.99(\mathrm{~s}, 3 \mathrm{H}), 0.75(\mathrm{~d}, J=9.5 \mathrm{~Hz}, 1 \mathrm{H}) ;{ }^{13} \mathrm{C}$ NMR (101 MHz, Chloroform- $d$ ) $\delta$ 103.3, 61.0, 60.8, 48.3, 43.9, 42.1, 38.9, 36.4, 36.1, 34.8, 34.2, 31.9, 28.2, 23.1, 21.9, 15.5, 14.3. FTIR (neat) 2923, 2855, 1727, 1067, $697 \mathrm{~cm}^{-1}$; HRMS (ESI+) $[\mathrm{M}-\mathrm{OEt}]^{+}$calculated for $\mathrm{C}_{15} \mathrm{H}_{27} \mathrm{O}: 223.2062$, found 223.2049 . 


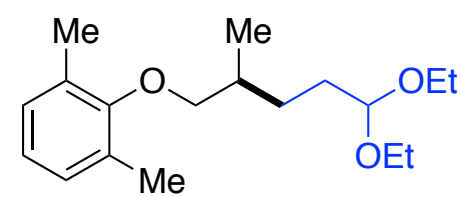

2-[(5,5-Diethoxy-2-methylpentyl)oxy]-1,3-dimethylbenzene (24). Prepared via General Procedure A using pyridinium salt 3m. During work-up, the oxidation step with $\mathrm{H}_{2} \mathrm{O}_{2}$ was used. The crude mixture was purified by silica gel chromatography $\left(5 \% \rightarrow 30 \% \mathrm{Et}_{2} \mathrm{O} /\right.$ hexanes $)$ to give 24 (run 1: $122 \mathrm{mg}, 41 \%$; run 2: $128 \mathrm{mg}, 43 \%$ ) as a yellow oil: ${ }^{1} \mathrm{H}$ NMR (400 MHz, Chloroformd) $\delta 7.00(\mathrm{~d}, J=7.4 \mathrm{~Hz}, 2 \mathrm{H}), 6.90(\mathrm{~m}, 6.8 \mathrm{~Hz}, 1 \mathrm{H}), 4.51(\mathrm{t}, J=5.6 \mathrm{~Hz}, 1 \mathrm{H}), 3.71-3.58(\mathrm{~m}, 3 \mathrm{H})$, $3.52(\mathrm{~m}, 3 \mathrm{H}), 2.27(\mathrm{~s}, 6 \mathrm{H}), 1.96(\mathrm{dp}, J=13.0,6.4 \mathrm{~Hz}, 1 \mathrm{H}), 1.82-1.58(\mathrm{~m}, 3 \mathrm{H}), 1.39-1.27(\mathrm{~m}$, $1 \mathrm{H}), 1.21(\mathrm{~d}, J=7.0, \mathrm{~Hz}, 6 \mathrm{H}), 1.10(\mathrm{~d}, J=6.7 \mathrm{~Hz}, 3 \mathrm{H}) ;{ }^{13} \mathrm{C}$ NMR $(101 \mathrm{MHz}$, Chloroform- $d) \delta$ 156.0, 131.1, 128.9, 123.8, 103.3, 61.8, 61.1, 34.4, 31.4, 28.6, 17.2, 16.5, 15.5; FTIR (neat) 2973, 2927, 1473, 1203, 1062, $769 \mathrm{~cm}^{-1}$; HRMS (LIFDI) [M] calculated for $\mathrm{C}_{18} \mathrm{H}_{30} \mathrm{O}_{3}: 294.2195$, found 294.2181.

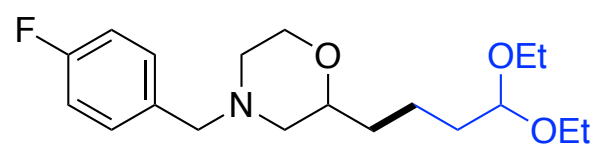

2-(4,4-Diethoxybutyl)-4-[(4-fluorophenyl)methyl]morpholine (25). Prepared via General Procedure A using pyridinium salt 3n. During work-up, the oxidation step with $\mathrm{H}_{2} \mathrm{O}_{2}$ was not used. The crude mixture was purified by silica gel chromatography $\left(20 \% \rightarrow 80 \% \mathrm{Et}_{2} \mathrm{O} /\right.$ hexanes $)$ to give 25 (run 1: $239 \mathrm{mg}, 69 \%$; run 2: $225 \mathrm{mg}, 64 \%$ ) as a yellow oil: ${ }^{1} \mathrm{H}$ NMR (400 MHz, Chloroformd) $\delta 7.31-7.26(\mathrm{~m}, 2 \mathrm{H}), 7.04-6.96(\mathrm{~m}, 2 \mathrm{H}), 4.46(\mathrm{t}, J=5.7 \mathrm{~Hz}, 1 \mathrm{H}), 3.83(\mathrm{ddd}, J=11.4,3.4$, $1.5 \mathrm{~Hz}, 1 \mathrm{H}), 3.62$ (dddd, $J=14.2,12.3,5.9,3.6 \mathrm{~Hz}, 3 \mathrm{H}), 3.51-3.41(\mathrm{~m}, 5 \mathrm{H}), 2.73-2.58(\mathrm{~m}, 2 \mathrm{H})$, $2.12(\mathrm{td}, J=11.4,3.4 \mathrm{~Hz}, 1 \mathrm{H}), 1.82(\mathrm{t}, J=10.6 \mathrm{~Hz}, 1 \mathrm{H}), 1.63-1.56(\mathrm{~m}, 2 \mathrm{H}), 1.54-1.33(\mathrm{~m}, 4 \mathrm{H})$, $1.19(\mathrm{td}, J=7.1,1.2 \mathrm{~Hz}, 6 \mathrm{H}) ;{ }^{13} \mathrm{C}$ NMR $\left(101 \mathrm{MHz}\right.$, Chloroform-d) $\delta 162.2\left(\mathrm{~d}, J_{\mathrm{C}-\mathrm{F}}=246.4 \mathrm{~Hz}\right)$, $133.6\left(\mathrm{~d}, J_{\mathrm{C}-\mathrm{F}}=3.0 \mathrm{~Hz}\right), 130.8\left(\mathrm{~d}, J_{\mathrm{C}-\mathrm{F}}=8.9 \mathrm{~Hz}\right), 128.5\left(\mathrm{~d}, J_{\mathrm{C}-\mathrm{F}}=11.1 \mathrm{~Hz}\right), 115.2\left(\mathrm{~d}, J_{\mathrm{C}-\mathrm{F}}=21.2\right.$ $\mathrm{Hz}), 102.9,75.7,66.9,62.6,61.2,61.0,58.7,53.2,33.7,33.6,20.8,15.5 ;{ }^{19} \mathrm{~F}$ NMR (565 MHz, Chloroform- $d$ ) $\delta-115.85$; FTIR (neat) 2973, 2929, 2869, 1113, 1062, $850 \mathrm{~cm}^{-1}$; HRMS (ESI+) $[\mathrm{M}+\mathrm{H}]^{+}$calculated for $\mathrm{C}_{19} \mathrm{H}_{31} \mathrm{FNO}_{3}: 340.2288$, found 340.2271. 


\section{General Procedure B: Vinylation of Alkyl Pyridinium Salts with Alkynes}
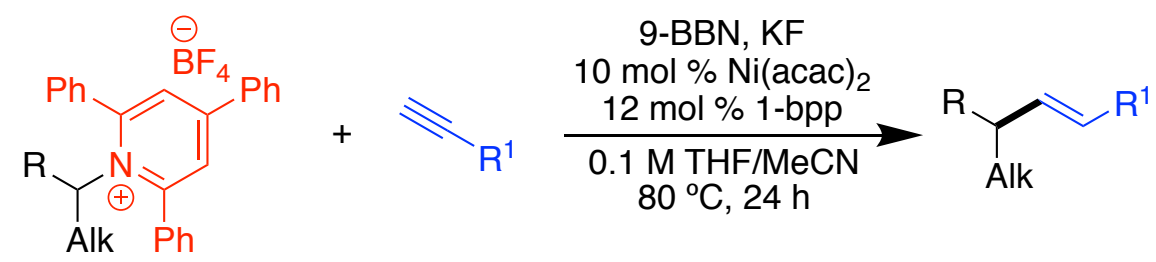

Vinylation of alkyl pyridinium salts was accomplished using a very similar procedure to General Procedure A, except that the alkene (General Procedure A) was replaced by an alkyne.

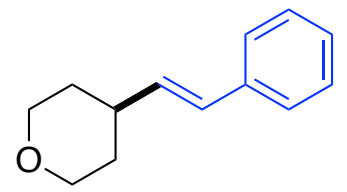

(E)-4-Styryltetrahydro-2H-pyran (26). Prepared via General Procedure B using pyridinium salt 3a. During work-up, the oxidation step with $\mathrm{H}_{2} \mathrm{O}_{2}$ was used. The crude mixture was purified by silica gel chromatography ( $10 \% \mathrm{Et}_{2} \mathrm{O} /$ hexanes) to give $\mathbf{2 6}$ (run 1:113 $\mathrm{mg}, 60 \%$, run 2:118 $\mathrm{mg}, 63 \%$ ) as a light yellow oil: ${ }^{1} \mathrm{H}$ NMR (400 MHz, Chloroform- $d$ ) $\delta 7.36(\mathrm{~d}, J=7.2 \mathrm{~Hz}, 2 \mathrm{H}), 7.30(\mathrm{t}, J=$ $7.6 \mathrm{~Hz}, 2 \mathrm{H}), 7.21$ (t, $J=7.2 \mathrm{~Hz}, 1 \mathrm{H}), 6.39$ (d, $J=16.0 \mathrm{~Hz}, 1 \mathrm{H}), 6.16$ (dd, $J=16.0,6.8 \mathrm{~Hz}, 1 \mathrm{H}$ ), 4.01 (ddd, $J=11.3,4.8,1.8 \mathrm{~Hz}, 2 \mathrm{H}), 3.47$ (td, $J=11.6,2.2 \mathrm{~Hz}, 2 \mathrm{H}$ ), 2.38 (ddd, $J=11.2,8.9,5.0$ $\mathrm{Hz}, 1 \mathrm{H}), 1.74-1.67(\mathrm{~m}, 2 \mathrm{H}), 1.63-1.51(\mathrm{~m}, 2 \mathrm{H}) ;{ }^{13} \mathrm{C}$ NMR (101 MHz, Chloroform- $d$ ) $\delta$ 137.6, $134.7,128.7,128.4,127.2,126.2,67.9,38.5,32.8$. The spectral data matches that previously reported in the literature. ${ }^{3}$

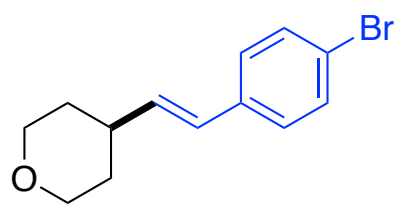

(E)-4-(4-Bromostyryl)tetrahydro-2H-pyran (27). Prepared via General Procedure B using pyridinium salt 3a, except that $\mathrm{KF}, 9-\mathrm{BBN}$, and alkyne were stirred at $80^{\circ} \mathrm{C}$ for $1 \mathrm{~h}$ instead of 30 min prior to addition of the other reagents. During work-up, the oxidation step with $\mathrm{H}_{2} \mathrm{O}_{2}$ was used. The crude mixture was purified by silica gel chromatography $\left(5 \% \rightarrow 10 \% \mathrm{Et}_{2} \mathrm{O} /\right.$ hexanes) to give $27(127 \mathrm{mg}, 48 \%)$ as a yellow solid (mp 56-63 $\left.{ }^{\circ} \mathrm{C}\right):{ }^{1} \mathrm{H}$ NMR $(600 \mathrm{MHz}$, Chloroform- $d$ ) $\delta$ $7.45-7.38$ (m, 2H), $7.24-7.19$ (m, 2H), $6.35-6.29$ (m, 1H), 6.15 (dd, $J=16.0,6.8 \mathrm{~Hz}, 1 \mathrm{H})$, 4.01 (ddd, $J=11.7,4.6,1.9 \mathrm{~Hz}, 2 \mathrm{H}$ ), 3.46 (td, $J=11.8,2.2 \mathrm{~Hz}, 2 \mathrm{H}$ ), 2.37 (ddt, $J=10.9,6.9,3.3$ $\mathrm{Hz}, 1 \mathrm{H}), 1.72-1.64(\mathrm{~m}, 2 \mathrm{H}), 1.61-1.53(\mathrm{~m}, 2 \mathrm{H}) ;{ }^{13} \mathrm{C}$ NMR (151 MHz, Chloroform- $d$ ) $\delta 136.7$, 
$135.6,131.75,127.8,127.4,120.9,67.8,38.5,32.7$. The spectral data matches that previously reported in the literature. ${ }^{4}$

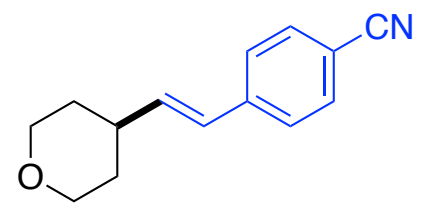

(E)-4-(2-(Tetrahydro-2H-pyran-4-yl)vinyl)benzonitrile (30). Prepared via General Procedure B using pyridinium salt 3a. During work-up, the oxidation step with $\mathrm{H}_{2} \mathrm{O}_{2}$ was not used. The crude mixture was purified by silica gel chromatography $\left(10 \% \rightarrow 15 \% \mathrm{Et}_{2} \mathrm{O} /\right.$ hexanes) to give 30 (164 mg, 71\%) as a light yellow oil: ${ }^{1} \mathrm{H}$ NMR (400 MHz, Chloroform- $\left.d\right) \delta 7.66-7.54(\mathrm{~m}, 2 \mathrm{H}), 7.49-$ $7.37(\mathrm{~m}, 2 \mathrm{H}), 6.39(\mathrm{~d}, J=16.1 \mathrm{~Hz}, 1 \mathrm{H}), 6.30(\mathrm{dd}, J=16.0,6.5 \mathrm{~Hz}, 1 \mathrm{H}), 4.02(\mathrm{ddd}, J=11.6,4.5$, $1.9 \mathrm{~Hz}, 2 \mathrm{H}), 3.47(\mathrm{td}, J=11.7,2.2 \mathrm{~Hz}, 2 \mathrm{H}), 2.42(\mathrm{dtt}, J=10.8,7.0,3.7 \mathrm{~Hz}, 1 \mathrm{H}), 1.71(\mathrm{ddd}, J=$ 13.2, 4.1, 2.1 Hz, 2H), 1.57 (dtd, $J=13.5,11.6,4.4 \mathrm{~Hz}, 2 \mathrm{H}) ;{ }^{13} \mathrm{C}$ NMR (101 MHz, Chloroform- $d$ ) $\delta 142.2,138.8,132.5,127.1,126.7,119.2,110.4,67.7,38.6,32.4$; FTIR (neat) 2871, 2361, 667-1; HRMS (ESI+) $[\mathrm{M}+\mathrm{H}]^{+}$calculated for $\mathrm{C}_{14} \mathrm{H}_{16} \mathrm{NO}: 214.1232$, found 214.1222.

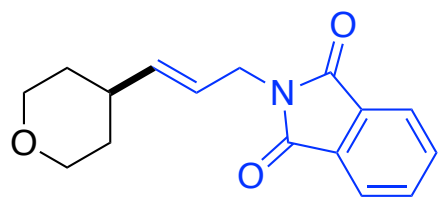

(E)-2-(3-(Tetrahydro-2H-pyran-4-yl)allyl)isoindoline-1,3-dione (29). Prepared via General Procedure B using pyridinium salt 3a, except that $\mathrm{KF}, 9-\mathrm{BBN}$, and alkyne were stirred at $80{ }^{\circ} \mathrm{C}$ for $1 \mathrm{~h}$ instead of $30 \mathrm{~min}$ prior to addition of the other reagents. During work-up, the oxidation step with $\mathrm{H}_{2} \mathrm{O}_{2}$ was used. The crude mixture was purified by silica gel chromatography (10\% $\mathrm{Et}_{2} \mathrm{O} /$ hexanes) to give 29 (106 mg, 53\%) as a light yellow solid (mp 60-65 $\left.{ }^{\circ} \mathrm{C}\right):{ }^{1} \mathrm{H}$ NMR (600 MHz, Chloroform- $d$ ) $\delta 7.85(\mathrm{dt}, J=6.4,3.2 \mathrm{~Hz}, 2 \mathrm{H}), 7.75-7.67$ (m, 2H), 5.70 (ddt, $J=15.5,6.3$, $1.4 \mathrm{~Hz}, 1 \mathrm{H}), 5.51(\mathrm{dtd}, J=15.4,6.2,1.4 \mathrm{~Hz}, 1 \mathrm{H}), 4.25(\mathrm{dt}, J=6.2,1.1 \mathrm{~Hz}, 2 \mathrm{H}), 3.93$ (ddd, $J=$ 11.8, 4.5, 2.0 Hz, 2H), 3.37 (td, $J=11.7,2.2 \mathrm{~Hz}, 2 \mathrm{H}), 2.23-2.13$ (m, 1H), 1.59 (ddd, $J=13.3$, 4.1, $2.0 \mathrm{~Hz}, 2 \mathrm{H}), 1.43$ (dtd, $J=13.4,11.6,4.4 \mathrm{~Hz}, 2 \mathrm{H}) ;{ }^{13} \mathrm{C}$ NMR (151 MHz, Chloroform-d) $\delta$ $168.1,138.9,134.1,132.3,123.4,121.9,67.8,39.7,37.6,32.4$; FTIR (neat) 2929, 2842, 1771, 1713, 1393, $720 \mathrm{~cm}^{-1}$; HRMS (ESI+) $[\mathrm{M}+\mathrm{H}]^{+}$calculated for $\mathrm{C}_{16} \mathrm{H}_{18} \mathrm{NO}_{3}$ : 272.1287, found 272.1275 . 


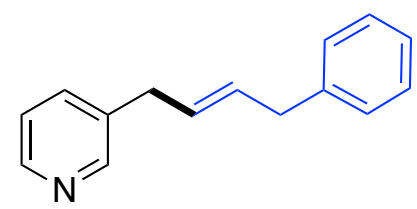

(E)-3-(4-Phenylbut-2-en-1-yl)pyridine (30). Prepared via General Procedure B using pyridinium salt 3o, except that KF, 9-BBN, and alkyne were stirred at $80{ }^{\circ} \mathrm{C}$ for $1 \mathrm{~h}$ instead of $30 \mathrm{~min}$ prior to addition of the other reagents. During work-up, the oxidation step with $\mathrm{H}_{2} \mathrm{O}_{2}$ was used. The crude mixture was purified by silica gel chromatography $\left(5 \% \rightarrow 10 \% \mathrm{Et}_{2} \mathrm{O} /\right.$ hexanes $)$ to give 30 (124 mg, $59 \%$ ) as a light yellow oil: ${ }^{1} \mathrm{H}$ NMR $(600 \mathrm{MHz}$, Chloroform- $d) \delta 8.48-8.43(\mathrm{~m}, 2 \mathrm{H}), 7.51(\mathrm{dt}, J$ $=7.9,1.8 \mathrm{~Hz}, 1 \mathrm{H}), 7.32-7.28(\mathrm{~m}, 2 \mathrm{H}), 7.25-7.20(\mathrm{~m}, 2 \mathrm{H}), 7.20-7.16(\mathrm{~m}, 2 \mathrm{H}), 5.76-5.60(\mathrm{~m}$, 2H), $3.38(\mathrm{t}, J=5.5 \mathrm{~Hz}, 4 \mathrm{H}) ;{ }^{13} \mathrm{C}$ NMR (151 MHz, Chloroform- $d$ ) $\delta 150.1,147.6,140.5,136.3$, $136.1,131.8,129.2,128.64,128.60,126.2,123.5,39.1,36.2$. The spectral data matches that previously reported in the literature..$^{5}$ 


\section{Preparation of Pyridinium Salts}

If previously reported, the pyridinium salts were prepared as previously described: $\mathbf{3 a}, \mathbf{3 c}, \mathbf{3 i}, \mathbf{3 k}$, $\mathbf{3 n} ;{ }^{6} \mathbf{3 e}, \mathbf{3 j} ;{ }^{7} \mathbf{3 f}-\mathbf{3 h}$ and $\mathbf{3 o} ;{ }^{8} \mathbf{3 b}$ and $\mathbf{3 n} ;{ }^{9} \mathbf{3 l} .{ }^{10}$
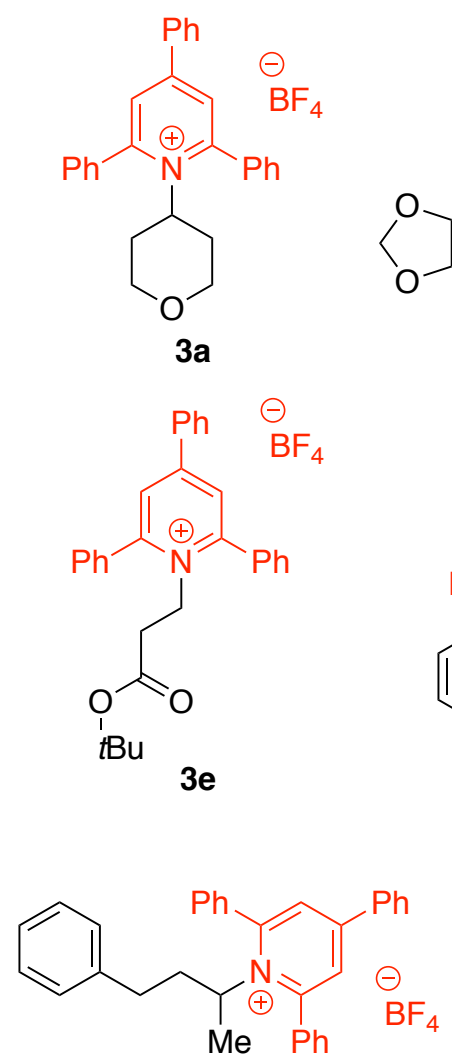

3i<smiles></smiles>

$3 m$<smiles></smiles>

$3 \mathbf{b}$<smiles></smiles>

$3 f$

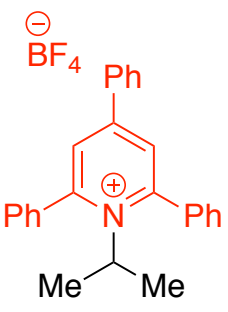

3j<smiles></smiles>

3c

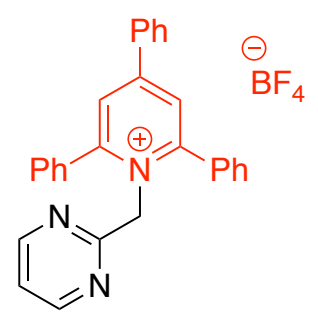

$3 g$<smiles>c1ccc(CO[C@@H]2CCCC[C@H]2[n+]2c(-c3ccccc3)cc(-c3ccccc3)cc2-c2ccccc2)cc1</smiles>

3k<smiles>[13CH2]c1ccccn1</smiles>

3d<smiles></smiles>

3h

3I<smiles>[13CH2]c1cc(-c2ccccc2)cc(-c2ccccc2)[n+]1Cc1cccnc1</smiles>

30 


\section{General Procedure C: Synthesis of Pyridinium Salts}
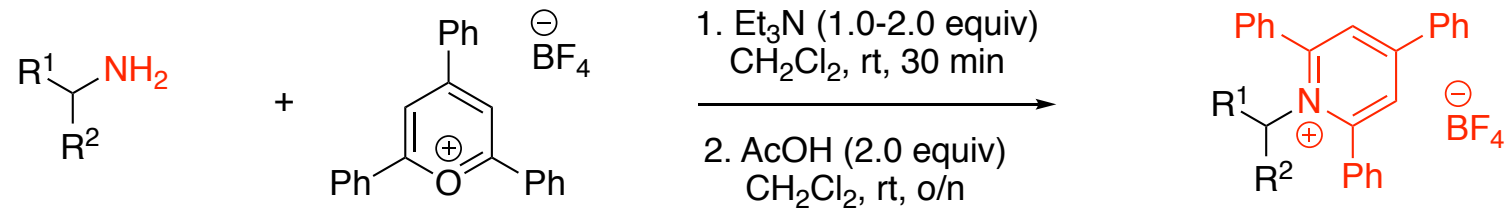

Under air, 2,4,6-triphenylpyrylium tetrafluoroborate (1.0 equiv), powdered activated $4 \AA \AA$ molecular sieves $(500 \mathrm{mg} / \mathrm{mmol})$, and $\mathrm{CH}_{2} \mathrm{Cl}_{2}$ were added to a round-bottomed flask was a stirbar. The alkyl amine (1.0 equiv) was added, and the flask was fitted with a septum. With a vent needle, $\mathrm{Et}_{3} \mathrm{~N}$ (1.0 equiv for free-base amines; 2.0 equiv for amine hydrochloride salts) was added via syringe. The vent needle was removed, and the mixture was stirred for $30 \mathrm{~min}$ at room temperature. The vent needle was re-inserted, and acetic acid (2.0 equiv) was added via syringe. The vent needle was removed, and the mixture was stirred overnight at room temperature. The mixture was filtered through a short Celite plug using $\mathrm{CH}_{2} \mathrm{Cl}_{2}$. The filtrate was then washed with aq. $\mathrm{HCl}(1.0 \mathrm{M}, 2 \mathrm{x}$ $30 \mathrm{~mL})$, sat. aq. $\mathrm{NaHCO}_{3}(2 \times 30 \mathrm{~mL})$, and sat. $\mathrm{NaCl}(2 \times 30 \mathrm{~mL})$, dried $\left(\mathrm{MgSO}_{4}\right)$, filtered, and concentrated. $\mathrm{Et}_{2} \mathrm{O}$ was added to the residue to precipitate the pyridinium salt. The solid pyridinium salt was then filtered and washed with $\mathrm{Et}_{2} \mathrm{O}$.

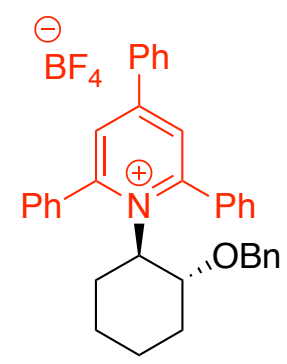

1-((Trans)-2-(benzyloxy)cyclohexyl)-2,4,6-triphenylpyridin-1-ium tetrafluoroborate (3k).

Prepared via General Procedure B from (1S,2S)-2-(benzyloxy)cyclohexan-1-amine HCl (2.42 g, $10.0 \mathrm{mmol}, 1.0$ equiv) and 2,4,6-triphenylpyrillium tetrafluoroborate (3.96 g, $10.0 \mathrm{mmol}, 1.0$ equiv) to give 3k $(5.06 \mathrm{~g}, 91 \%)$ as an orange solid $\left(\mathrm{mp} 145-148{ }^{\circ} \mathrm{C}\right):{ }^{1} \mathrm{H} \mathrm{NMR}(600 \mathrm{MHz}$, Chloroform- $d) \delta 8.13(\mathrm{~d}, J=6.1 \mathrm{~Hz}, 1 \mathrm{H}), 8.09-8.04(\mathrm{~m}, 1 \mathrm{H}), 7.92(\mathrm{~d}, J=2.4 \mathrm{~Hz}, 1 \mathrm{H}), 7.81-$ $7.78(\mathrm{~m}, 2 \mathrm{H}), 7.71(\mathrm{~d}, J=2.4 \mathrm{~Hz}, 1 \mathrm{H}), 7.65-7.51(\mathrm{~m}, 9 \mathrm{H}), 7.49-7.34(\mathrm{~m}, 4 \mathrm{H}), 7.04-7.00(\mathrm{~m}$, 2H), $6.94(\mathrm{~d}, J=7.7 \mathrm{~Hz}, 1 \mathrm{H}), 4.78(\mathrm{ddd}, J=13.1,10.3,3.1 \mathrm{~Hz}, 1 \mathrm{H}), 4.57(\mathrm{~d}, J=12.1 \mathrm{~Hz}, 1 \mathrm{H})$, $4.21(\mathrm{~d}, J=12.1 \mathrm{~Hz}, 1 \mathrm{H}), 3.40-3.30(\mathrm{~m}, 1 \mathrm{H}), 2.62(\mathrm{~d}, J=12.7 \mathrm{~Hz}, 1 \mathrm{H}), 2.13(\mathrm{~d}, J=9.5 \mathrm{~Hz}, 1 \mathrm{H})$, $1.59(\mathrm{t}, J=9.4 \mathrm{~Hz}, 3 \mathrm{H}), 0.74-0.67(\mathrm{~m}, 3 \mathrm{H}) . ;{ }^{13} \mathrm{C} \mathrm{NMR}\left(151 \mathrm{MHz}, \mathrm{CDCl}_{3}\right) \delta 159.2,156.8,154.9$, $138.0,134.3,134.0,133.5,132.0,131.9,131.0,130.7,129.7,129.6,129.4,129.0,128.7,128.5$, 
$128.2,128.0,127.9,126.9,126.4,77.5,74.7,68.9,32.4,31.6,25.7,23.3,15.3 ;{ }^{19} \mathrm{~F}$ NMR $(565$

$\left.\mathrm{MHz}, \mathrm{CDCl}_{3}\right) \delta-153.42,-153.37$; FTIR: 2940, 1618, 1561, 1102, 975, $702 \mathrm{~cm}^{-1}$; HRMS (ESI $\left.{ }^{+}\right)$

$\left[\mathrm{M}-\mathrm{BF}_{4}\right]^{+}$calculated for $\mathrm{C}_{36} \mathrm{H}_{34} \mathrm{NO}: 496.2635$, found 496.2640 .

\section{Mechanistic Experiments}

\section{Radical Trap Experiment}
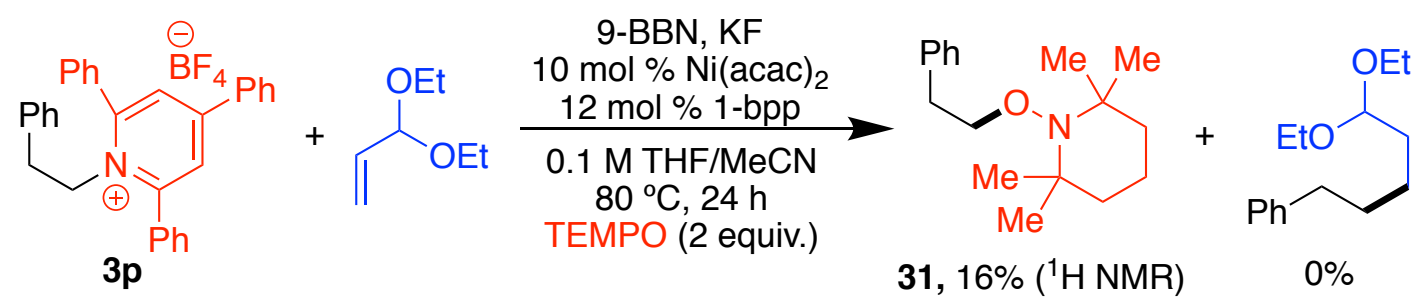

In a $\mathrm{N}_{2}$-atmosphere glovebox, 9-BBN ( $0.5 \mathrm{M}$ in THF, $0.5 \mathrm{~mL}, 0.25 \mathrm{mmol}, 2.5$ equiv), $\mathrm{KF}$ ( $16 \mathrm{mg}$, $0.275 \mathrm{mmol}, 2.75$ equiv), and alkene ( $38 \mu \mathrm{L}, 0.25 \mathrm{mmol}, 2.5$ equiv) were stirred in an oven-dried 1-dram vial fitted with a stir bar at $80^{\circ} \mathrm{C}$ in an aluminum heating block for 30 minutes. Meanwhile, $\mathrm{Ni}$ (acac) 2 (2.6 mg, $0.010 \mathrm{mmol}, 10 \mathrm{~mol} \%)$ and 1-bpp (2.5 mg, 0.012, $12 \mathrm{~mol} \%)$ were stirred in $\mathrm{MeCN}(0.5 \mathrm{~mL})$ at room temperature for 15 minutes. Then pyridinium salt $3 \mathbf{p}(50 \mathrm{mg}, 0.10 \mathrm{mmol}$, 1.0 equiv) and TEMPO (31 mg, $0.20 \mathrm{mmol}, 2.0$ equiv) were added to the vial containing 9-BBN, alkene, and KF. The nickel/ligand solution was added, the vial was capped with a Teflon-lined cap and removed from the glovebox. The mixture was stirred at $80{ }^{\circ} \mathrm{C}$ in an aluminum heating block for $24 \mathrm{~h}$. The mixture was then diluted with $\mathrm{Et}_{2} \mathrm{O}(3 \mathrm{~mL})$ and filtered through a plug of silica gel, which was rinsed with $\mathrm{Et}_{2} \mathrm{O}(10 \mathrm{~mL})$. The filtrate was concentrated. 1,3,5-Trimethoxybenzene (internal standard) was added. The yield of the known TEMPO adduct product $\mathbf{3 1}^{11}$ was determined to be $16 \%$ by ${ }^{1} \mathrm{H}$ NMR analysis. No cross-coupled product was observed. 


\section{Radical Clock Experiment}

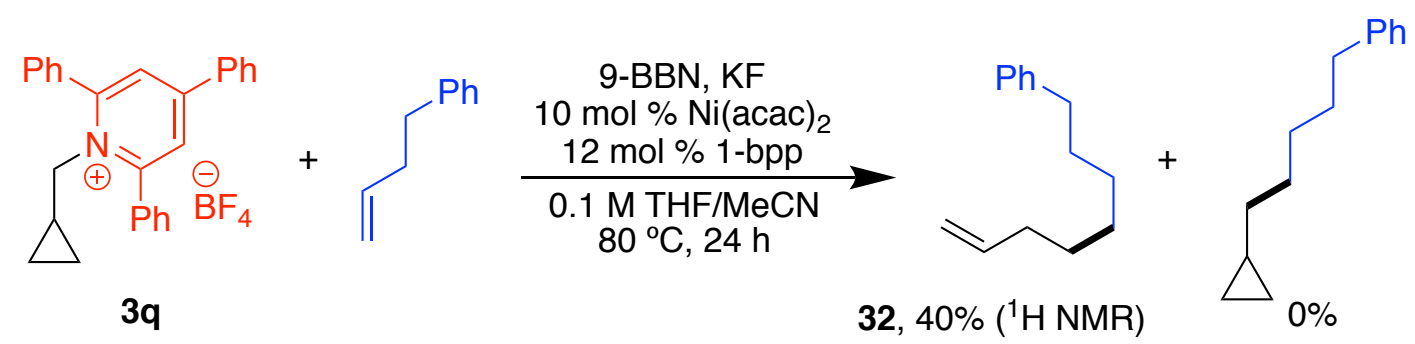

In a $\mathrm{N}_{2}$-atmosphere glovebox, 9-BBN (0.5 M in THF, $0.5 \mathrm{~mL}, 0.25 \mathrm{mmol}, 2.5$ equiv), KF (16 mg, $0.275 \mathrm{mmol}, 2.75$ equiv), and alkene ( $38 \mu \mathrm{L}, 0.25 \mathrm{mmol}, 2.5$ equiv) were stired in an oven-dried 1 -dram vial fitted with a stir bar at $80^{\circ} \mathrm{C}$ in an aluminum heating block for 30 minutes. Meanwhile, $\mathrm{Ni}(\mathrm{acac})_{2}(2.6 \mathrm{mg}, 0.010 \mathrm{mmol}, 10 \mathrm{~mol} \%)$ and 1-bpp (2.5 mg, 0.012, $\left.12 \mathrm{~mol} \%\right)$ were stirred in $\mathrm{MeCN}(0.5 \mathrm{~mL})$ at room temperature for 15 minutes. Then pyridinium salt $\mathbf{3 q}(45 \mathrm{mg}, 0.10 \mathrm{mmol}$, 1.0 equiv) was added to the vial containing 9-BBN, alkene, and KF. The nickel/ligand solution was added, the vial was capped with a Teflon-lined cap and removed from the glovebox. The mixture was stirred at $80{ }^{\circ} \mathrm{C}$ in an aluminum heating block for $24 \mathrm{~h}$. The mixture was then diluted with $\mathrm{Et}_{2} \mathrm{O}(3 \mathrm{~mL})$ and filtered through a plug of silica gel, which was rinsed with $\mathrm{Et}_{2} \mathrm{O}(10 \mathrm{~mL})$. The filtrate was concentrated. 1,3,5-Trimethoxybenzene (internal standard) was added. The yield of the known ring-opened product $32^{12}$ was determined to be $40 \%$ by ${ }^{1} \mathrm{H}$ NMR analysis. No cyclopropyl product was observed.

\section{References}

1. Pangborn, A. B.; Giardello, M. A.; Grubbs, R. H.; Rosen, R. K.; Timmers, F. J., Safe and convenient procedure for solvent purification. Organometallics 1996, 15 (5), 1518-1520.

2. Brown, H. C.; Chen, J., Hydroboration. 57. Hydroboration with 9-borabicyclo[3.3.1]nonane of alkenes containing representative functional groups. The Journal of Organic Chemistry 1981, 46 (20), 3978-3988.

3. Chen, H.; Sun, S.; Liao, X., Nickel-Catalyzed Decarboxylative Alkenylation of Anhydrides with Vinyl Triflates or Halides. Organic Letters 2019, 21 (10), 3625-3630.

4. Zhou, Q.-Q.; Düsel, S. J. S.; Lu, L.-Q.; König, B.; Xiao, W.-J., Alkenylation of unactivated alkyl bromides through visible light photocatalysis. Chemical Communications 2019, 55 (1), 107-110.

5. Silvia Roscales, I. G. S., Aurelio G. Csaky, Regioselective 1,6-Conjudate Addition of Boronic Acids and Grignard Reagents to Dienylpyridines. Synlett 2011, 15, 2234-2236.

6. Plunkett, S.; Basch, C. H.; Santana, S. O.; Watson, M. P., Harnessing Alkyl Pyridinium Salts as Electrophiles in De-aminative Alkyl-Alkyl Cross-Couplings. J. Am. Chem. Soc. 2019, 141 (6), $2257-2262$.

7. Liao, J.; Basch, C. H.; Hoerrner, M. E.; Talley, M. R.; Boscoe, B. P.; Tucker, J. W.; Garnsey, M. R.; Watson, M. P., Deaminative Reductive Cross-Electrophile Couplings of Alkylpyridinium Salts and Aryl Bromides. Org. Lett. 2019, 21 (8), 2941-2946.

8. Liao, J.; Guan, W.; Boscoe, B. P.; Tucker, J. W.; Tomlin, J. W.; Garnsey, M. R.; Watson, M. P., Transforming Benzylic Amines into Diarylmethanes: Cross-Couplings of Benzylic Pyridinium Salts via C-N Bond Activation. Org. Lett. 2018, 20 (10), 3030-3033. 
9. Basch, C. H.; Liao, J.; Xu, J.; Piane, J. J.; Watson, M. P., Harnessing Alkyl Amines as Electrophiles for Nickel-Catalyzed Cross Couplings via C-N Bond Activation. J. Am. Chem. Soc. 2017, 139 (15), 5313-5316.

10. Wu, J.; He, L.; Noble, A.; Aggarwal, V. K., Photoinduced Deaminative Borylation of Alkylamines. J. Am. Chem. Soc. 2018, 140 (34), 10700-10704.

11. Patel, V. F.; Pattenden, G., Free radical reactions in synthesis. Homolysis of alkylcobalt complexes in the presence of radical-trapping agents. Journal of the Chemical Society, Perkin Transactions 1 1990, (10), 2703-2708.

12. Uemura, M.; Yorimitsu, H.; Oshima, K., Synthesis of $\mathrm{Cp}^{*} \mathrm{CH} 2 \mathrm{PPh} 2$ and its use as a ligand for the nickelcatalysed cross-coupling reaction of alkyl halides with aryl Grignard reagents. Chemical Communications 2006, (45), 4726-4728. 


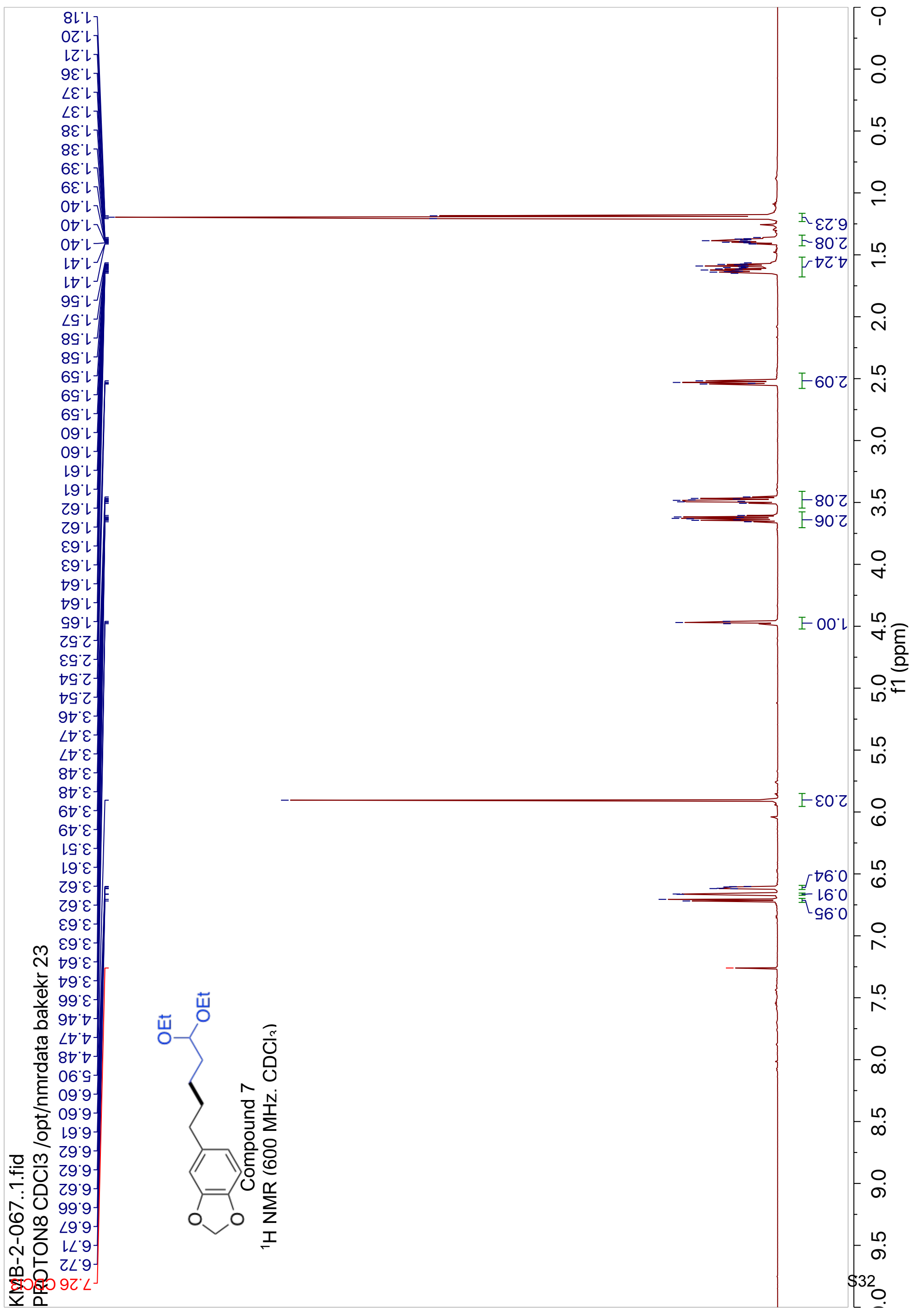




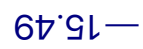

カヤナてー

$9 \angle ' L$

$\angle G \cdot \varepsilon \varepsilon-$

乙L $\mathcal{G}^{-}$

ยリ๐つ $\nabla 8^{\circ} 9<$

हlอवว $9 l^{\circ} \angle L=$

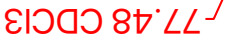

$28.00 \mathrm{~L}-$

$\angle 6$ ZOL

$\angle \mathrm{L} 80 \mathrm{~L}$

$86.801=$

LlレZLー

09.9عL-

ด

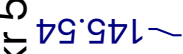

d $\angle 9^{\circ} \angle \nabla l-$

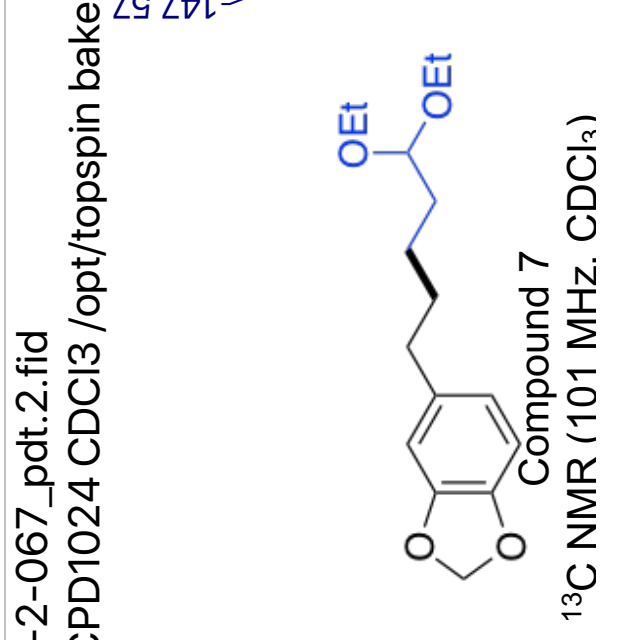

으ำ

粞

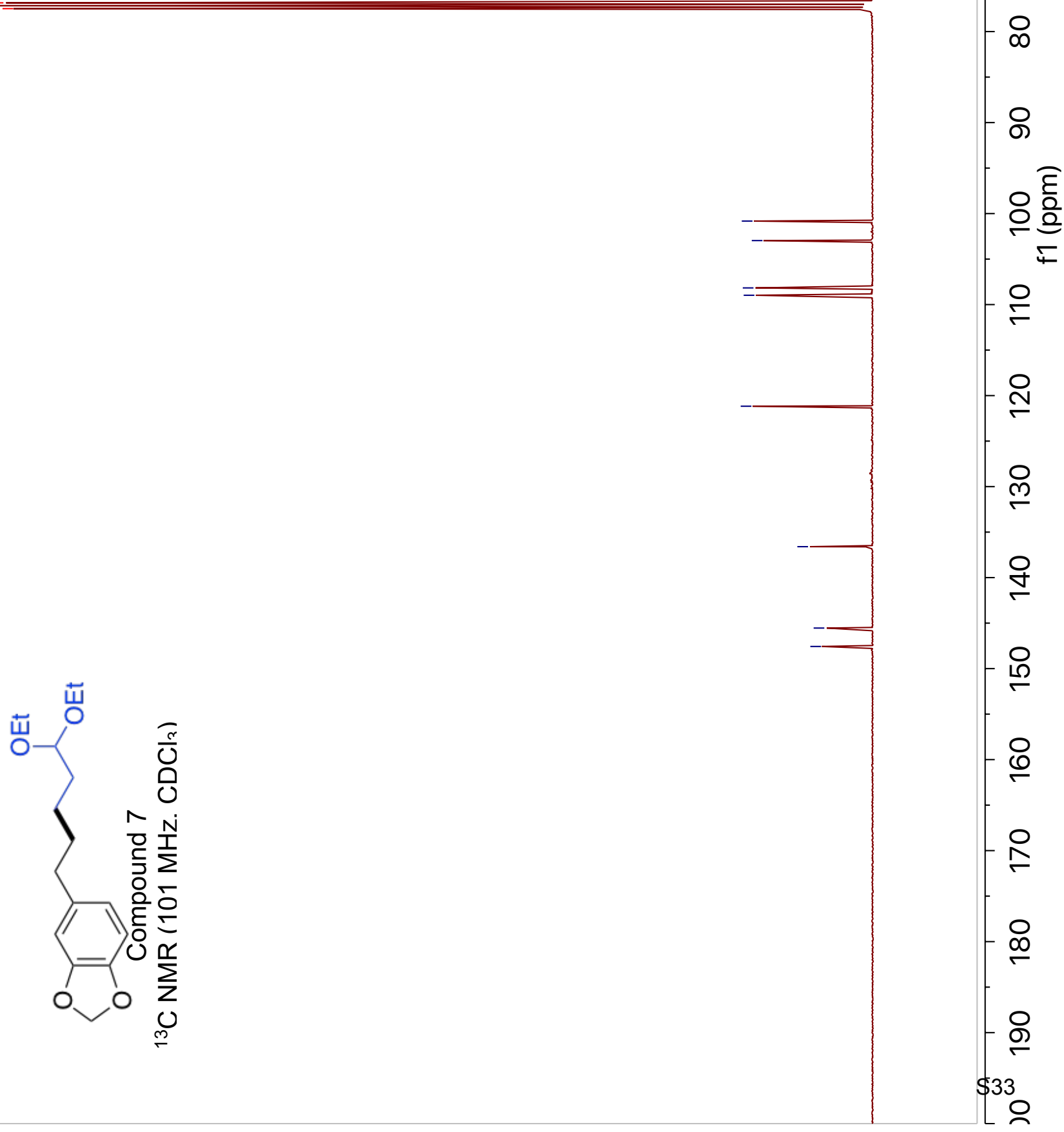




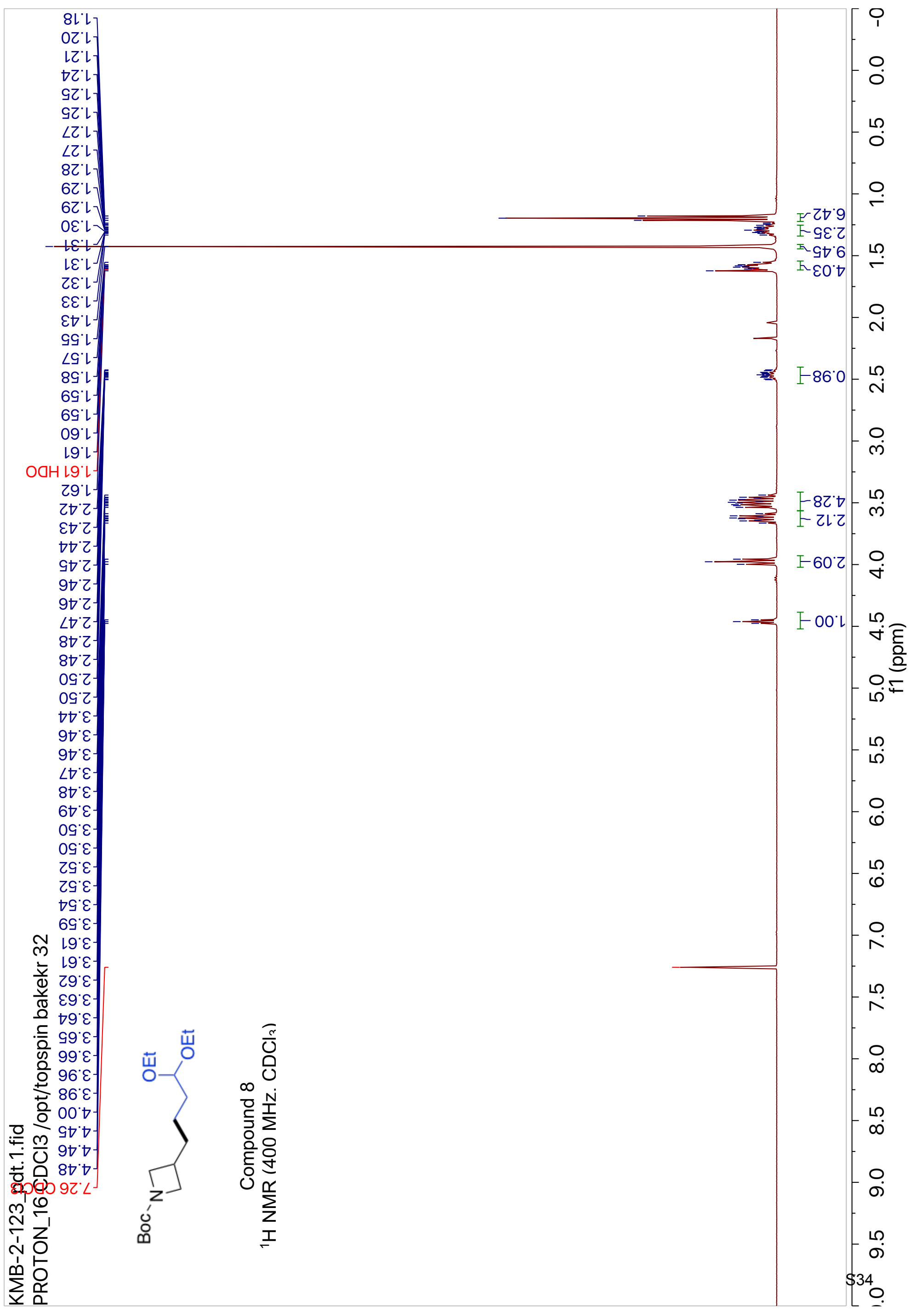


679ட-

๕๕てて99: 82

96.82 -

ZL'LE-

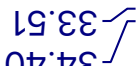

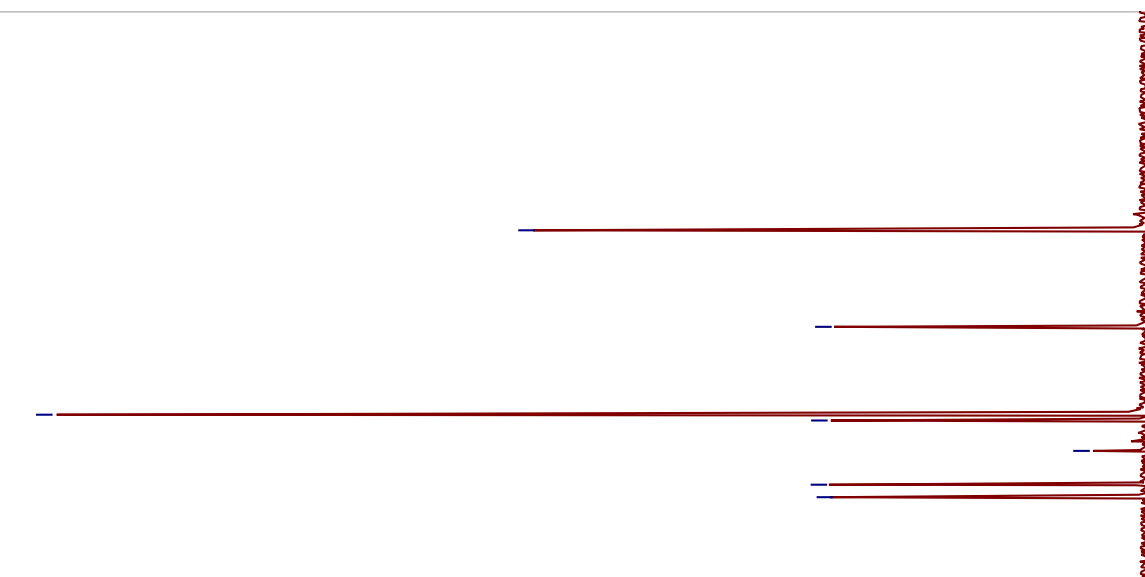

ㄴ․ㅇ-

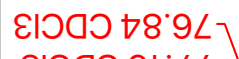

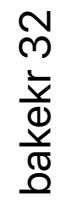
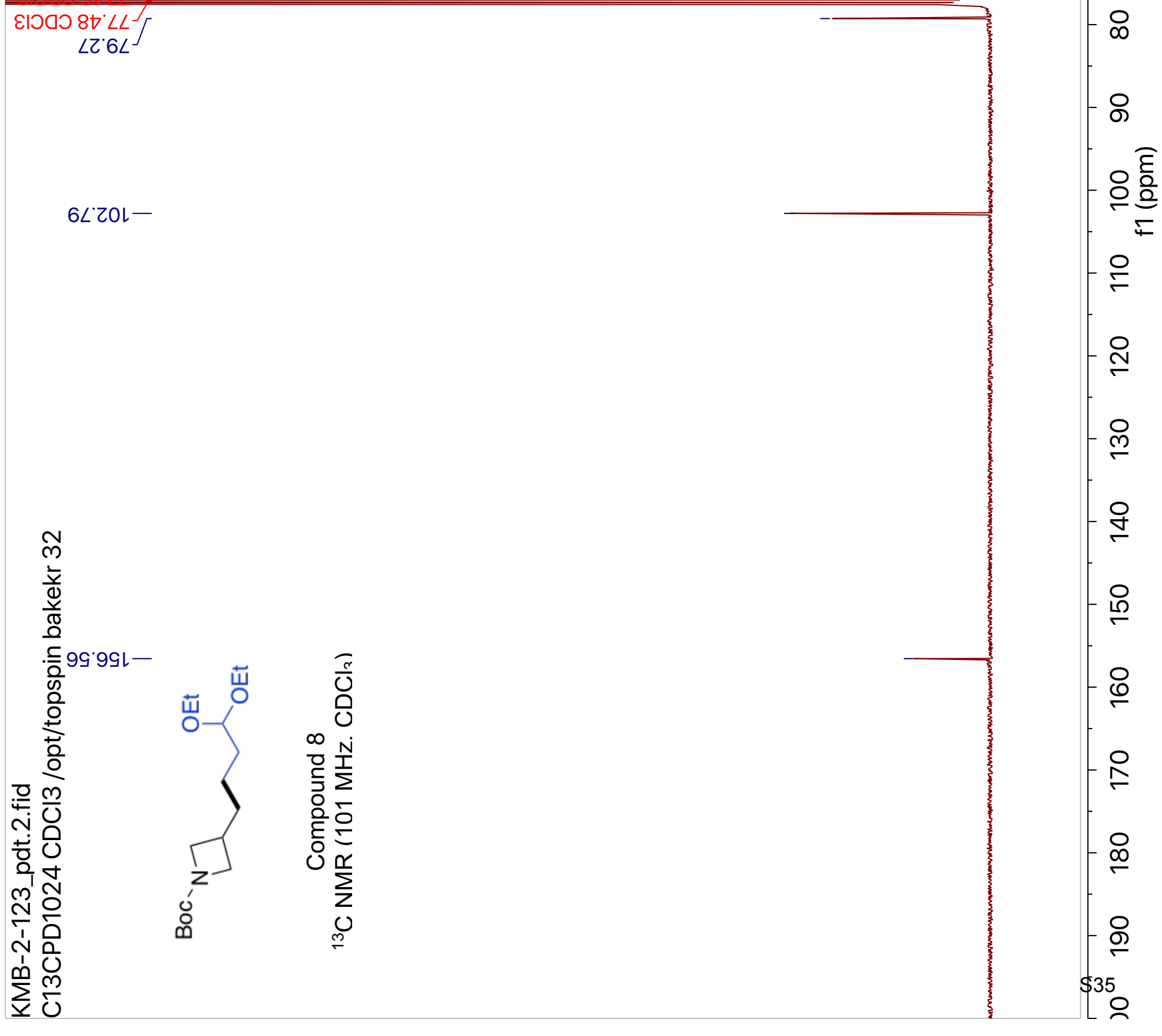


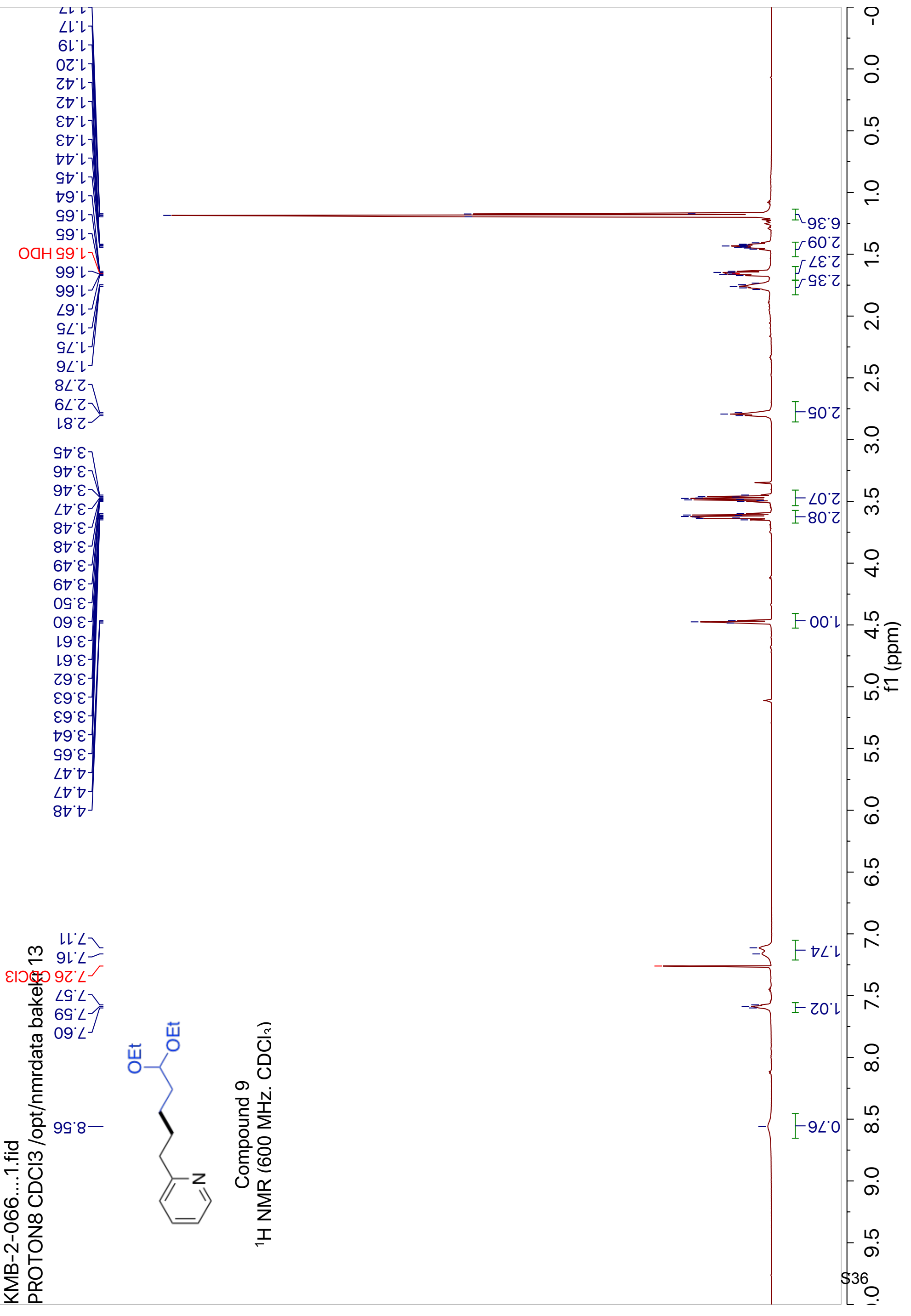


8tஒงL-

99.七て

98.6乙-

$89^{\circ} \varepsilon \varepsilon-$

$6 \varepsilon^{\circ} 8 \varepsilon \backslash$

$70.19-$

$-8$

ยเวดว $\nabla 8^{\circ} 9 \angle$

(1)

ElO0J 8t $\angle L$

E6てOLー

UレレてL

ย6ててレー

6t'9عL-

$\stackrel{\bigcirc}{\checkmark}$

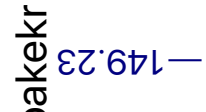

응

믄

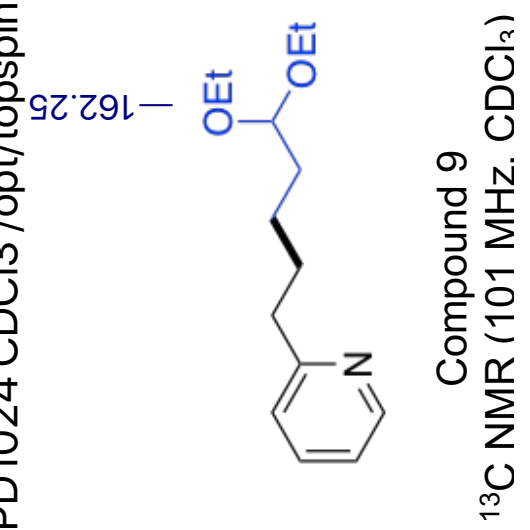

늠

$\sum_{\substack{0 \\ 0}}^{m}$

$\stackrel{0}{1}$

잉

우

응 응

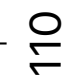

- ำ

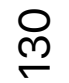

$\stackrel{0}{ \pm}$

$\stackrel{ }{ }$

$\stackrel{0}{6}$

읃

$\stackrel{\infty}{\infty}$

음 

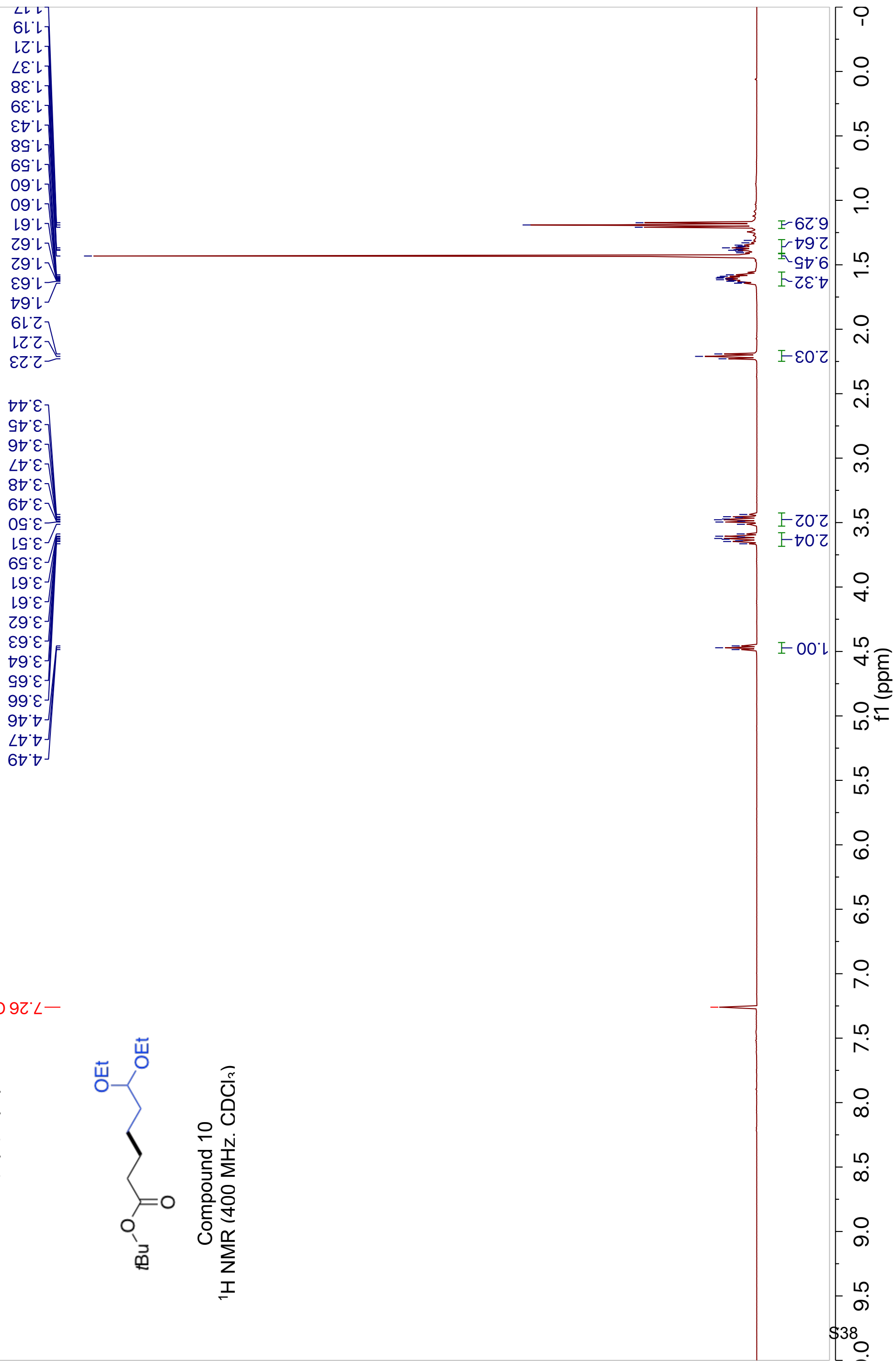
679ட-

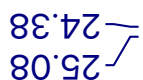
$\nabla Z^{\circ} 8 \tau^{\top}$

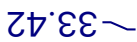

$\neg 9^{\circ} 9 \varepsilon-$

†0.19-

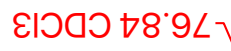

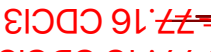

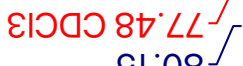

Gl.08

\&8'ZOL-

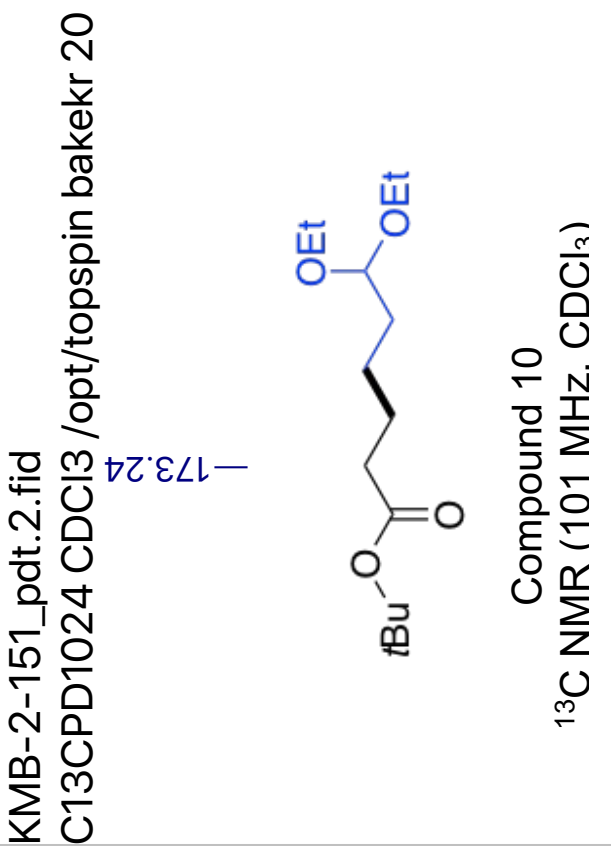

I

윰

-

$\stackrel{\circ}{2}$

-

- 음 


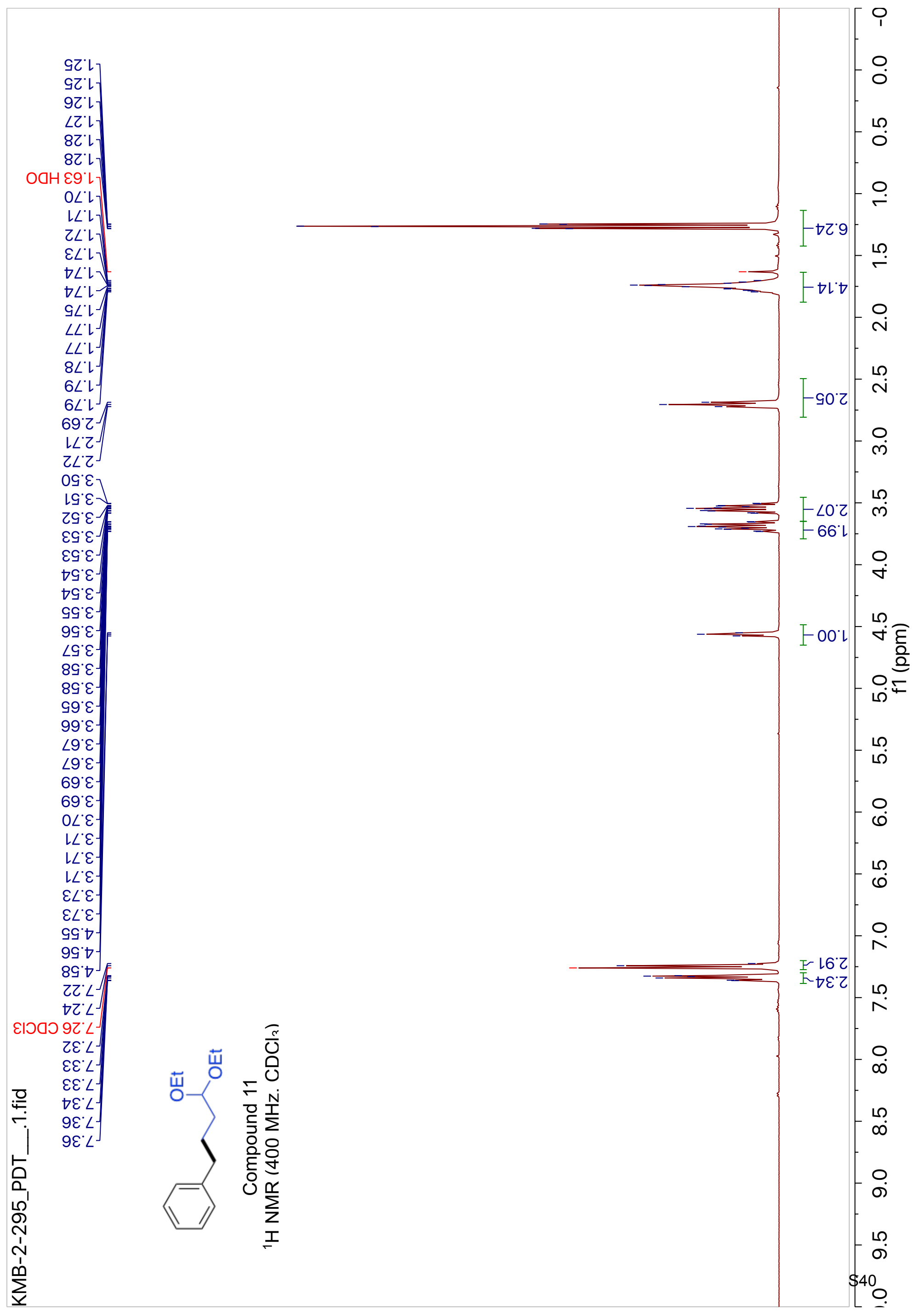


OG'GL-

$9 L 92-$

$8 \varepsilon^{\circ} \varepsilon \varepsilon-$

98. $9 \varepsilon-$

$60.19-$

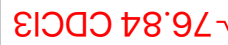

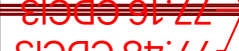

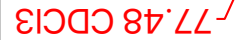

96 ZOL-

989Zレ

てヤ・8ZL

99.8ZL

くヤでレー

ํㅜㄴ

พุ.

ஸ่
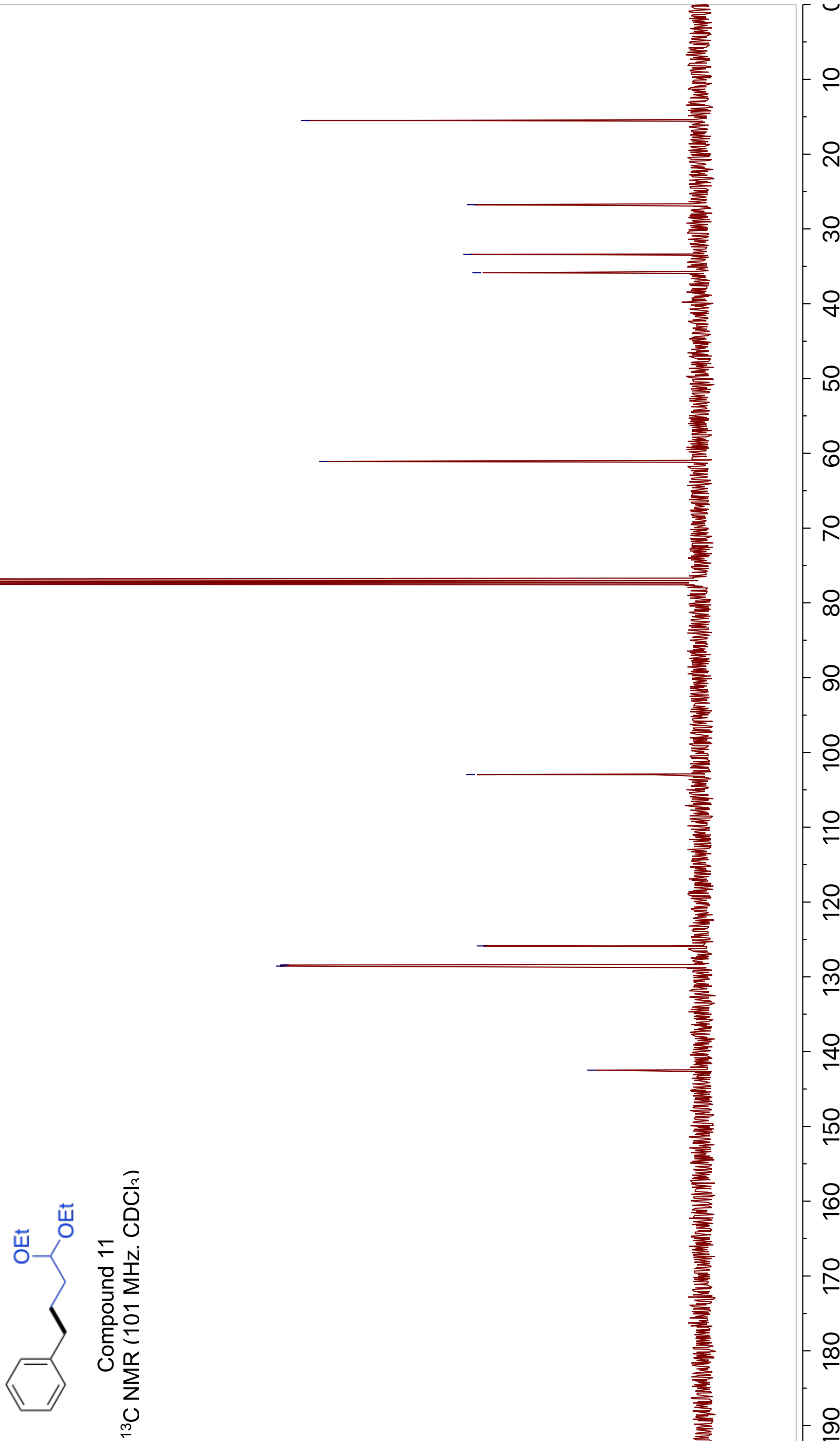

웅

8

ㅇ

$\infty$

ㅇ

으르을

을

$\stackrel{ }{\sim}$

음

$\stackrel{ }{Ð}$

음

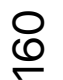

$\sum_{\Sigma}^{\infty} \sum^{\infty}$ 


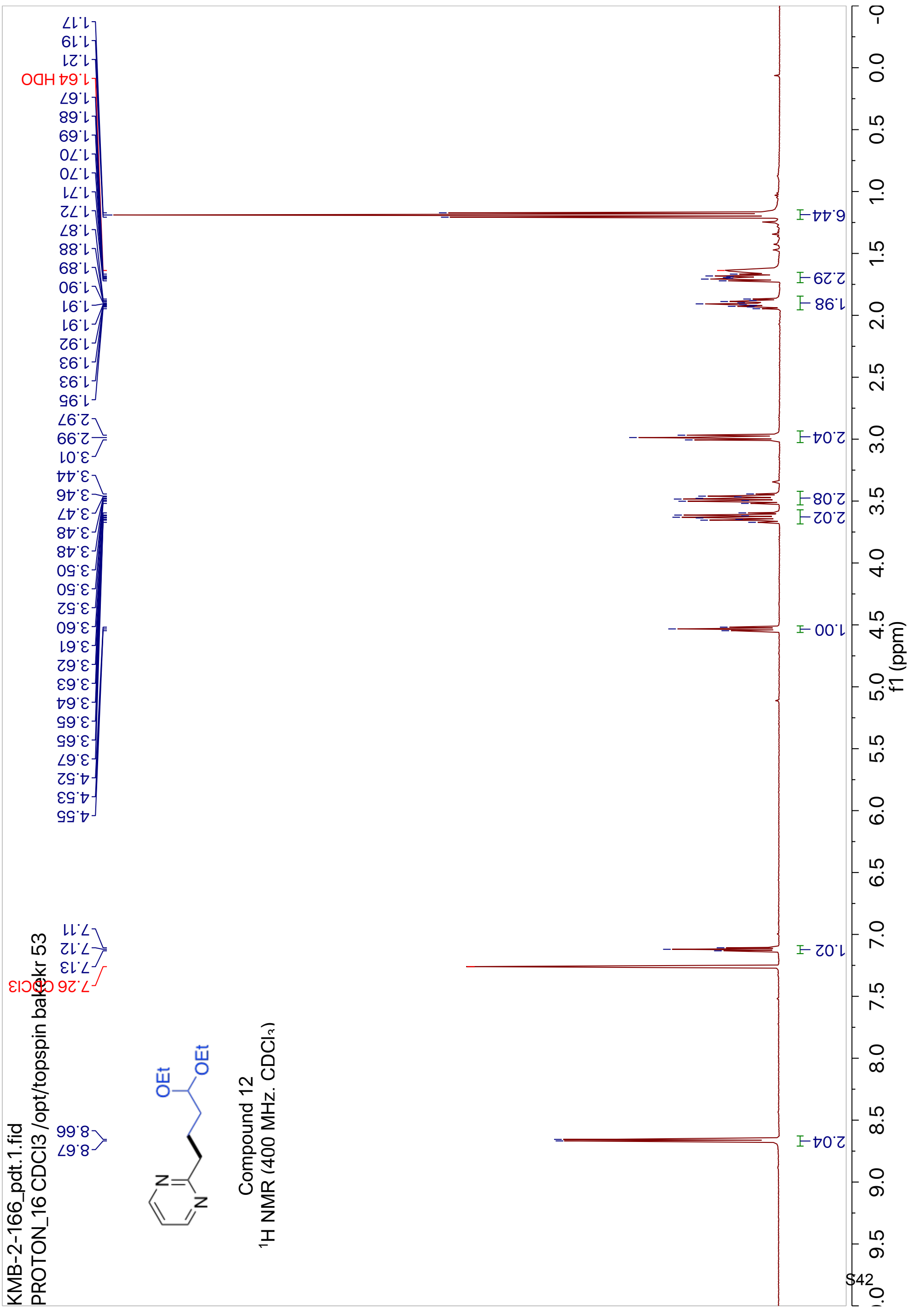


679 9 -

ง6'ย乙-

乙ย'ยะ-

乙ย'6ع-

80.19-

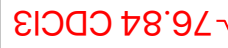

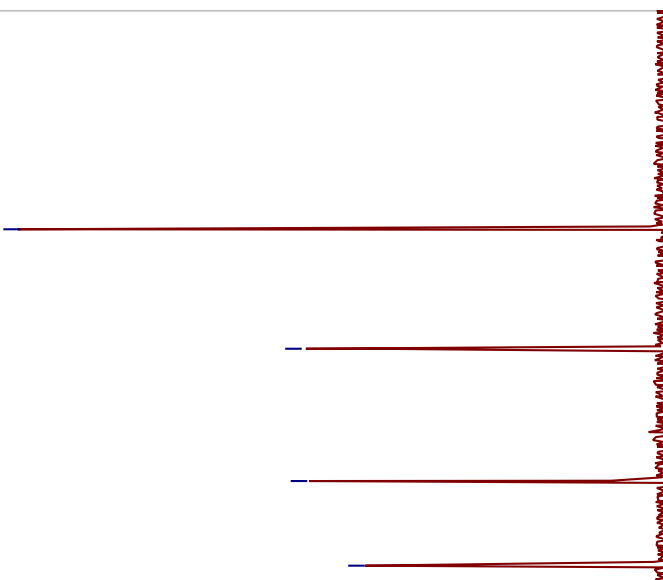

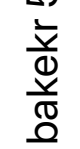

등

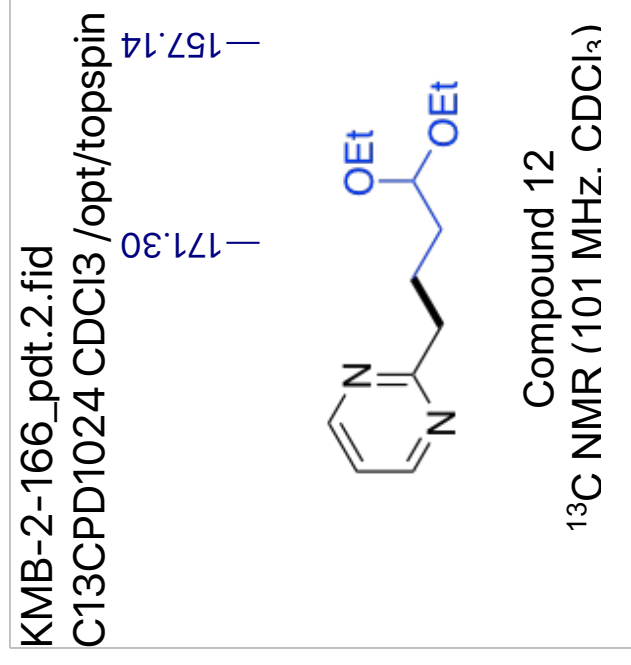




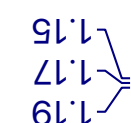

29.

E9.1-

$\nabla 9^{\circ} \mathrm{L}$

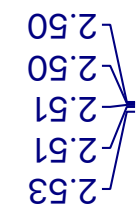

$6 \varepsilon^{\circ} \varepsilon$

O० $\varepsilon$

$\left\llcorner\nabla^{\circ} \varepsilon\right.$

乙ฤ $\varepsilon$

$\varepsilon \nabla^{\circ} \varepsilon$

$\nabla \nabla{ }^{\circ} \varepsilon$

$9 \nabla^{\circ} \varepsilon$

$9 \nabla^{\circ} \varepsilon$

$\nabla \mathcal{G}^{\circ} \varepsilon$

$99^{\circ} \varepsilon$

$99^{\circ} \varepsilon$

$\angle \mathcal{G}^{\circ} \mathcal{\varepsilon}$

$89^{\circ} \varepsilon$

$69^{\circ} \varepsilon$

$09^{\circ} \varepsilon$

$19^{\circ} \varepsilon$

$\angle \varepsilon^{\circ} \nabla$

$6 \varepsilon^{\circ} \nabla$

Ot

$\left.\begin{array}{l}62 \cdot 9 \\ 62 \cdot 9 \\ 0 \varepsilon \cdot 9\end{array}\right]$

9ட9

$9<\cdot 9>$

$\angle L \cdot 9$

으 $\varepsilon Z Z^{\circ}$

六 $\nabla Z^{\prime} L>$

흐 $9 L^{\circ} L$

عાग $92^{\circ} \angle$

흔 $L \varepsilon^{\circ} L$

응 $L \varepsilon^{\circ} L$

응 $\measuredangle \varepsilon ' L$

劳 $Z \varepsilon^{\circ} \angle$

으 음

은

ㅍํㅇ

ตै

ด

군

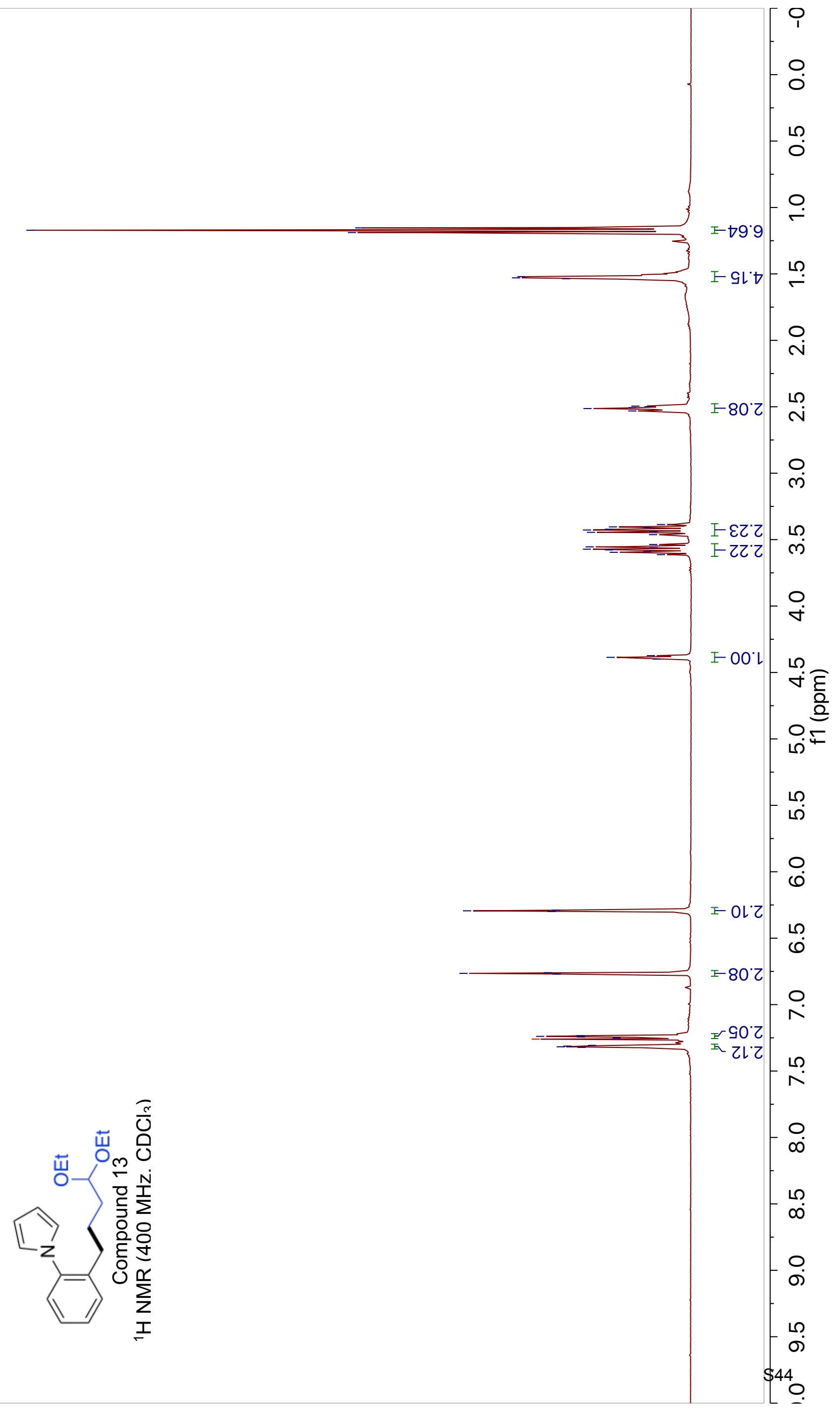


$\angle \nabla G L$

$10.92-$

68.0ह 久

$8 \varepsilon^{\circ} \varepsilon \varepsilon \sim$

10.19-

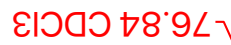

हा

$\angle 9^{\circ} \mathrm{ZOL}-$

28'80L-

97ててレ】

69.9ZL

$\varepsilon \varepsilon^{\circ} \angle Z L$

$\nabla 6{ }^{\circ} \angle Z L$

lloel

l9.8EL -

윤

高

음

등

음

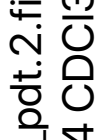

ำ

ㅇํㅇ

늠

$\sum^{\prime} \stackrel{m}{0}$

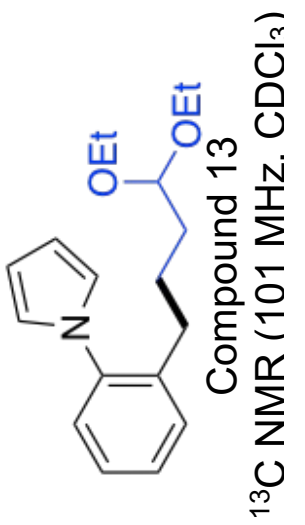

윰

8

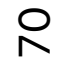

$\infty$

우

응 흥

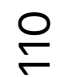

$\stackrel{ }{\sim}$

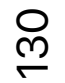

$\stackrel{ }{\Xi}$

$\stackrel{ }{ }$

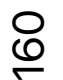

$\stackrel{ }{ }$

$\stackrel{\infty}{\infty}$

- 으 


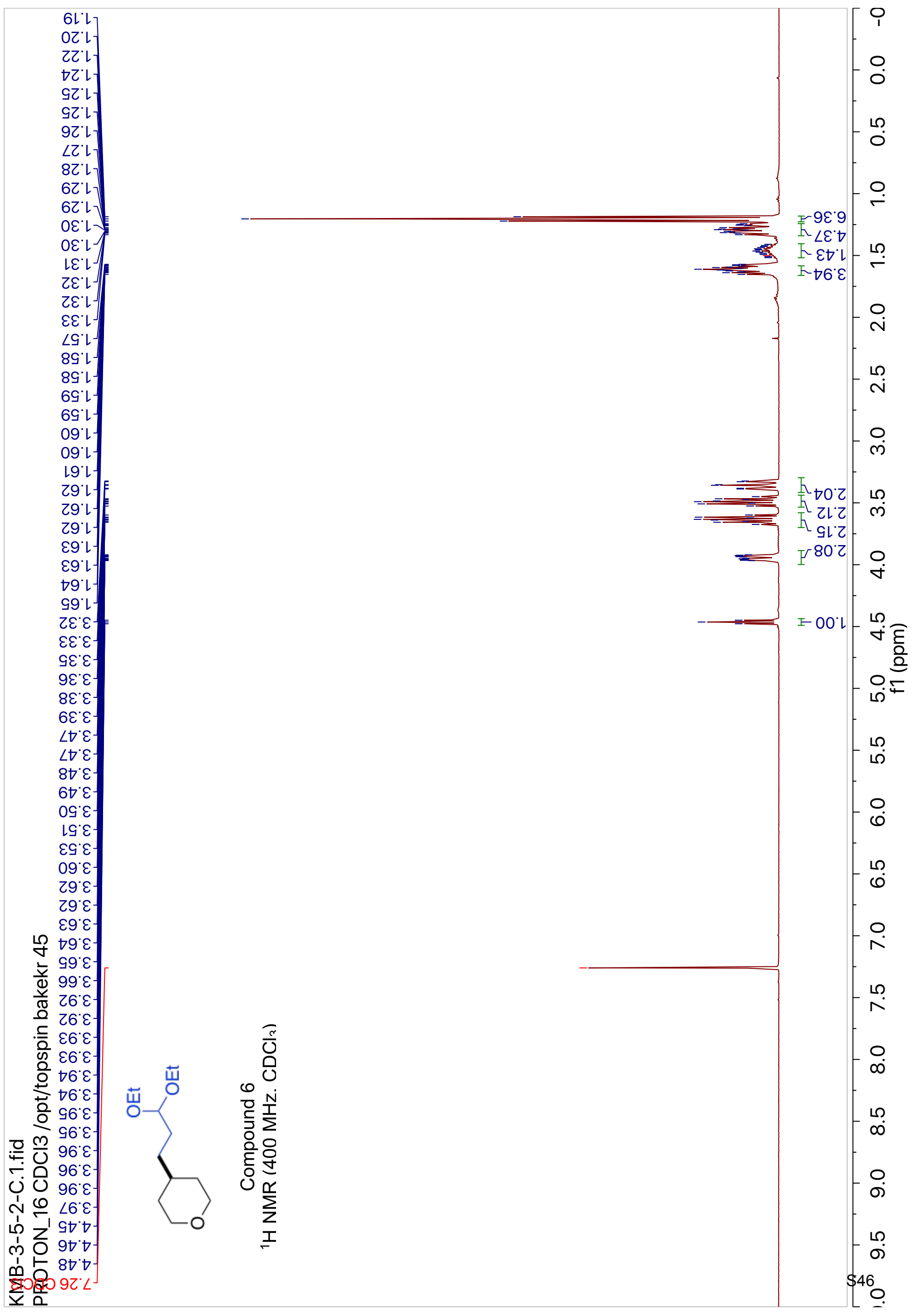


og'Gl-

$99^{\circ} 0 \varepsilon_{7}$

$\angle 6^{\circ} L E-$

$9 乙^{\circ} \varepsilon \varepsilon$

$\varepsilon 0^{\circ} \varsigma \varepsilon^{\top}$

$\angle 0^{\circ} 19-$

$92: 89-$

ยІวดว จ ${ }^{\circ} 9 L$

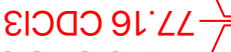

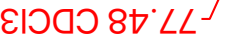

8เ'EOL-

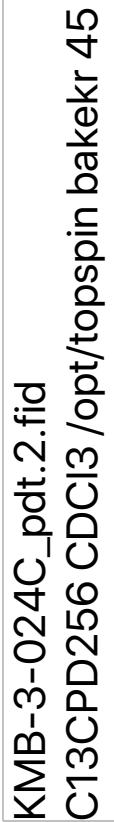
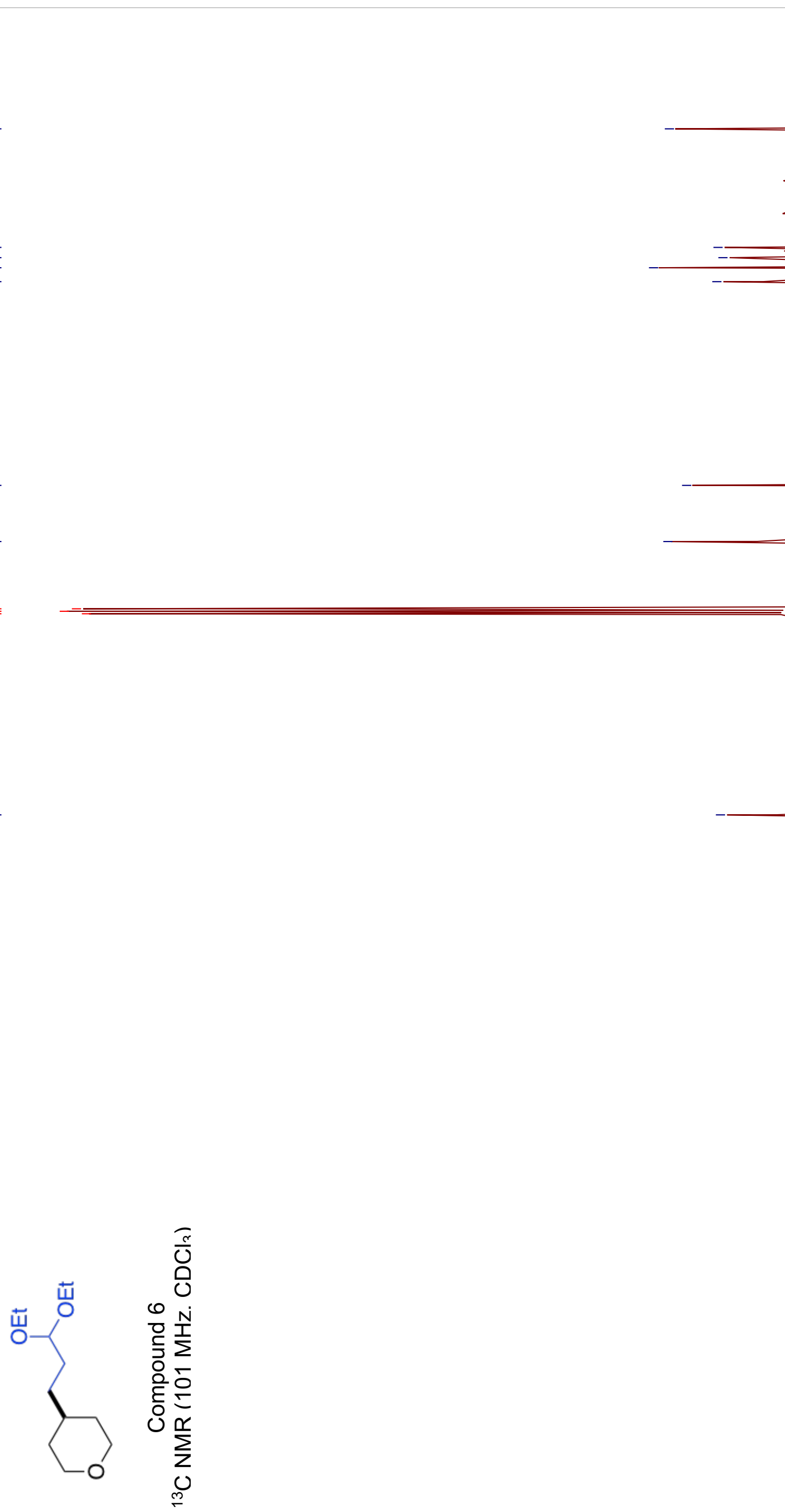

음

$\stackrel{\Upsilon}{\circ}$

ल

o

ำ

8

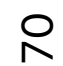

$\infty$

우

응 $\frac{\bar{\varepsilon}}{\mp}$

$-\frac{0}{2}$

ำ

$\stackrel{ }{\sim}$

$\stackrel{ }{\Xi}$

$\stackrel{ }{\circ}$

음

음

$\stackrel{\infty}{\infty}$

- 음 


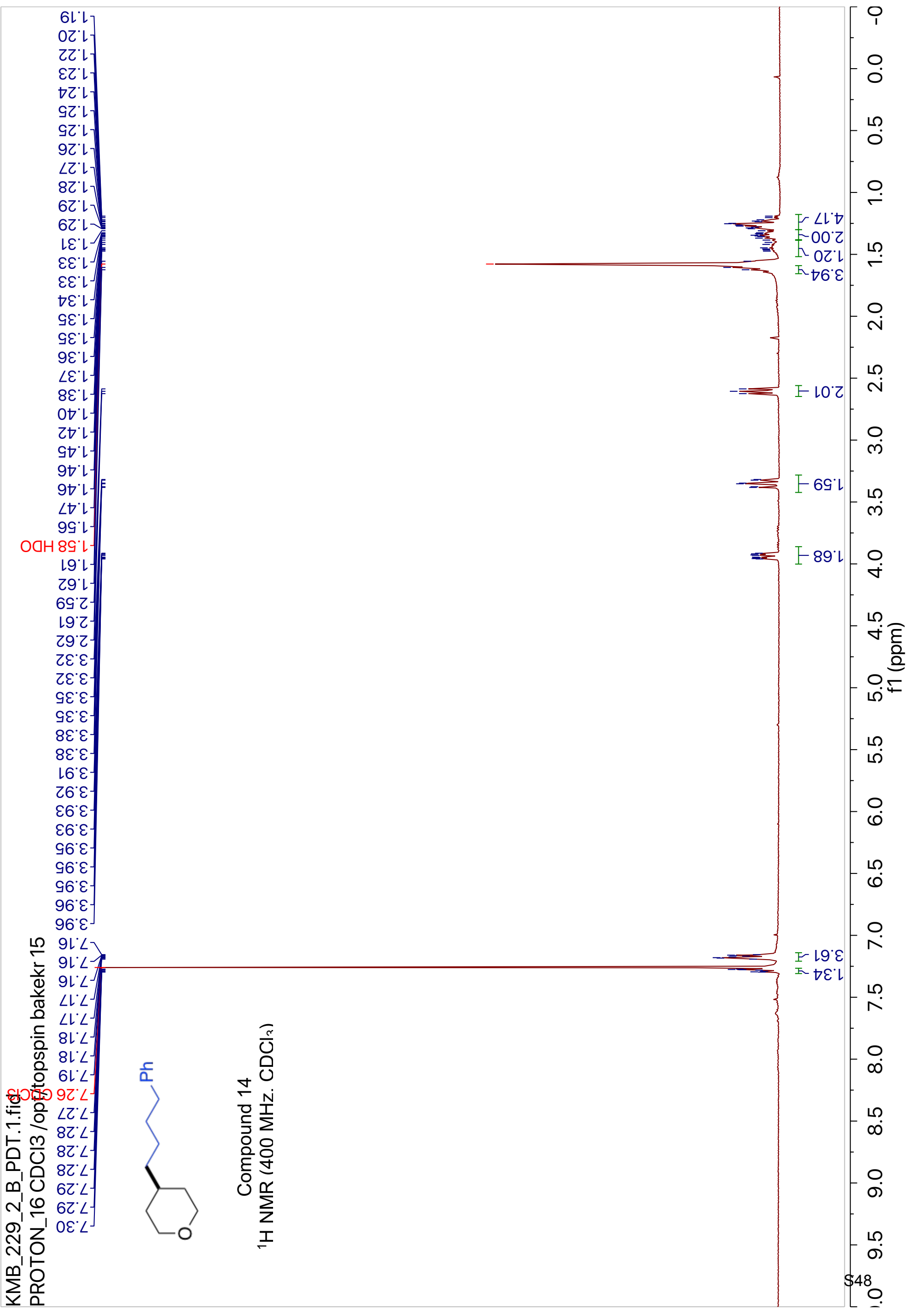


จ๑ \乙

$\angle \nabla^{\circ} L E$

$\varepsilon 0^{\circ} \varepsilon \varepsilon$

$\varepsilon \angle{ }^{\circ} \nabla \varepsilon-$

$9 L \cdot 9 \varepsilon-$

$29.9 \varepsilon^{\top}$

$10 \cdot 89-$

દાวดว દ9' $9 \angle$

हाวดว $9 l^{\circ} \angle L$

†๑ GZL

$\angle 0^{\circ} 8 Z \mathrm{Ll}$

0Z:8ZL
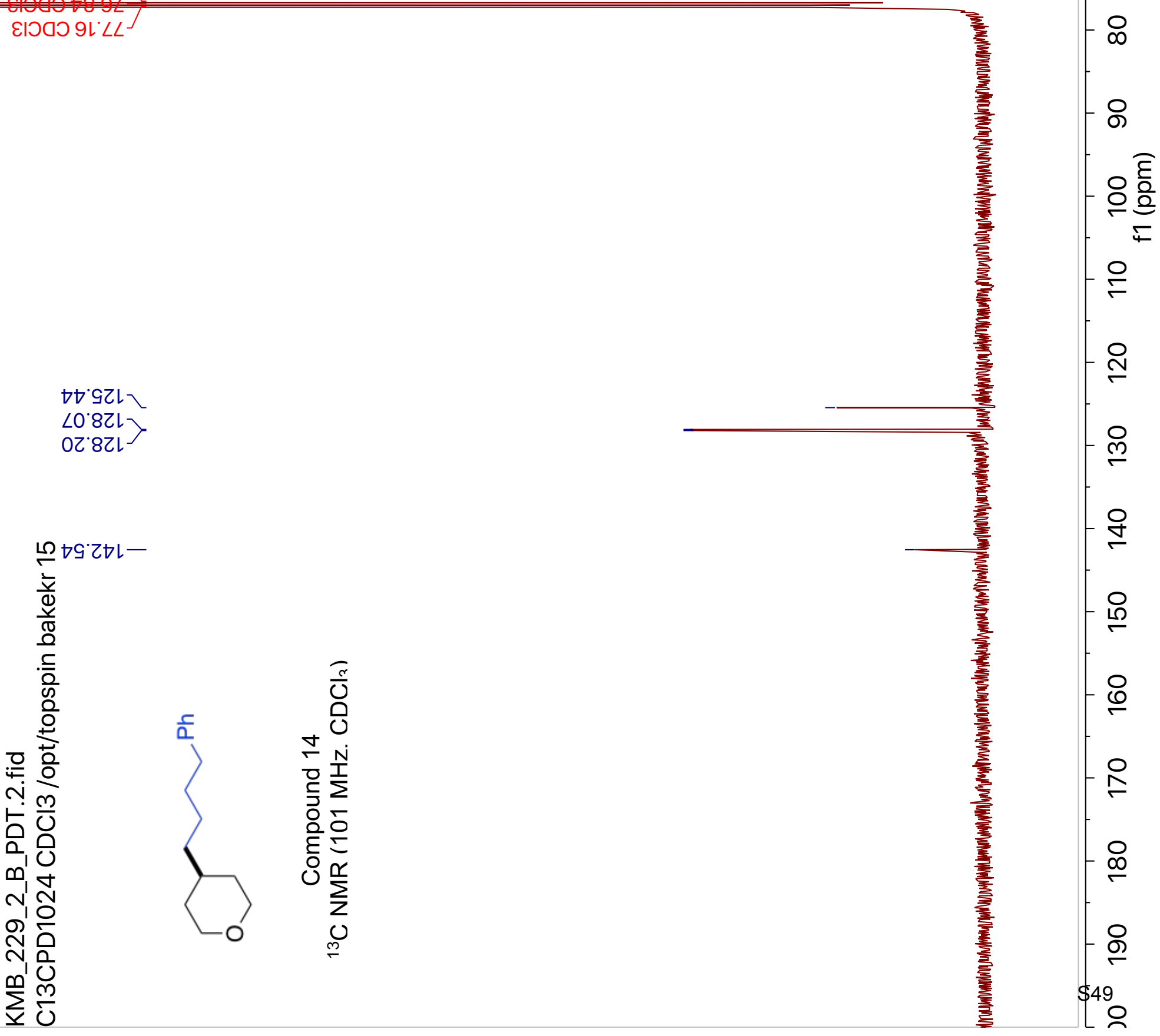


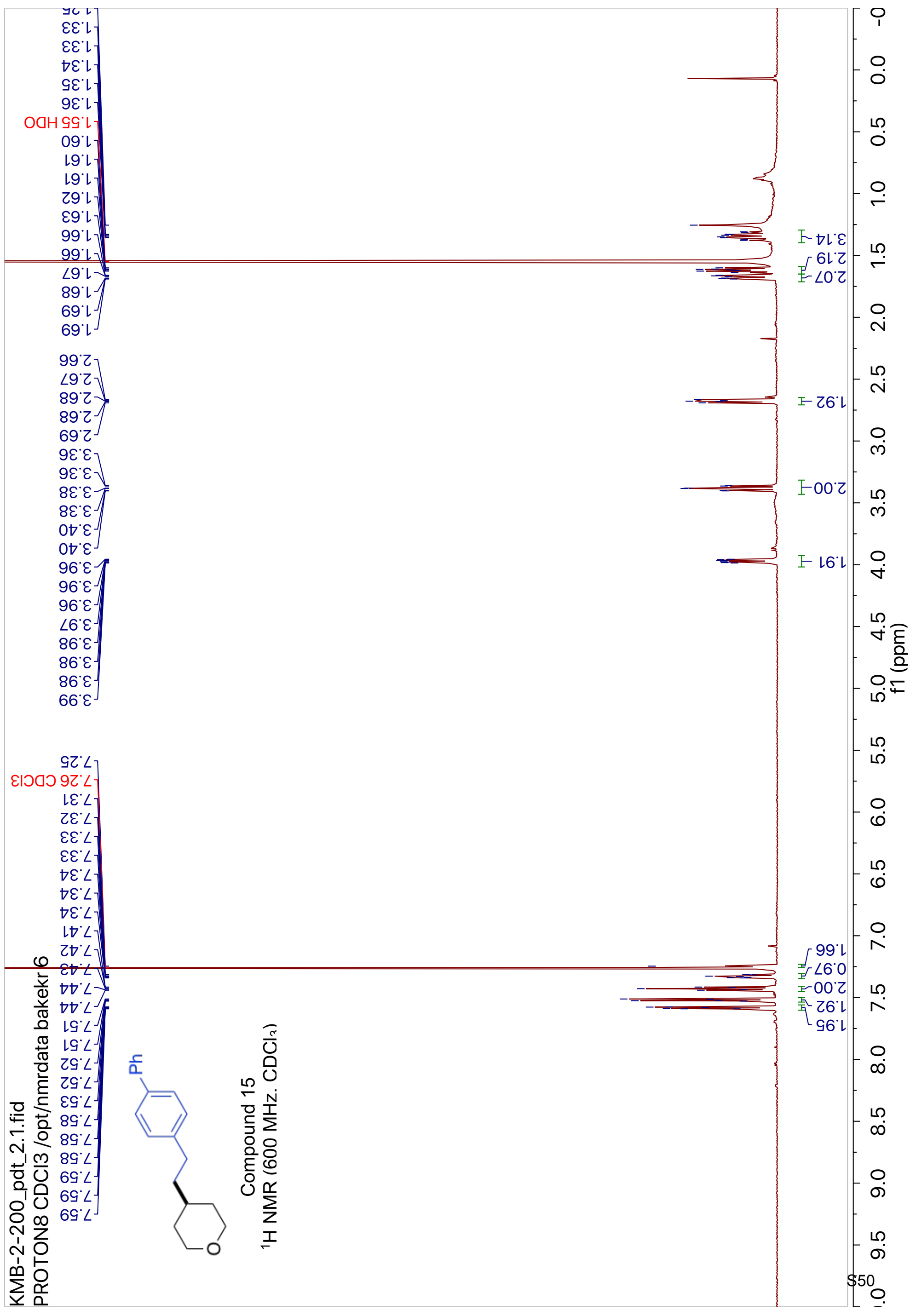


$00^{\circ} L \varepsilon_{-}$

เฉ'ฉE-

Ol' $\varepsilon \varepsilon$

$\nabla \mathcal{G}^{\circ} \nabla \varepsilon$

$\nabla \angle L^{\circ} 8 \varepsilon^{J}$

Ol'89-

ยเจว 乙L’ $9 \angle$

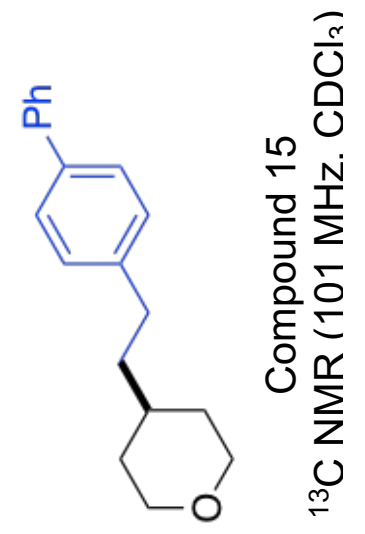




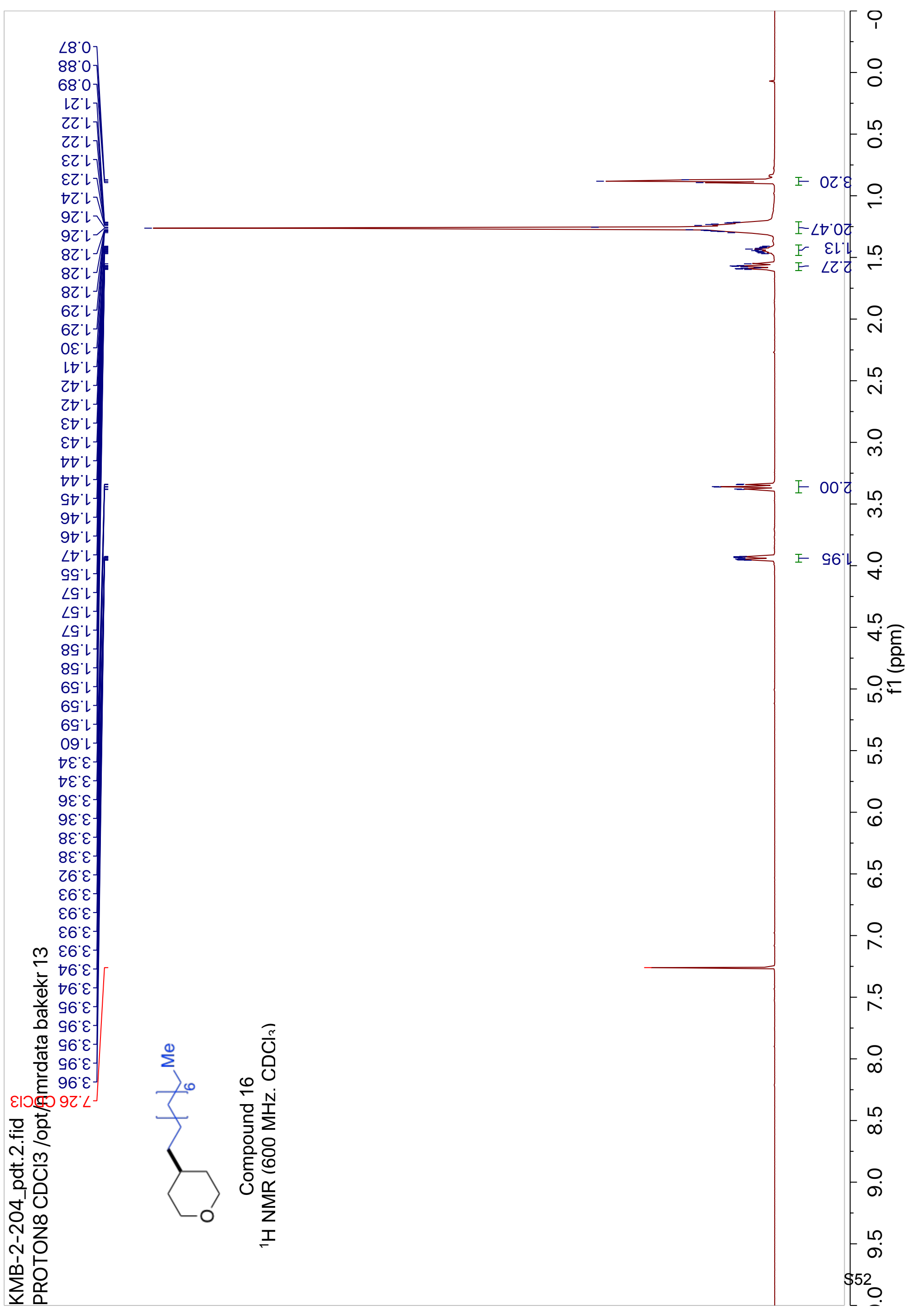


6でヤレー

$\triangleright 8^{\circ} \mathrm{Z}$

$09: 9 Z$

$09^{\circ} 6 z$

$6 \angle \cdot 6 z]$

28.62

$\angle 6.62$

ZL'LE

$90{ }^{\circ} Z \varepsilon$

$8 \varepsilon^{\prime} \varepsilon \varepsilon$

$60{ }^{\circ} 9 \varepsilon$

Zl:LE

$9 \varepsilon^{\circ} 89-$

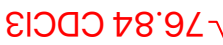

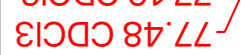

음
$\frac{1}{0}$
$\frac{1}{\pi}$
$\frac{0}{0}$
.$\frac{1}{0}$
$\frac{0}{0}$
$\frac{0}{0}$
$\frac{1}{1}$
0
0

믄 $\frac{m}{0}$

잉

워

ป ฐ

군

ㄴํㅁ

$\sum_{\Sigma}^{\infty} \frac{m}{u}$

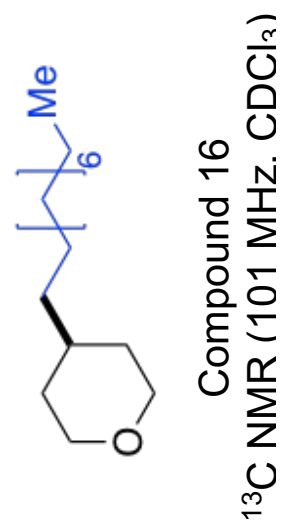




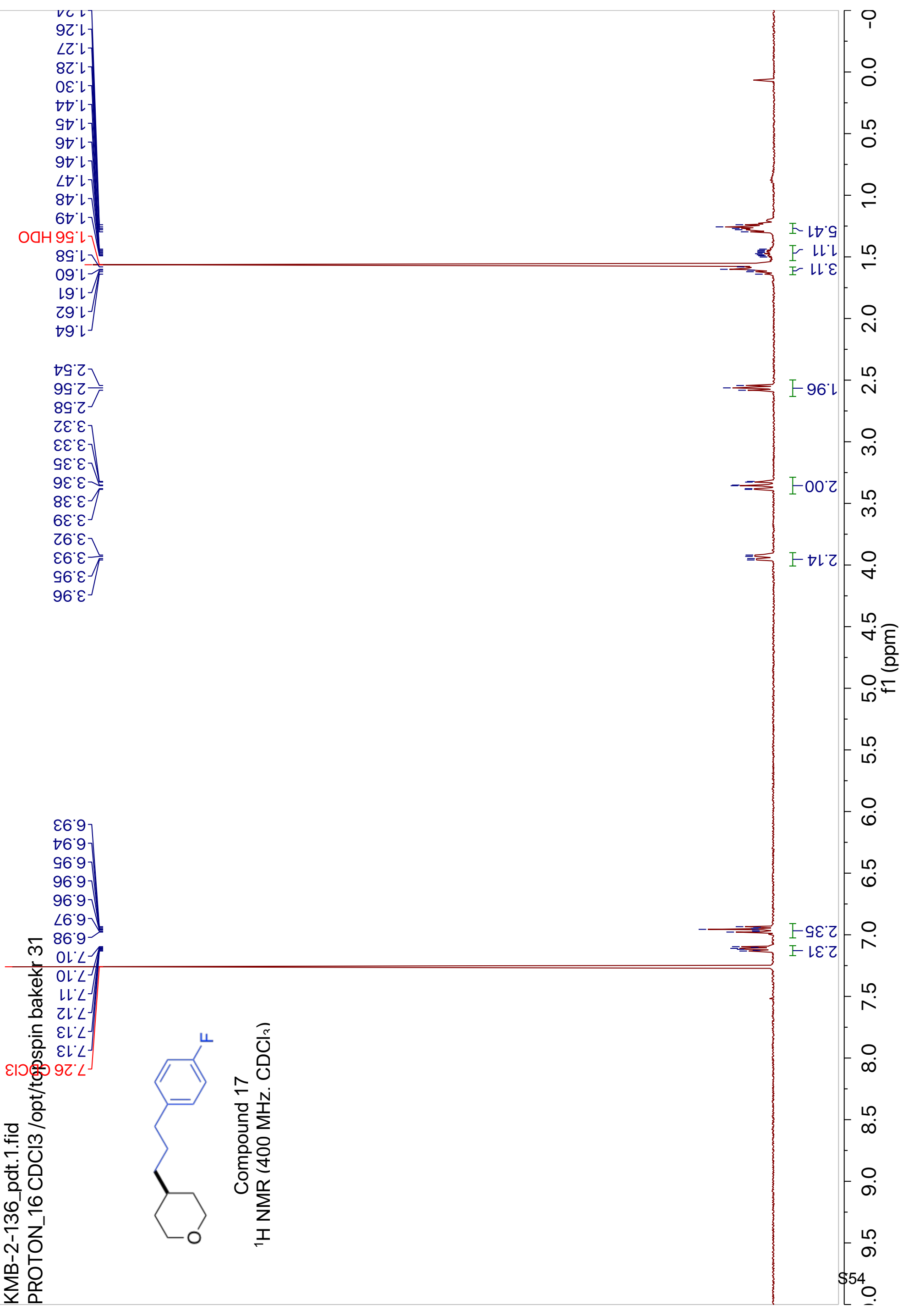


$89^{\circ} 82$

$8 乙^{\circ} \varepsilon \mathcal{E}$

$\varepsilon 0 \cdot 9 \varepsilon$ ᄂ

$6 \varepsilon^{\circ} 9 \varepsilon$

$69^{\circ} 9 \varepsilon^{\top}$

$82 \cdot 89-$

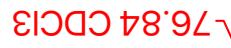

l0.9ll
Z2:Gll

ZL6ZL
$08 \cdot 6 Z L$

$\angle Z \circ 8 \varepsilon L$

$\bar{m}$

$0 \varepsilon^{\circ} 8 \varepsilon \downarrow-$

항

을

흥 $80.091-$

용ㅇ.091-

응

은 $\frac{m}{0}$

ำ

ㅍํㅇ

(1)

ํㅡㅇ

‘்

닌

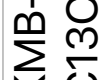
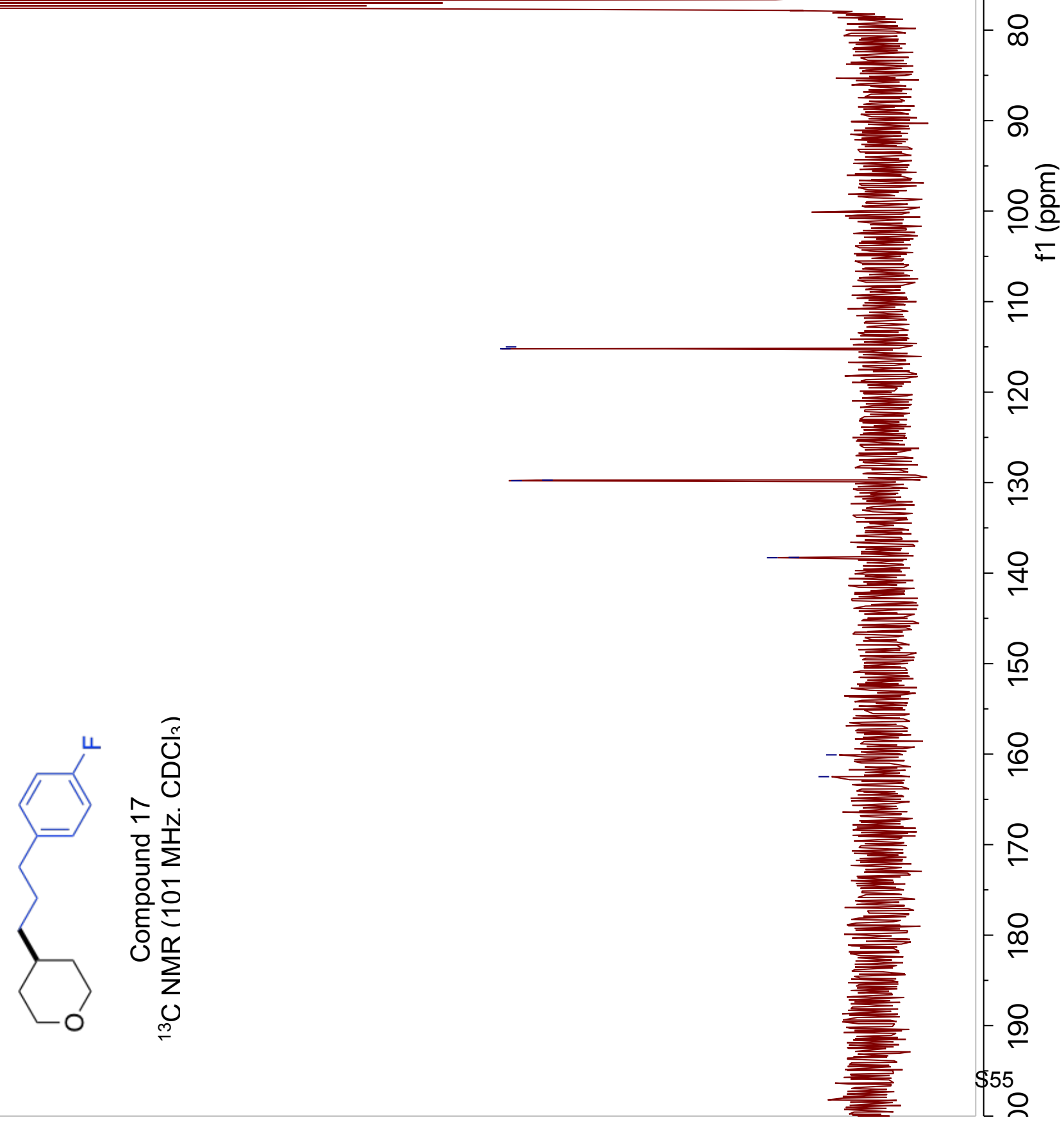


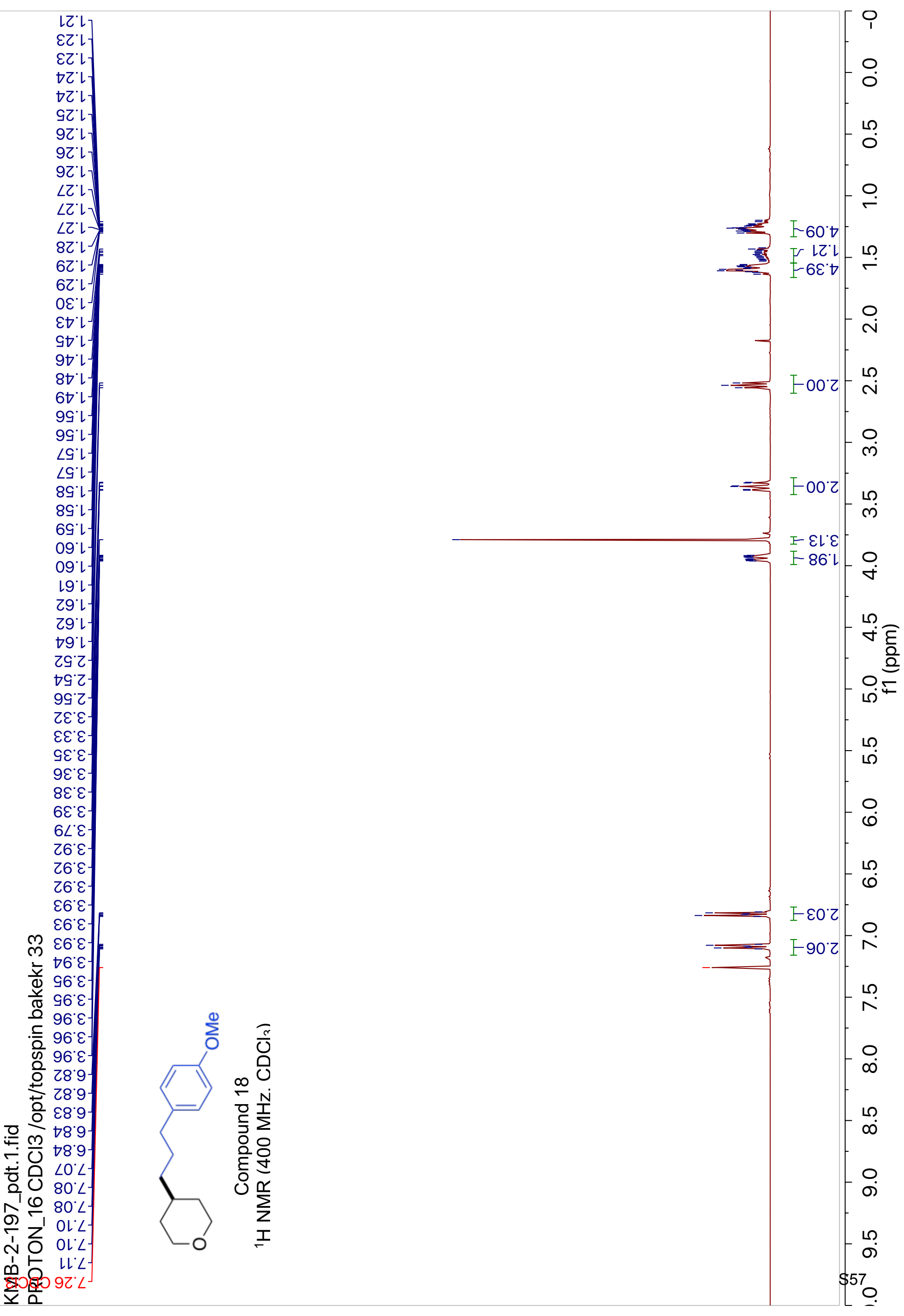


6982

$0 \varepsilon^{\circ} \varepsilon \varepsilon$

ง० $9 \varepsilon$ ᄂ

$6 \tau^{\circ} 9 \varepsilon$

99.9 $9 \varepsilon^{\top}$

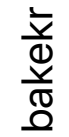
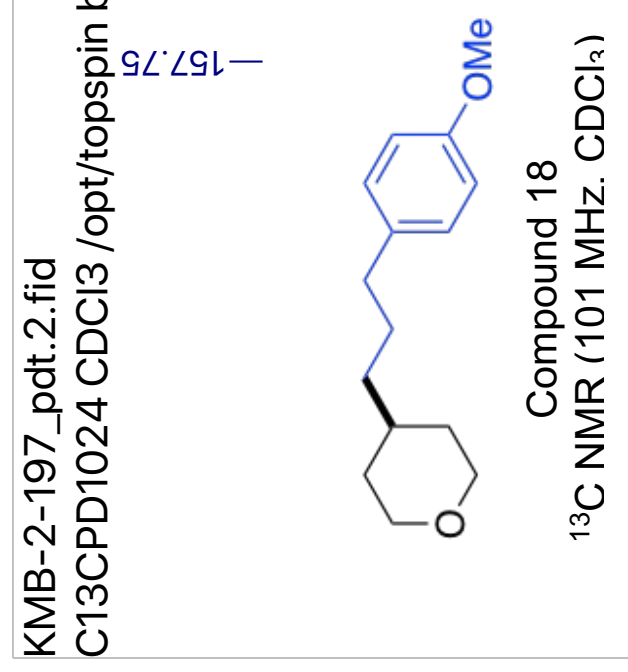


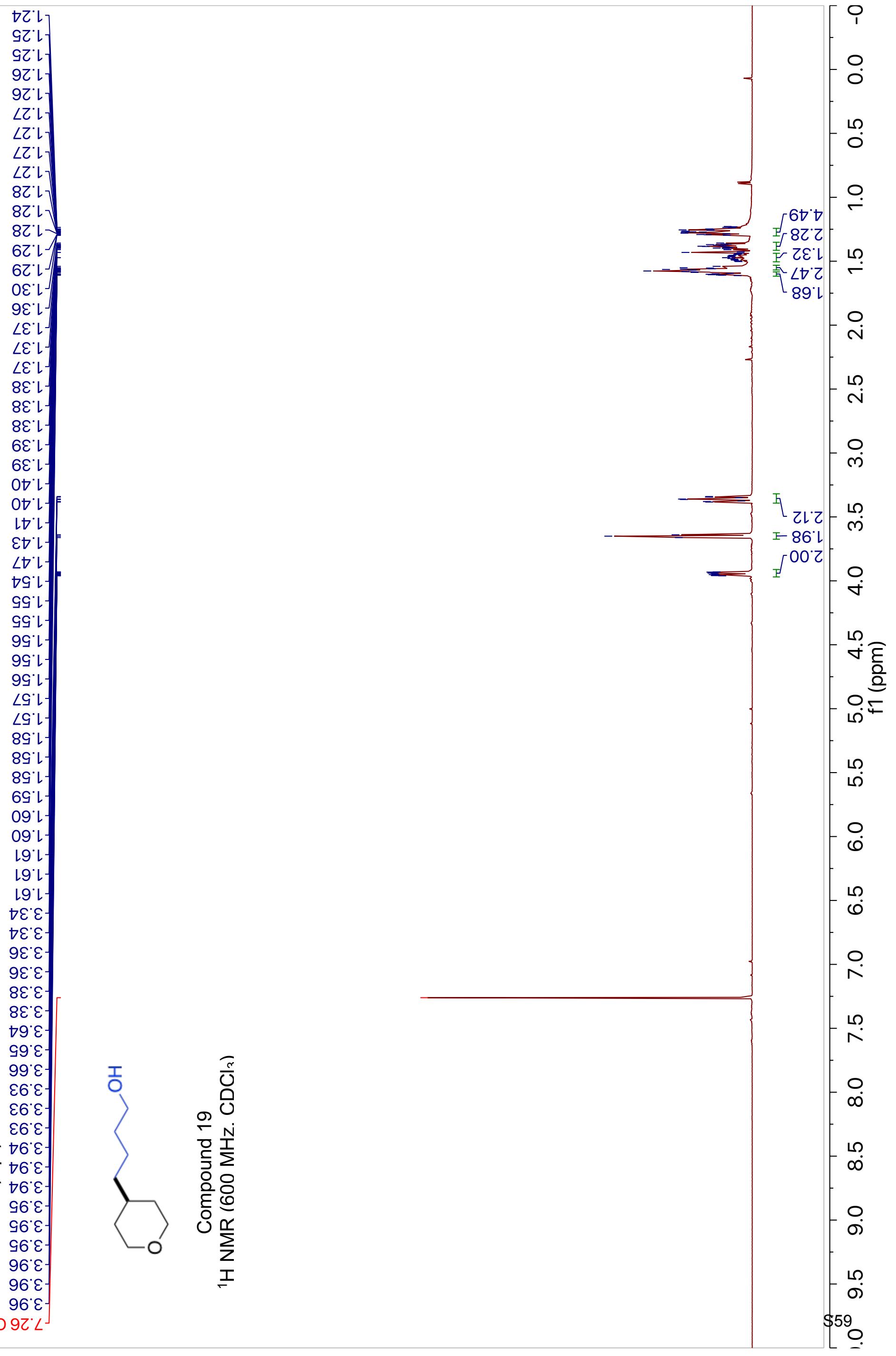


69'2Z-

$\left.\varepsilon 0^{\circ} \varepsilon \varepsilon\right]$

$6 \tau^{\circ} \varepsilon \varepsilon-$

$60.9 \varepsilon$

$98 \varepsilon^{\circ}$

OL'E9-

0ะ.89-

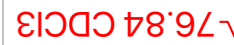

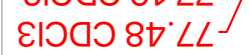

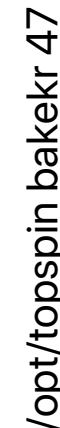

은 m

ํํㅇ

ํำ

유으

ㄴ.

$\sum_{\Sigma}^{\infty} \frac{m}{\circlearrowright}$

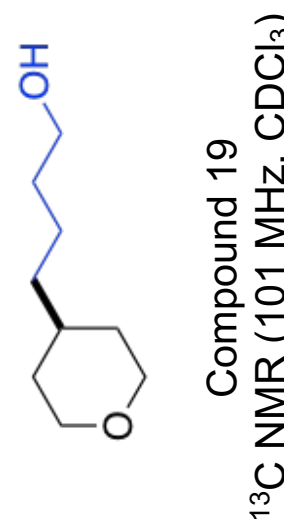

$-\stackrel{8}{\circ}$

$\stackrel{ }{1}$

$\stackrel{\infty}{\stackrel{\infty}{\infty}}$

옴 


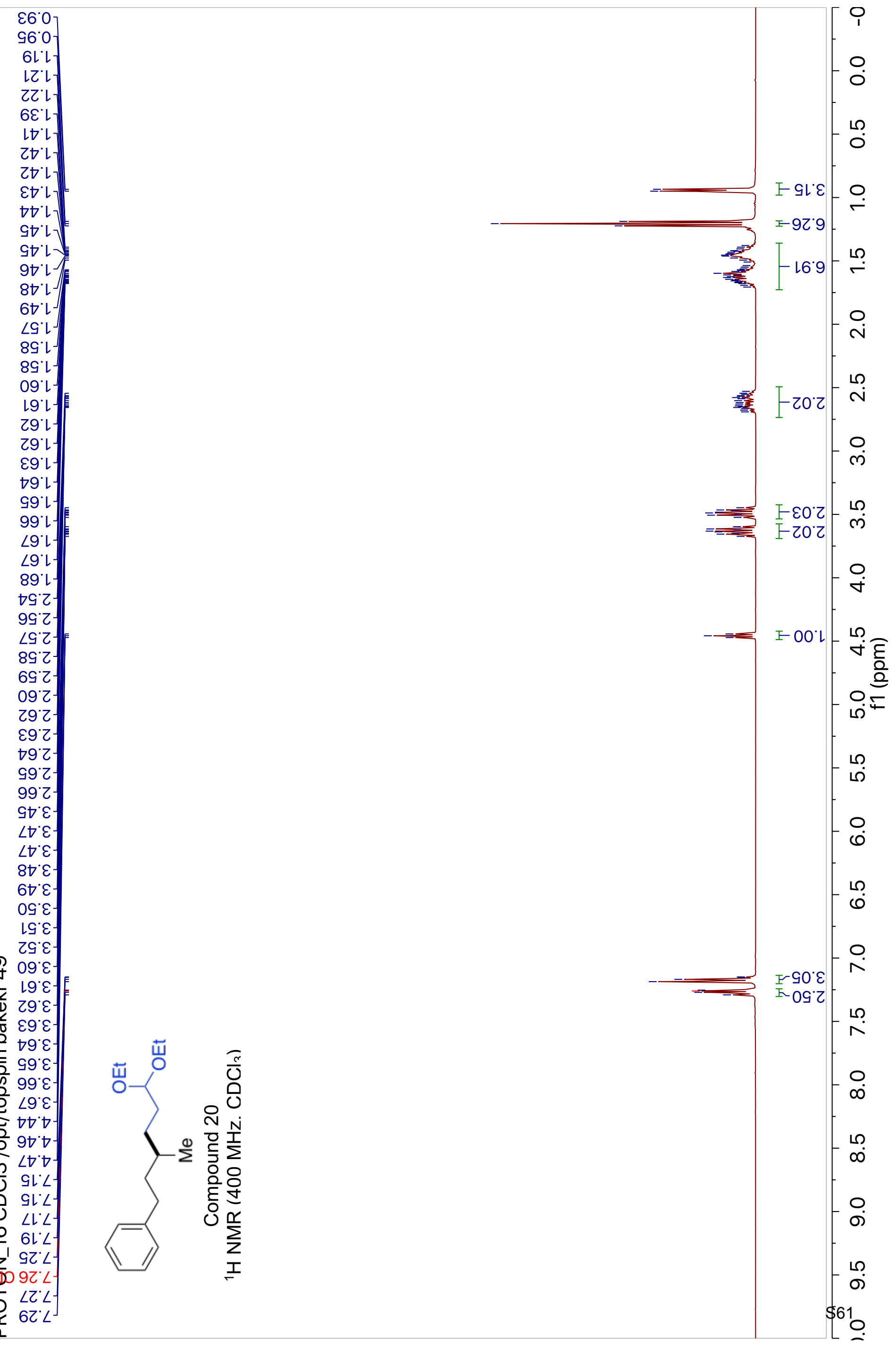


OG'GL-

99.6L-

$\varepsilon L^{\prime} L E$

$\nabla \angle L E-$

$\angle \nabla Z \varepsilon$

G9. $\varepsilon \varepsilon^{\top}$

$\nabla 6 \varepsilon^{\circ}$

으

- 으

- 으

16.097

$\angle 6.09\}$

$-8$

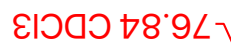

हஒ

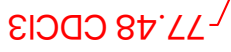

เE'EOL-

69.9Zᄂ

$6 \varepsilon^{\circ} 8 Z L$

$\angle \nabla \cdot 8 Z L$
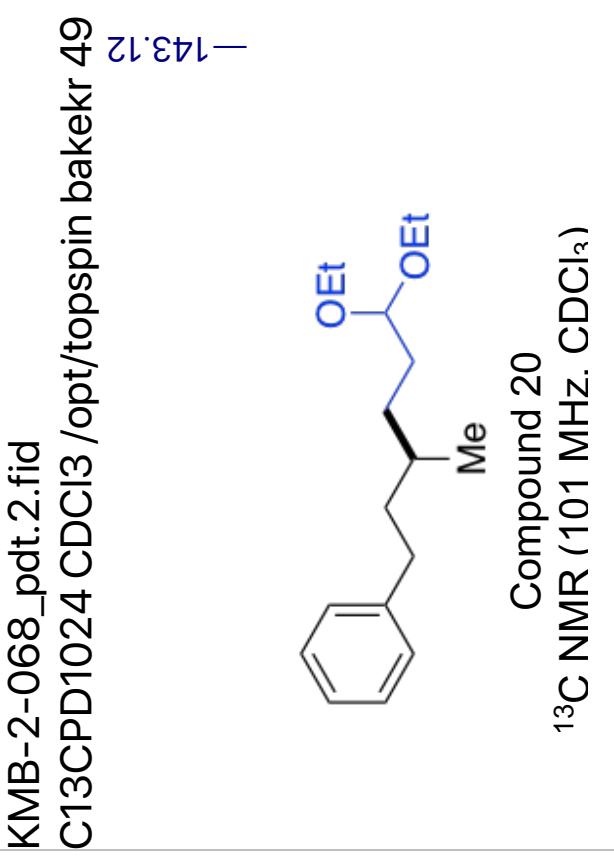

-

$\stackrel{?}{2}$

$\stackrel{\infty}{\stackrel{\infty}{\infty}}$

- 요 


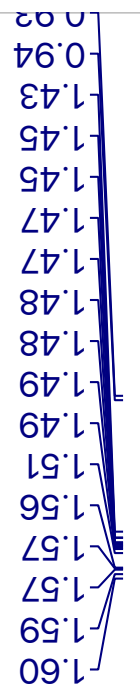

$\varepsilon 9^{\circ} 乙$

G9'乙

99'乙

99'

$\angle G^{\circ}{ }^{\prime}$

$98^{\circ} \varepsilon$

$88^{\circ} \varepsilon^{-}$

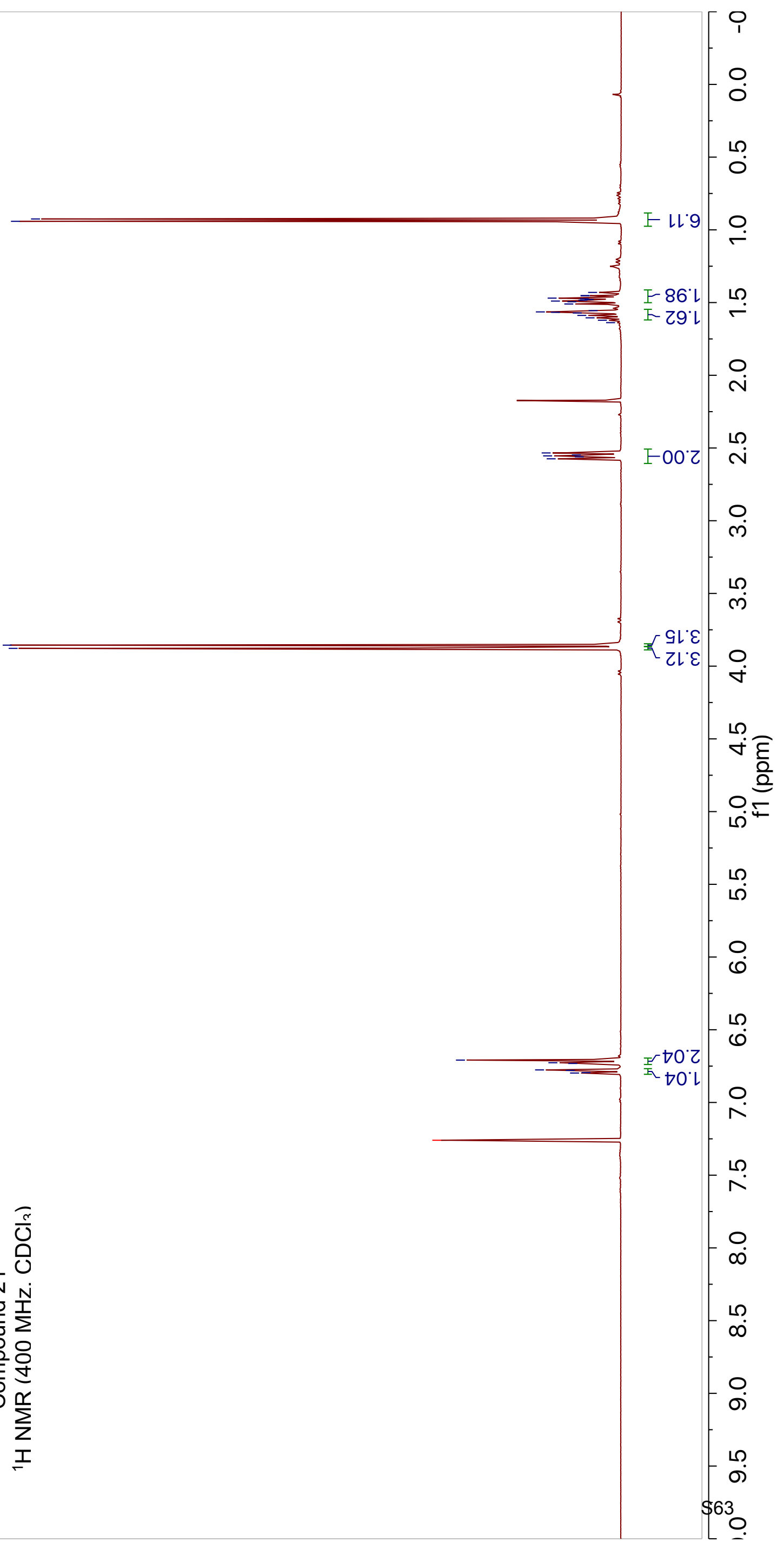




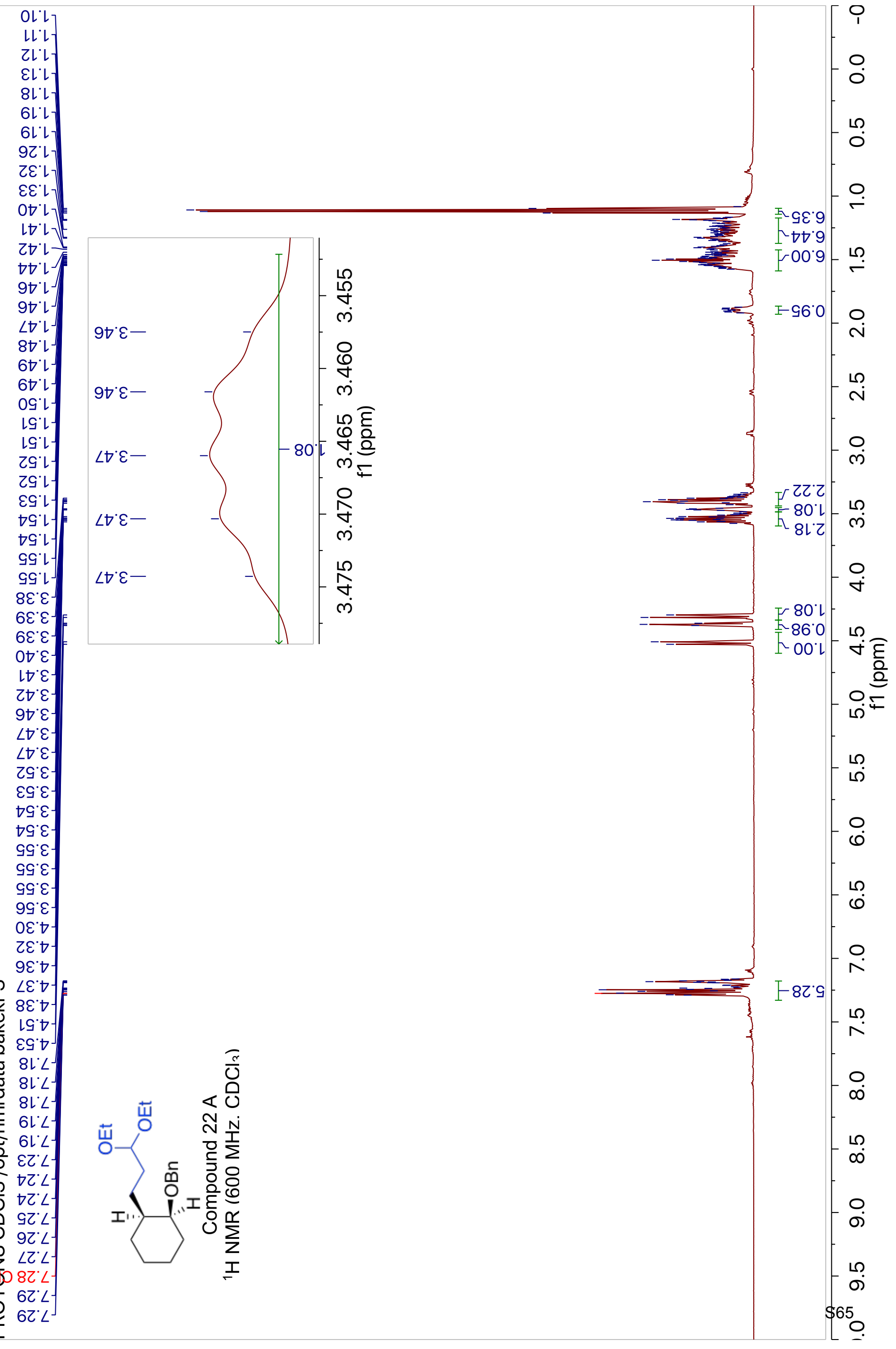


$\angle E^{\circ} G L$

ULLZ

$86^{\circ} \circ Z$

$\left.9 t^{\circ} \angle Z\right\rceil$

09.82 ]

$86^{\circ} 0 \varepsilon$

$9 Z^{\circ}\left\llcorner\varepsilon^{-}\right.$

२৪०0ヤ-

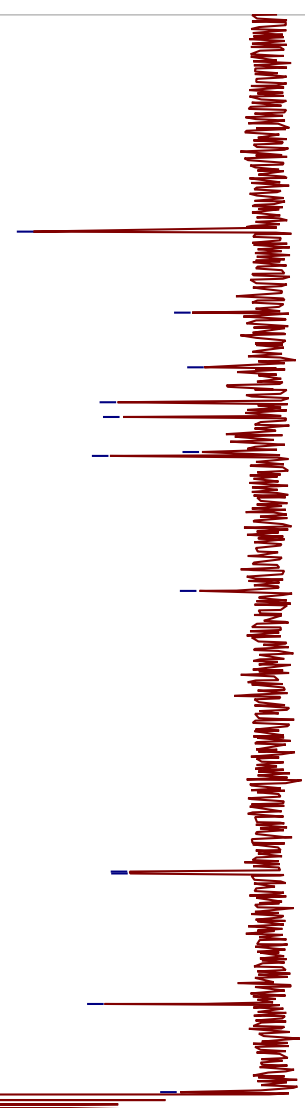

$80.0 \angle-$

$\varepsilon \varepsilon^{\circ} 9 /$

عl200/.9/

हागดว EO LLJ

हाวดว $9 \varepsilon^{\circ} \angle L$

97'عOL-

$8 l^{\circ} \angle Z L$
$Z \nabla^{\circ} \angle Z L$
$6 l^{\circ} 8 Z L$

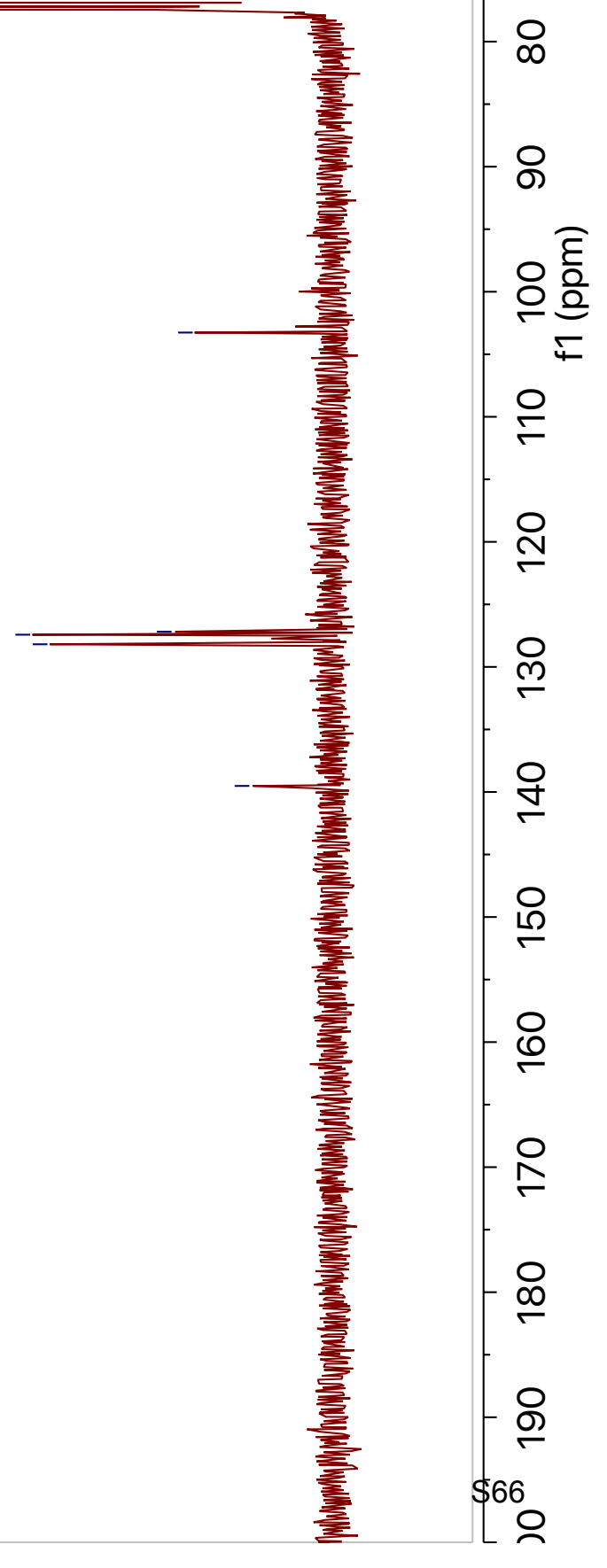

Z9.6el-

N

产

ত্

등

믄 $\frac{m}{0}$

ำ

'ं 0

ป พ

ำ

ㅇำ

$\sum_{\Sigma}^{\infty} \frac{\mathrm{m}}{\mathrm{u}}$

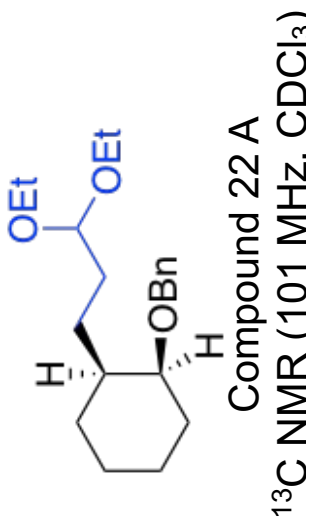

ㅇํำ

8



$\infty$

৪

응ㅎㅇ

N

으

옹

8

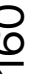

$\underline{2}$

$\stackrel{\infty}{\infty}$

용 


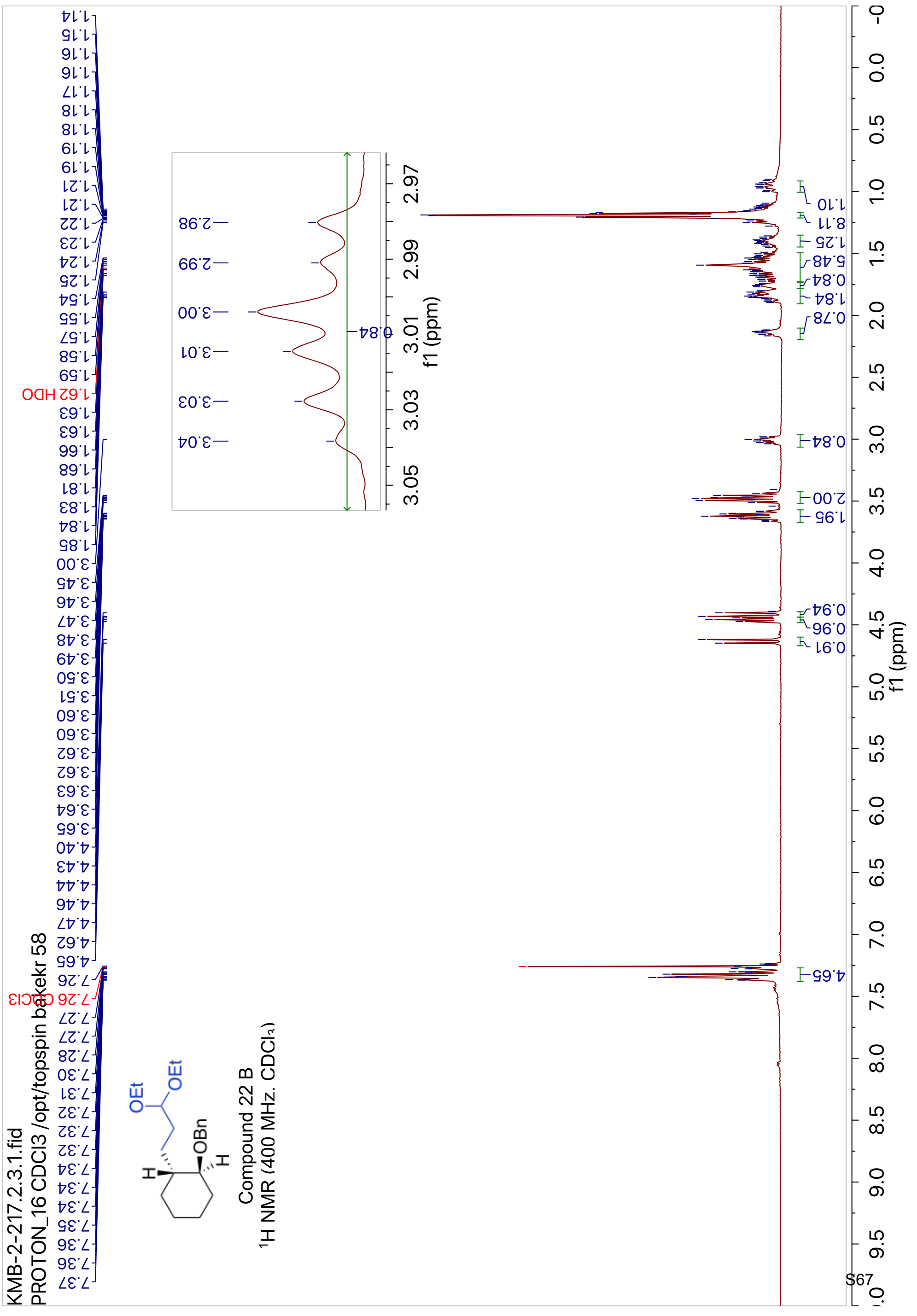


$\left.\begin{array}{l}\left\langle\varepsilon^{\circ} \text { Gl }^{\prime}\right. \\ 6 \varepsilon^{\circ} \text { gl }\end{array}\right\rangle$

$L\llcorner\nabla Z$

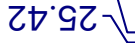

$O Z \angle Z=$

$\varepsilon \varepsilon^{\circ} 0 \varepsilon$

ट9.0ह-

$80^{\circ} L$

68`てー

$09^{\circ} 0 \angle-$

6ะ'عOL-

$\angle \varepsilon^{\prime} \angle Z L$
$9 L \angle Z L$
$\angle Z \cdot 8 Z L$

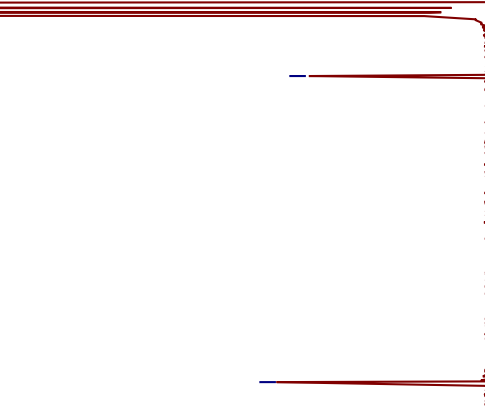

$-\infty$

우

을

을

ํำ

요

g

$\stackrel{\infty}{\infty}$

$\varepsilon l^{\circ} 6 \varepsilon l-$

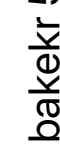

등

은 $\frac{m}{0}$

กับ

ำ

ㄷํㅇ

ำ

궁

$\frac{\omega}{\frac{\omega}{2}} \frac{\underline{0}}{0}$

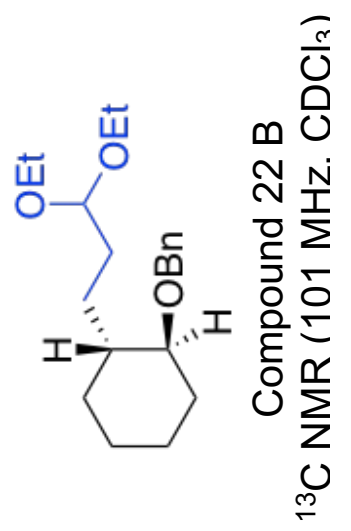

욤

$๕$

$\stackrel{8}{2}$

$\stackrel{\circ}{\check{0}}$

옴 


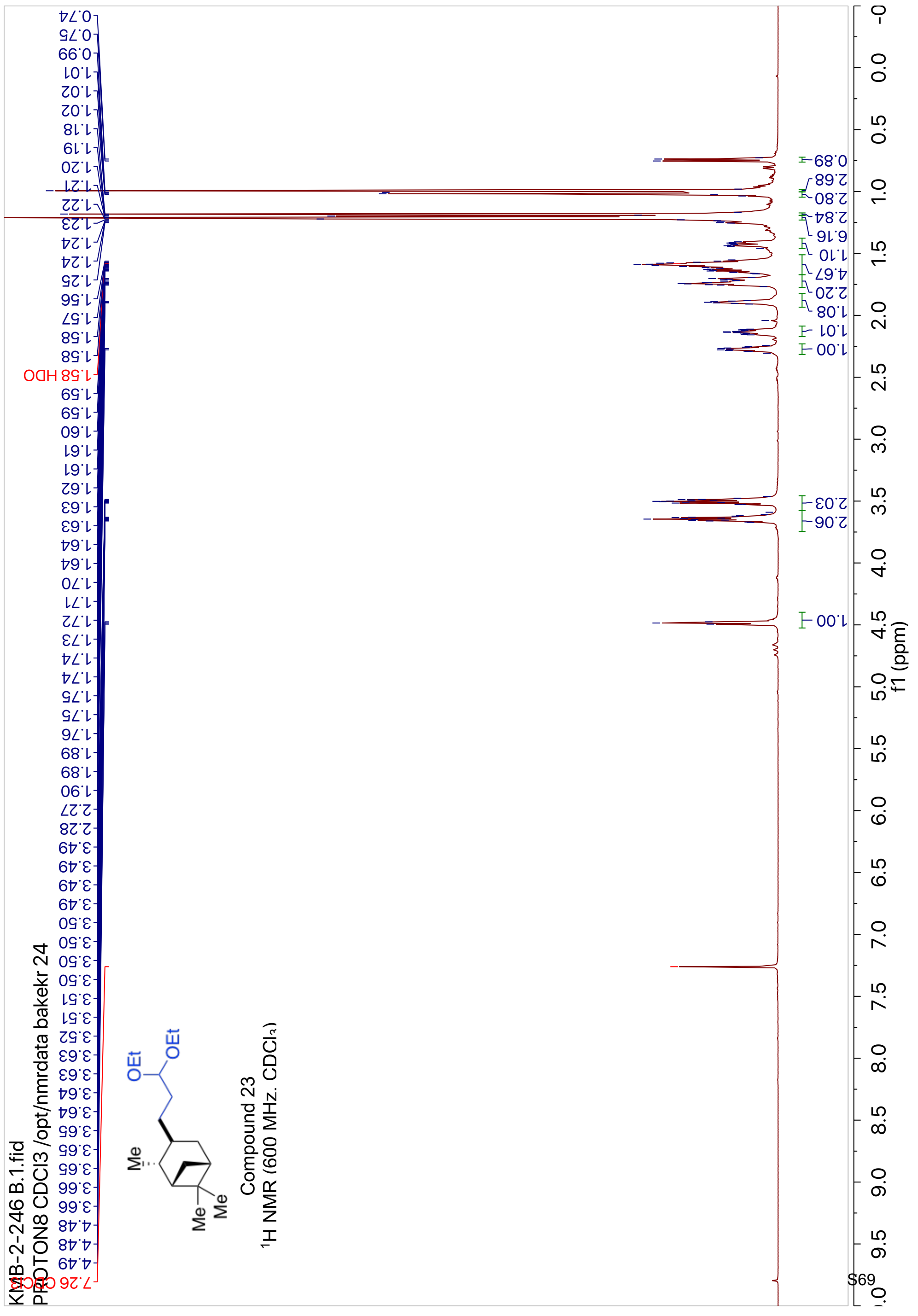


○ย๋レ

EG'GL-

88.12

乙L'ย乙二

$\nabla Z^{\circ} 8 Z$

$\nabla 6 \cdot \varepsilon$

$\angle l^{\circ} \nabla \varepsilon$ ]

$\varepsilon 8^{\circ} \nabla \varepsilon-$

$80^{\circ} 9 \varepsilon-\tau$

$\nabla \nabla^{\circ} 9 \varepsilon$

$16.8 \varepsilon$

$90^{\circ} \mathrm{Z}$

$88^{\circ} \varepsilon \nabla$

$\nabla \varepsilon^{\circ} 8 \nabla^{\top}$

$08^{\circ} 09$

10.19

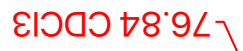
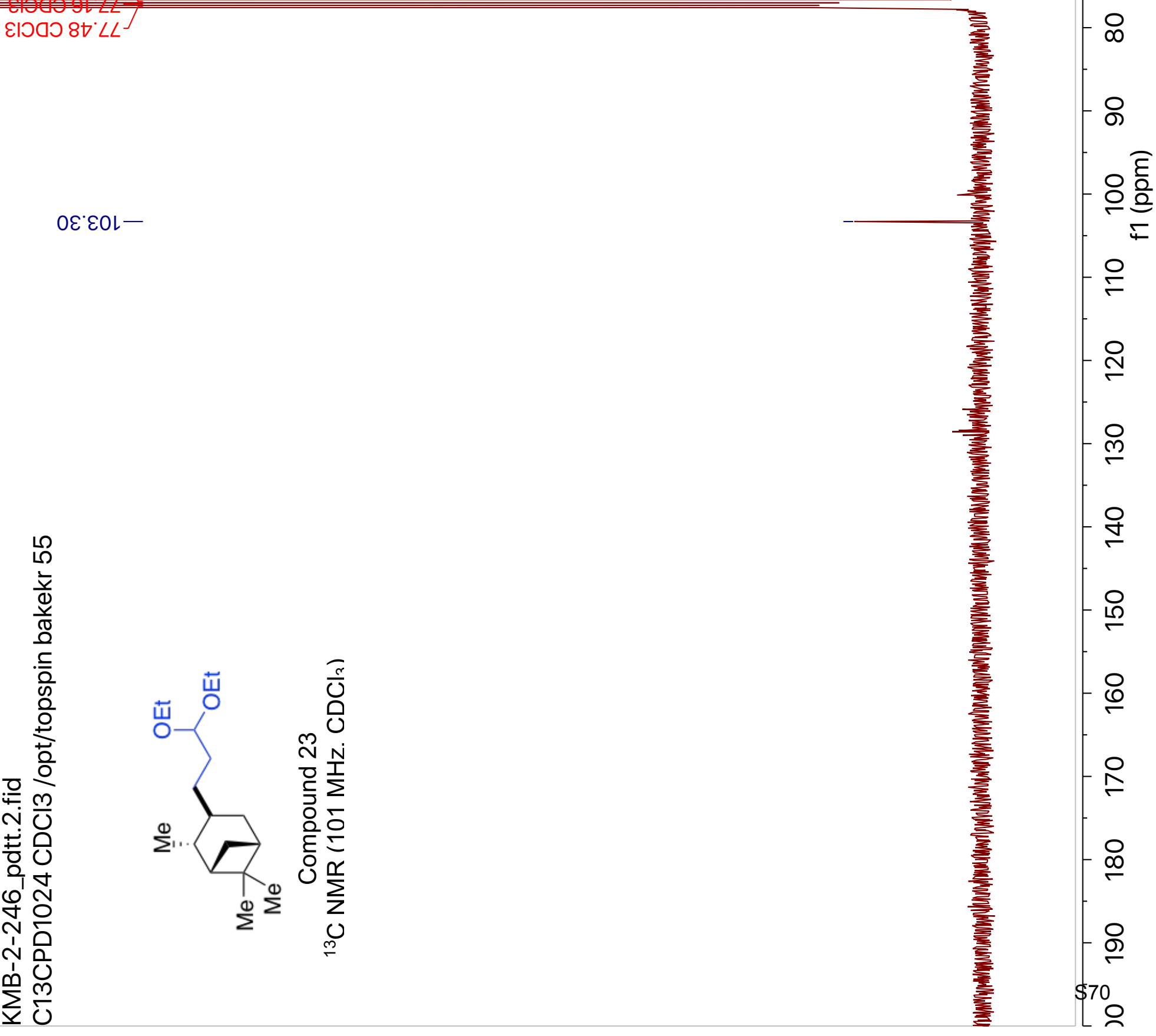


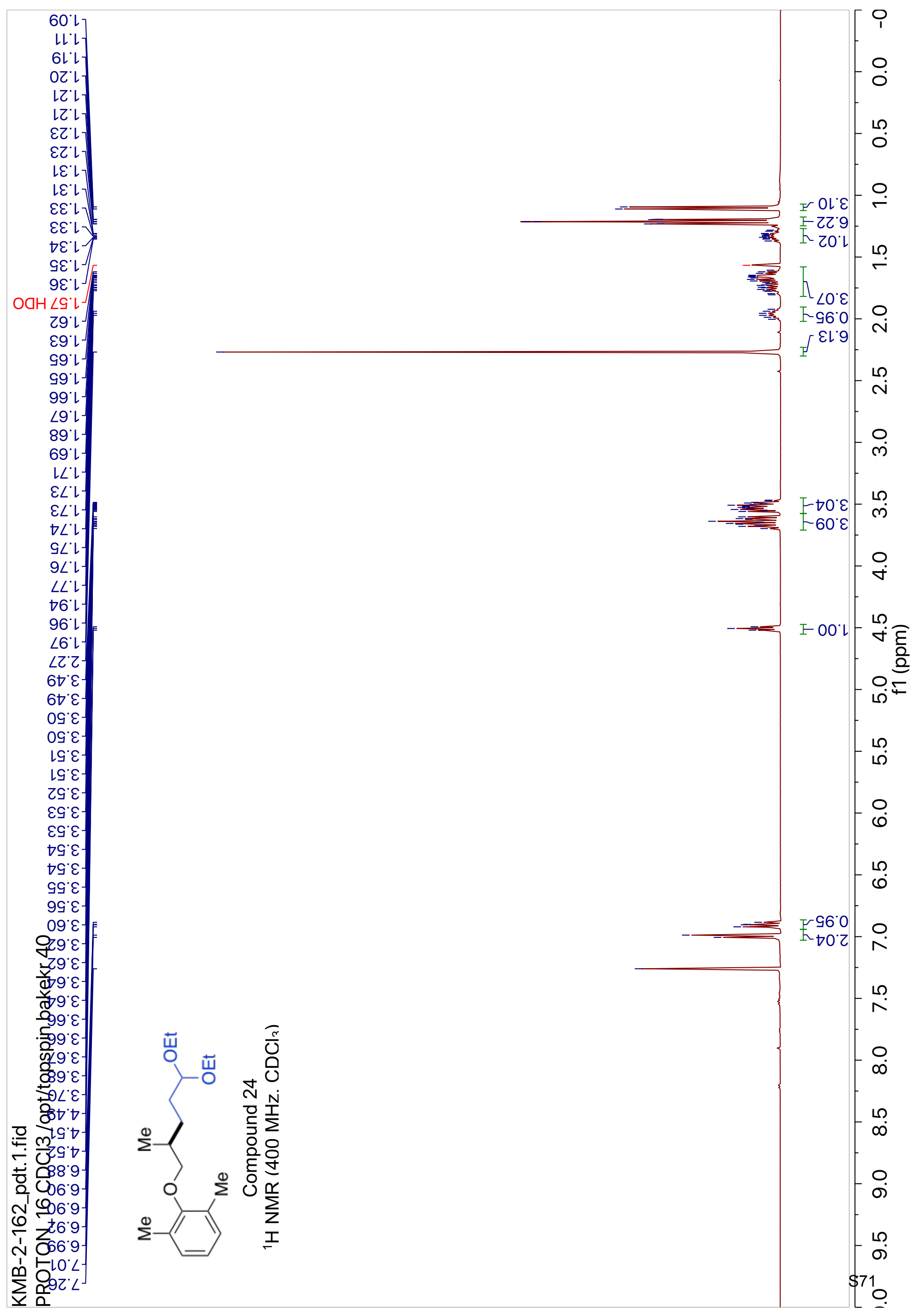




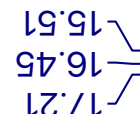

$89.82-$

$6 \varepsilon^{\circ}\llcorner\varepsilon-$

$9 \varepsilon^{\circ} \nabla \varepsilon-$

$\left.\begin{array}{l}60.19 \\ \angle L .19\end{array}\right\rangle$

$-8$

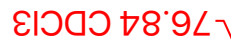

- 웅

हリ

$-\infty$

- 응

oร'EOL-

GL'EZL-

Z6.8ZL

$80^{\circ} \mathrm{LEL}-$

운

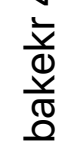

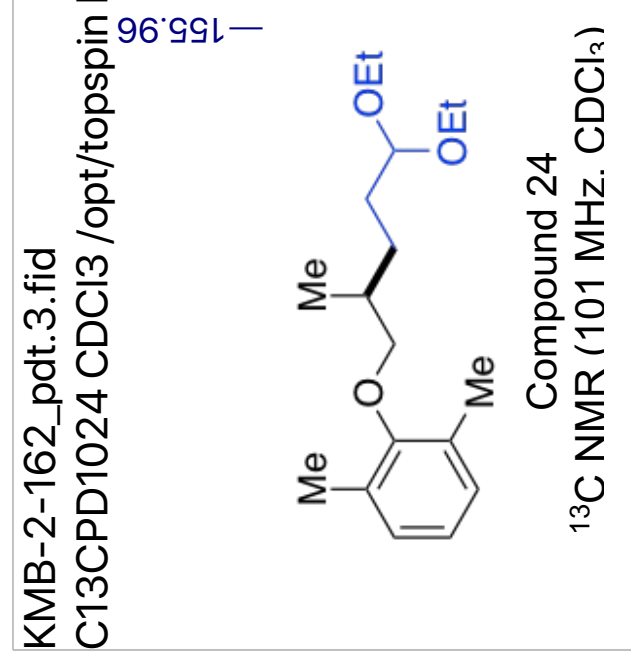

- 음

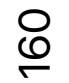

$\stackrel{?}{\square}$

$\stackrel{\infty}{\check{\infty}}$

욤 


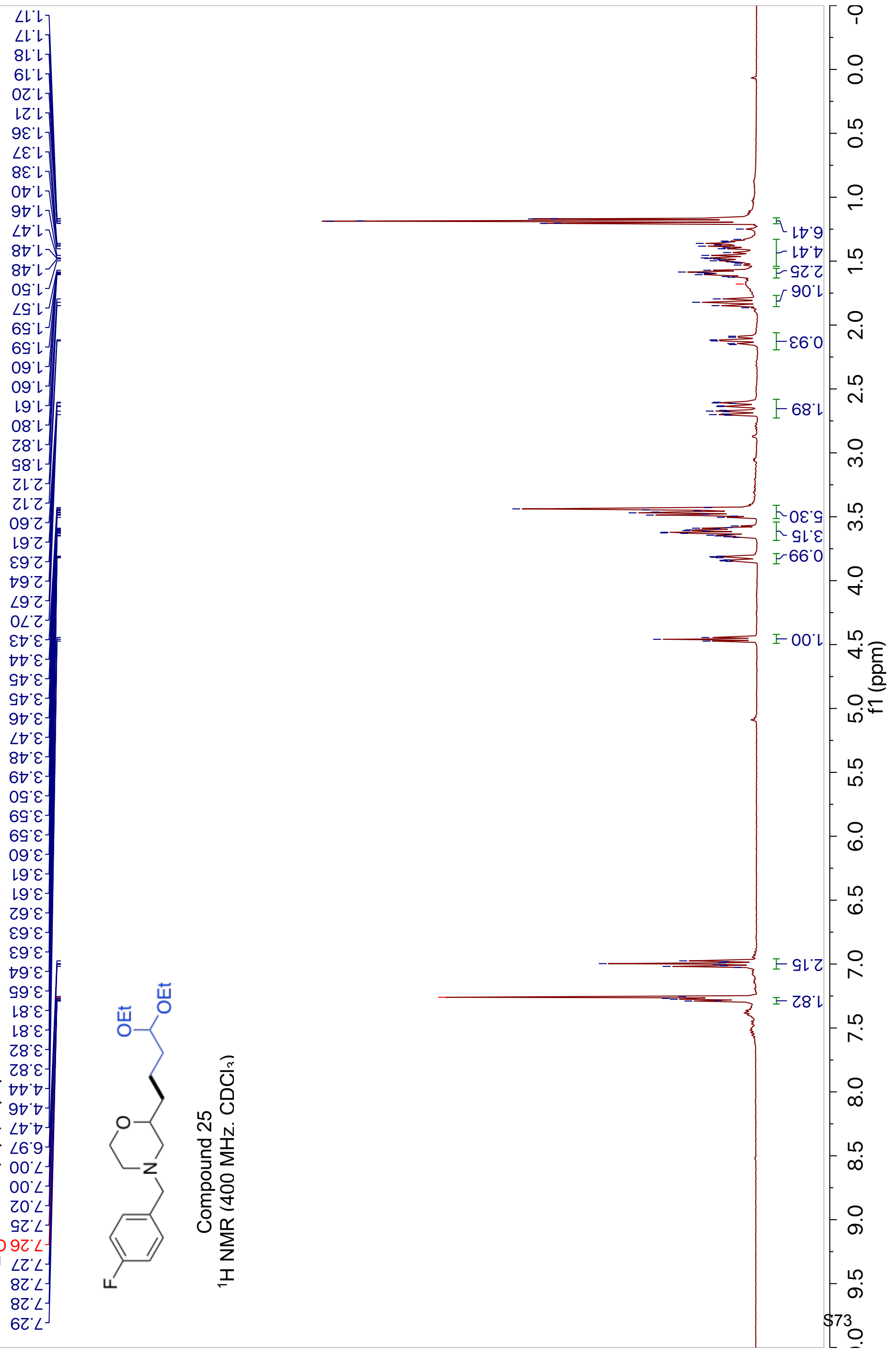


8tง

$18.02-$

$\left.\begin{array}{l}09^{\circ} \varepsilon \varepsilon \\ L{ }^{\circ} \varepsilon \varepsilon\end{array}\right\rangle$

OZ'ยG -

$\varepsilon \angle \cdot 89$

96.09

9l.19-

09.29

$\angle 8.99$ -

$L \angle \cdot G L$

हા๐๐ $\nabla 8^{\circ} 9 \angle \backslash$

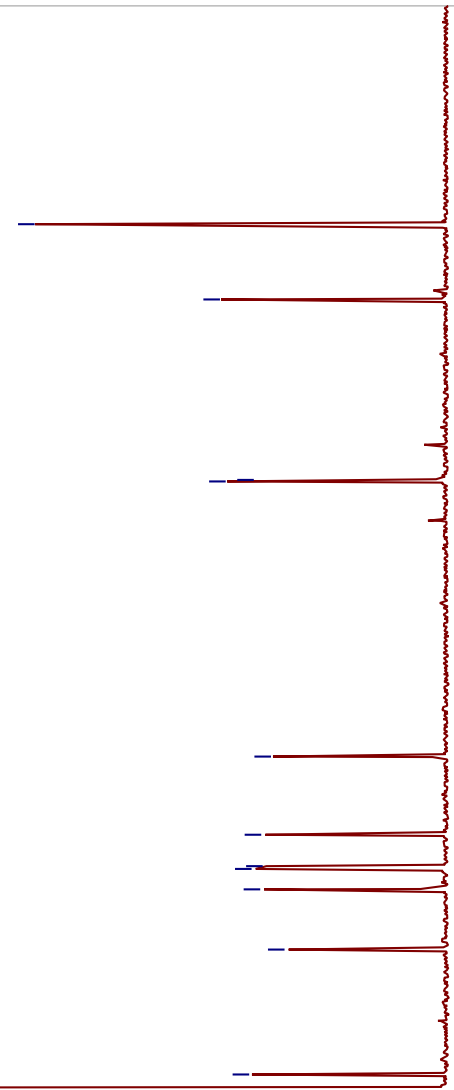

$\stackrel{ }{\circ}$

$\stackrel{ }{\circ}$

ल

$\stackrel{\bigcirc}{\checkmark}$

웅

8

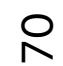

हागUJ 9L LL]

हІวดว 8t $\angle L$

06 ZOL-

60'9lᄂ

OE'GLᄂ

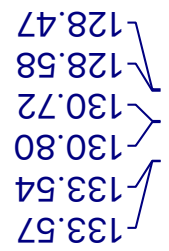

กิ

$\frac{\bar{v}}{0}$
$\frac{1}{0}$
$\frac{1}{2}$

등

ตำ6.09L-

을 $\angle \varepsilon^{\circ} \varepsilon 9 l-$

응

은 $\frac{\mathrm{m}}{\mathrm{d}}$

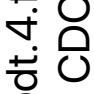

สำ

두음

ㄴํㅁ

$\sum_{i}^{\prime} \frac{m}{\sigma}$

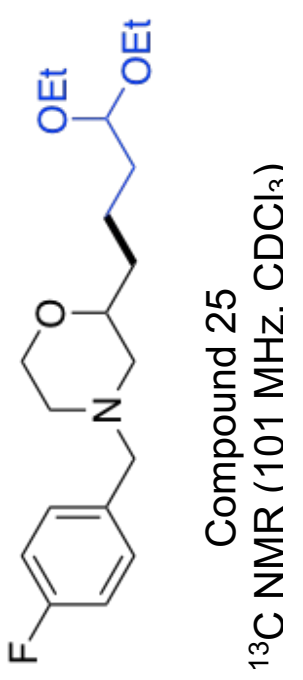

$-\infty$

ㅇ

을

을

ํำ

$\stackrel{ }{\stackrel{9}{2}}$

g

$\stackrel{\circ}{\circ}$

$\stackrel{8}{0}$

$\stackrel{ }{\stackrel{2}{2}}$

$\stackrel{\circ}{\stackrel{0}{ }}$

옴 


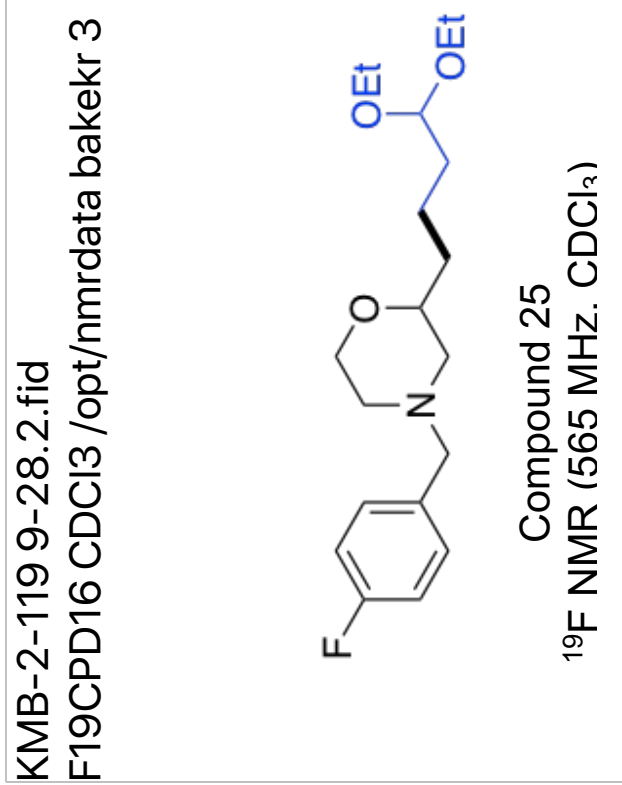




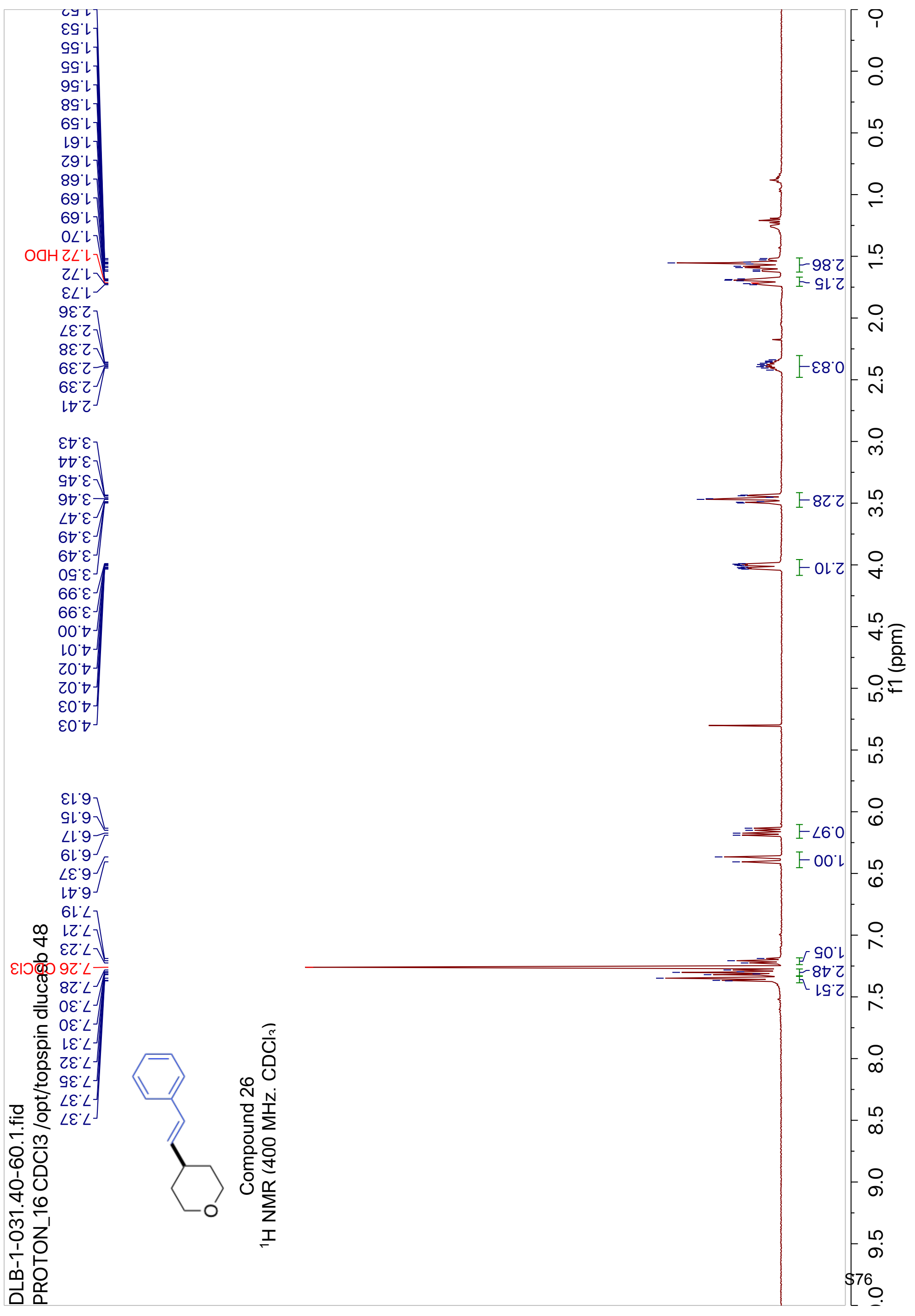




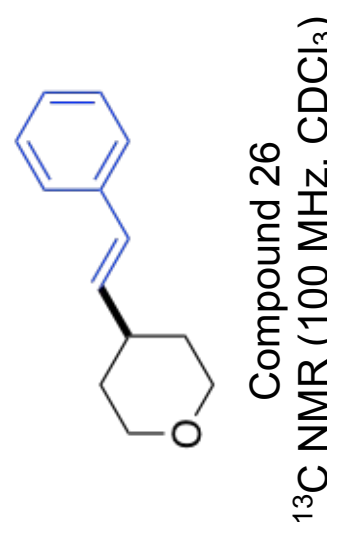




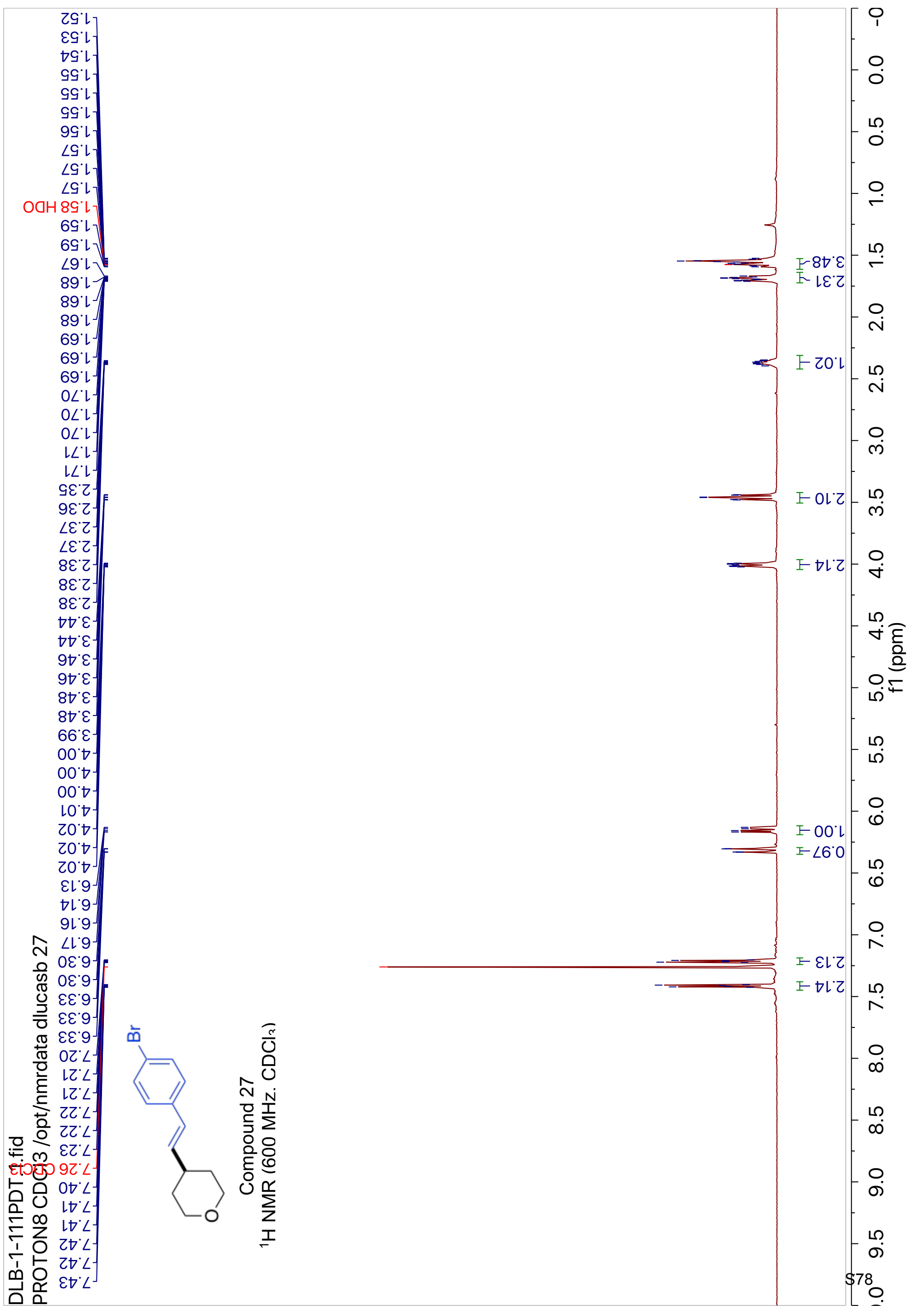


69 'น-

$\varepsilon \mathcal{c}^{\circ} 8 \varepsilon-$

$\varepsilon 8: \angle 9-$

ह1วดว $96^{\circ} 9 /$

EाOOO $9 l^{\circ}<L J$

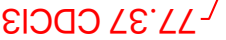

06.0ZL-

$8 \varepsilon^{\circ} \angle Z L$

$9 \angle \angle Z L J$

GL'LEL-

09.9EL

69.9عا

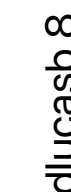

$\infty$
용
$\frac{0}{0}$
$\frac{0}{0}$

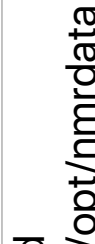

은 $\mathrm{m}$

กำ

เิ

$\infty \underset{\sim}{\infty}$

둥 음

宁吕

เ

ज)

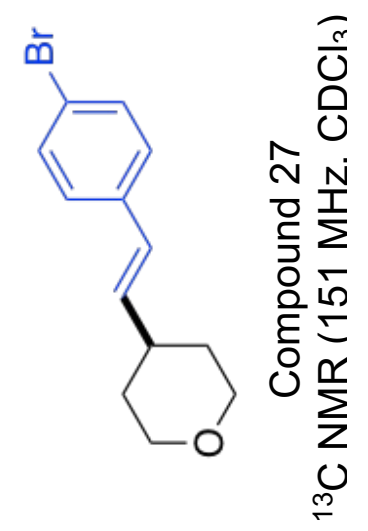




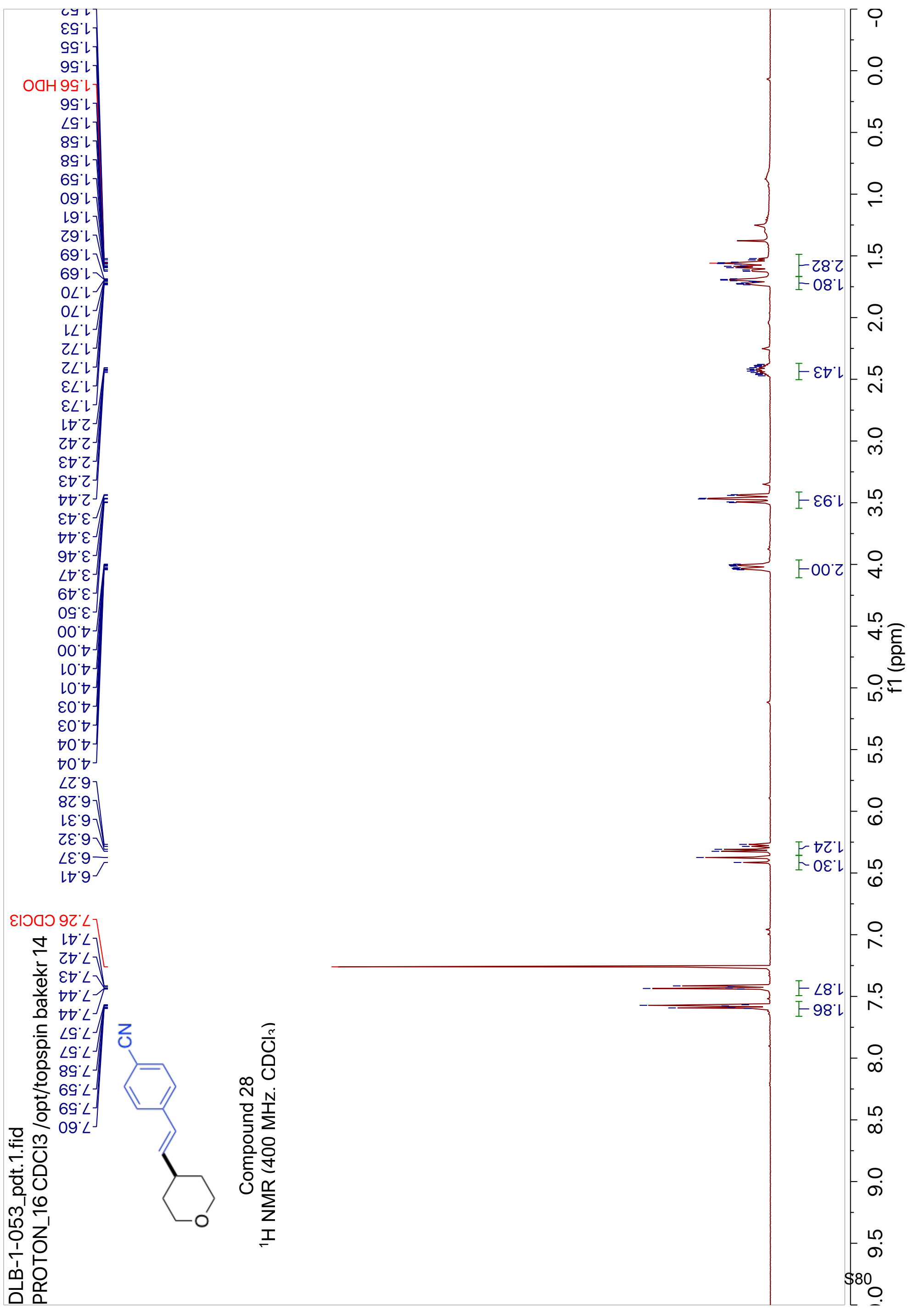


เナてとー

$\triangleright 9^{\circ} 8 \varepsilon-$

عで6Lー

$\angle 9^{\circ} 9 Z L T$
ll $\angle Z L$

乙G'てहь-

08.8EL-

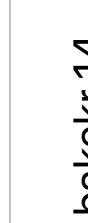

8レてヤーー

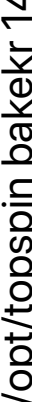

은 $\mathrm{m}$

त

ㄷํㅇ

(1)

กำ ำ

ㅇำ

宁

$m$

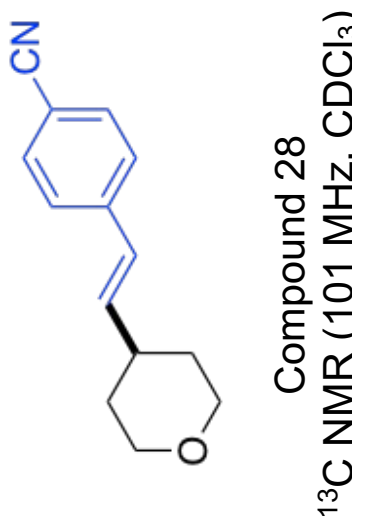

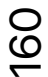

$\stackrel{ }{\stackrel{2}{2}}$

$\stackrel{\infty}{\stackrel{\infty}{\infty}}$ 


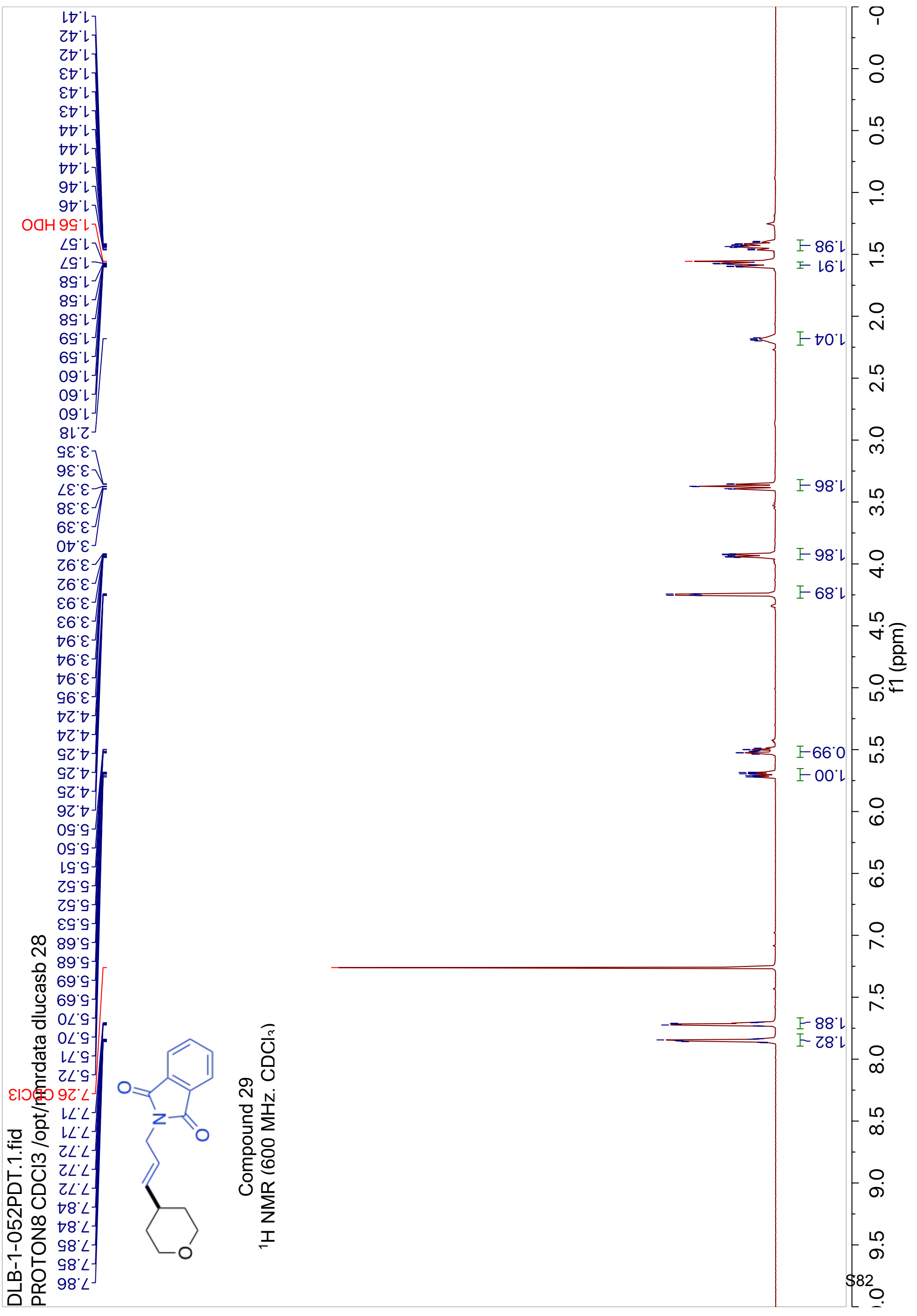


L6.LL

6ย'ย乙レ-

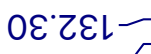

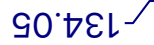

\&6.8عL -

$\hat{0}$
$\frac{0}{\omega}$
J్
$\frac{\partial}{0}$

〈>

ต

D̄

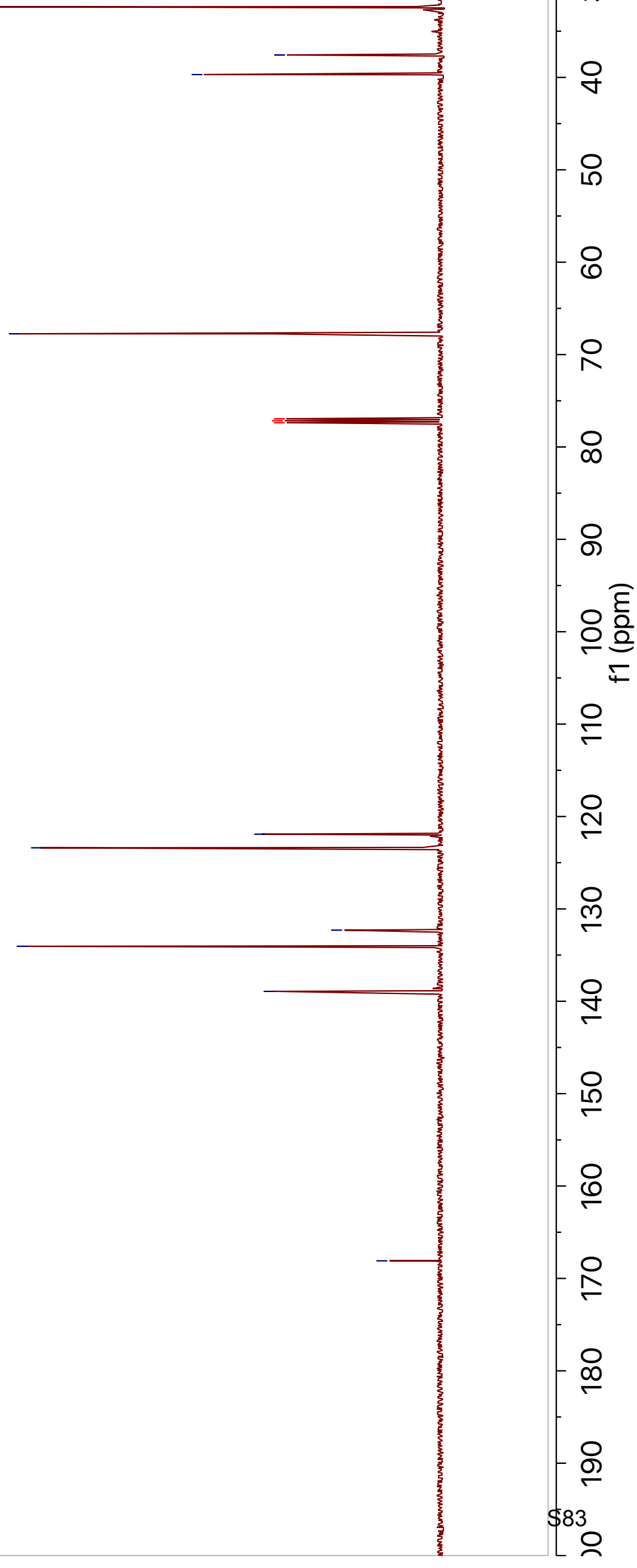




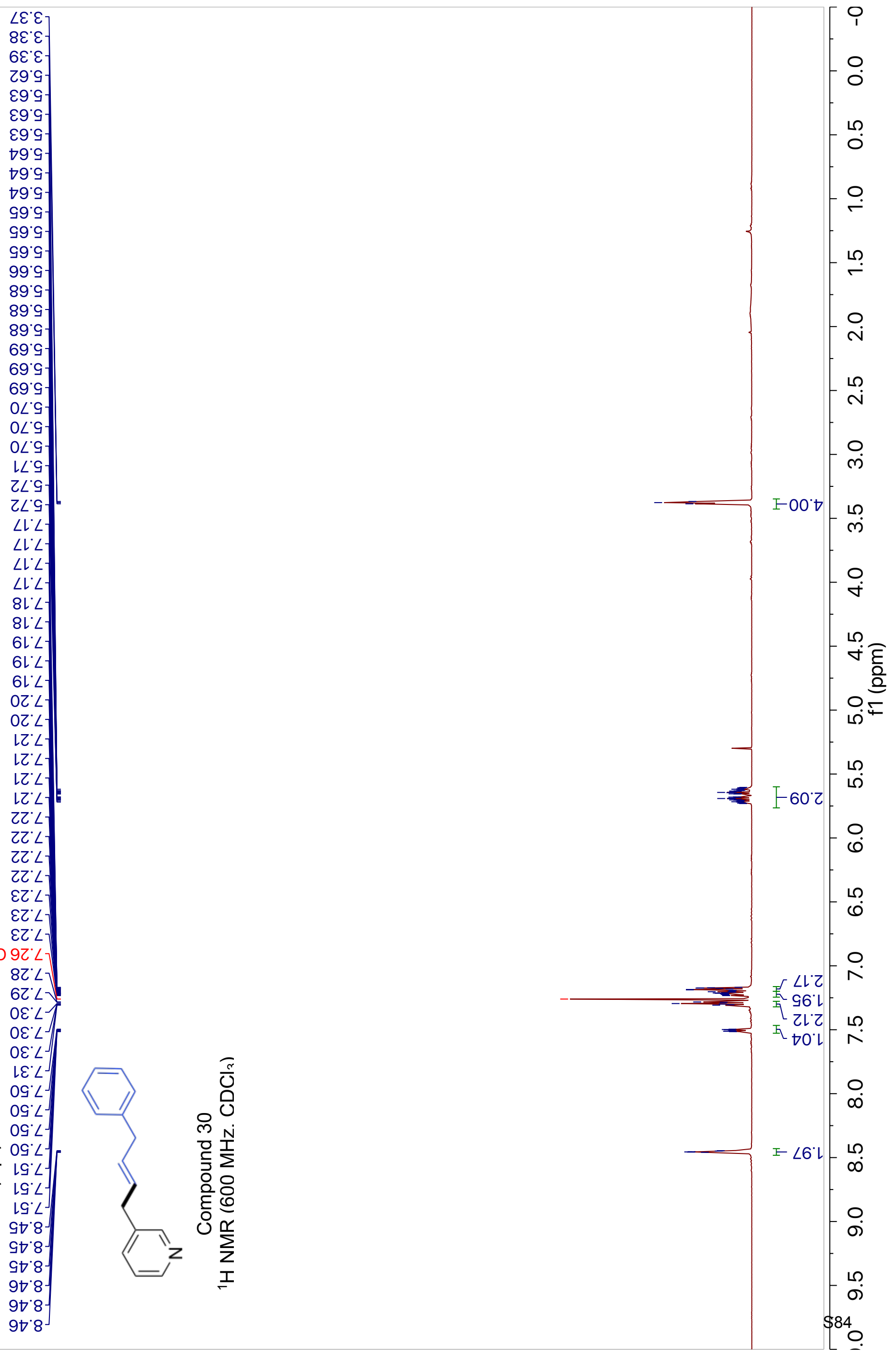


$\angle L \cdot 9 \varepsilon-$

$90{ }^{\circ} 6 \varepsilon-$

हાวดว 96.9L हા๐०ว $9 l^{\circ} \angle L \div$

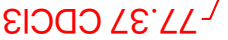

OG'๕ZL

$\varepsilon Z^{\prime} 9 Z L$

$09.8 Z L$

$79^{\circ} 8 Z L$

$0 \angle{ }^{\circ} 8 \mathrm{LZ}-$

8l.6Zl

GL'LEL

†L'9عL-

m $97 \cdot 9 \varepsilon 1]_{1}$

으 $\angle T^{\circ} 0 t L$

ठ 89' $\angle \nabla L-$

긍 90.09L-
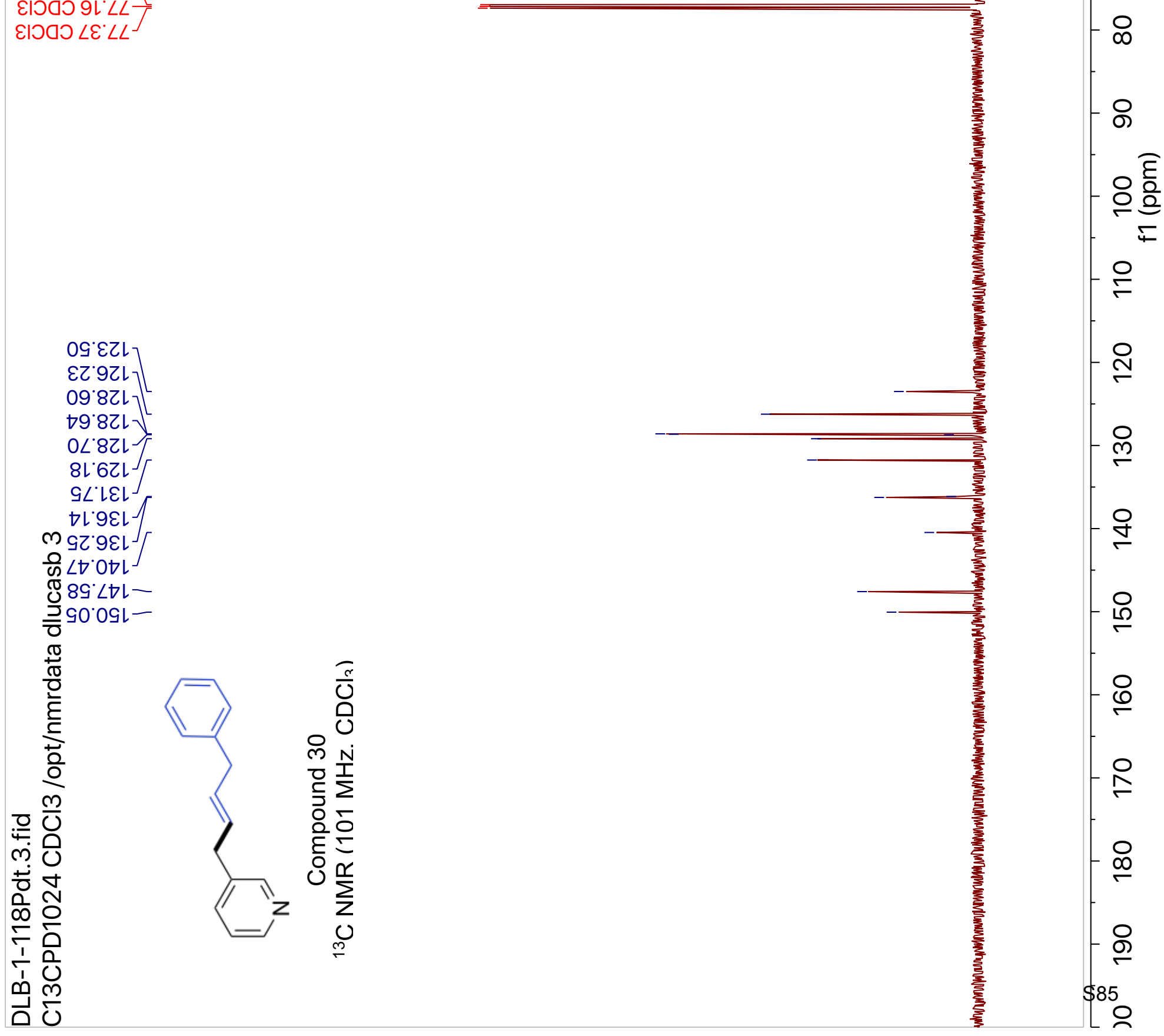


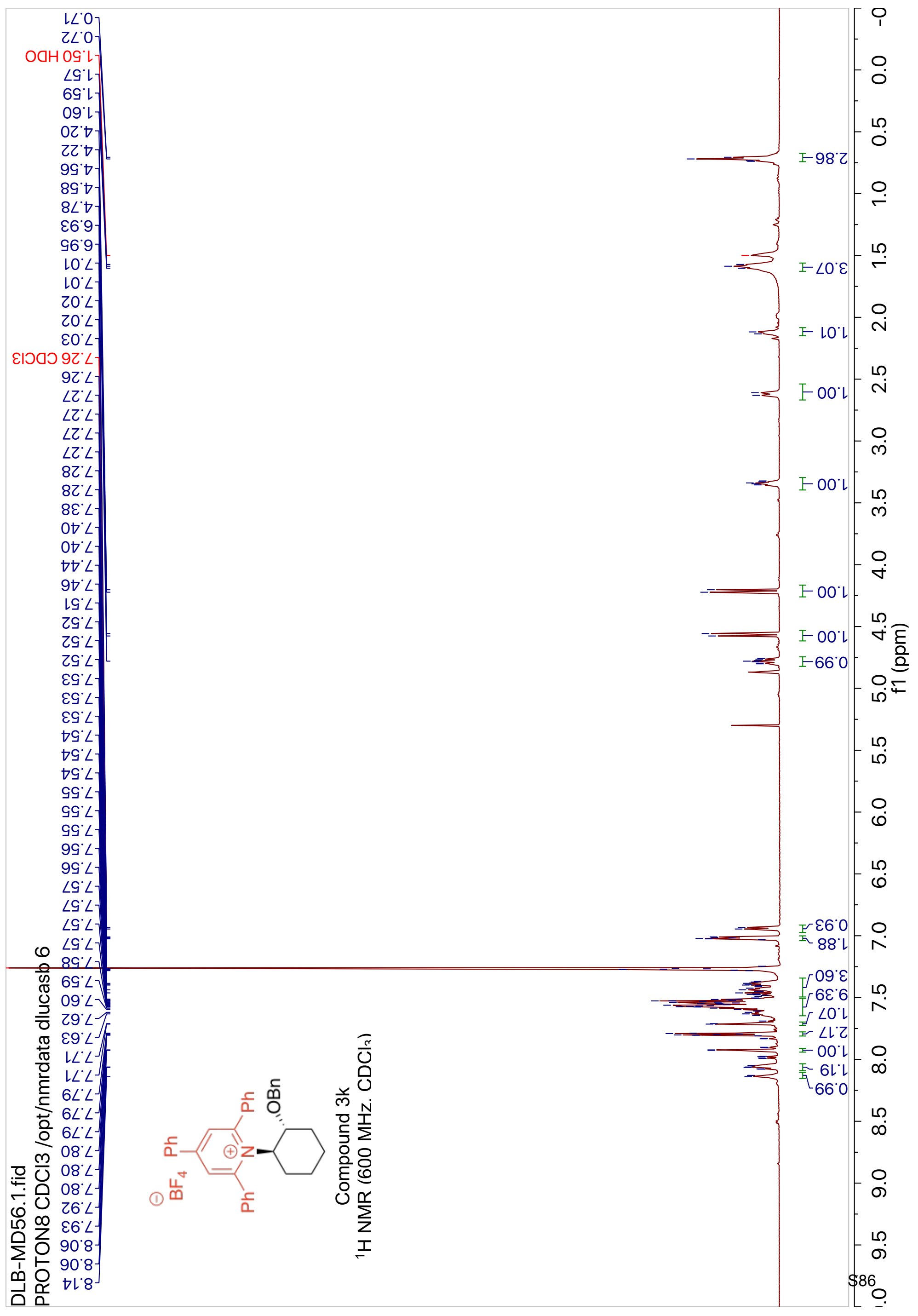


$\downarrow \varepsilon^{\circ} \varepsilon 乙-$

L9.SZ-

$\succ 9^{\circ} \tau \varepsilon$
$\angle \varepsilon^{\circ} Z \varepsilon^{\prime}$

$98^{\circ} 89-$

I $\angle \circ L-$

ES $\angle L-$

乙๐.9ZL

S8.9ZI

I6 $\angle 2$ I -

$56^{\circ} \angle Z \mathrm{I}$

$0 Z^{\circ} 8 \mathrm{LI}$

9t.8ZI

L $\angle{ }^{\circ} 8 Z \mathrm{~L}$ -

ธะ.6ZI

6S.6ZI]

99.6ZI

ZL०OEI T

$66^{\circ} 0 \varepsilon \mathrm{I}$ 乍

โ6 ${ }^{\circ}$ LI I

¿० $ح \varepsilon I-$

ZS $દ \varepsilon I-$

$96^{\circ} \varepsilon \varepsilon \mathrm{I}$

$82^{\circ} \dagger \varepsilon \mathrm{I}$

$00^{\circ} 8 \varepsilon[$

$76^{\circ} \mathrm{tS} \mathrm{I}$

8L'9SI-

ع ${ }^{\circ} 6 \mathrm{SI}-$
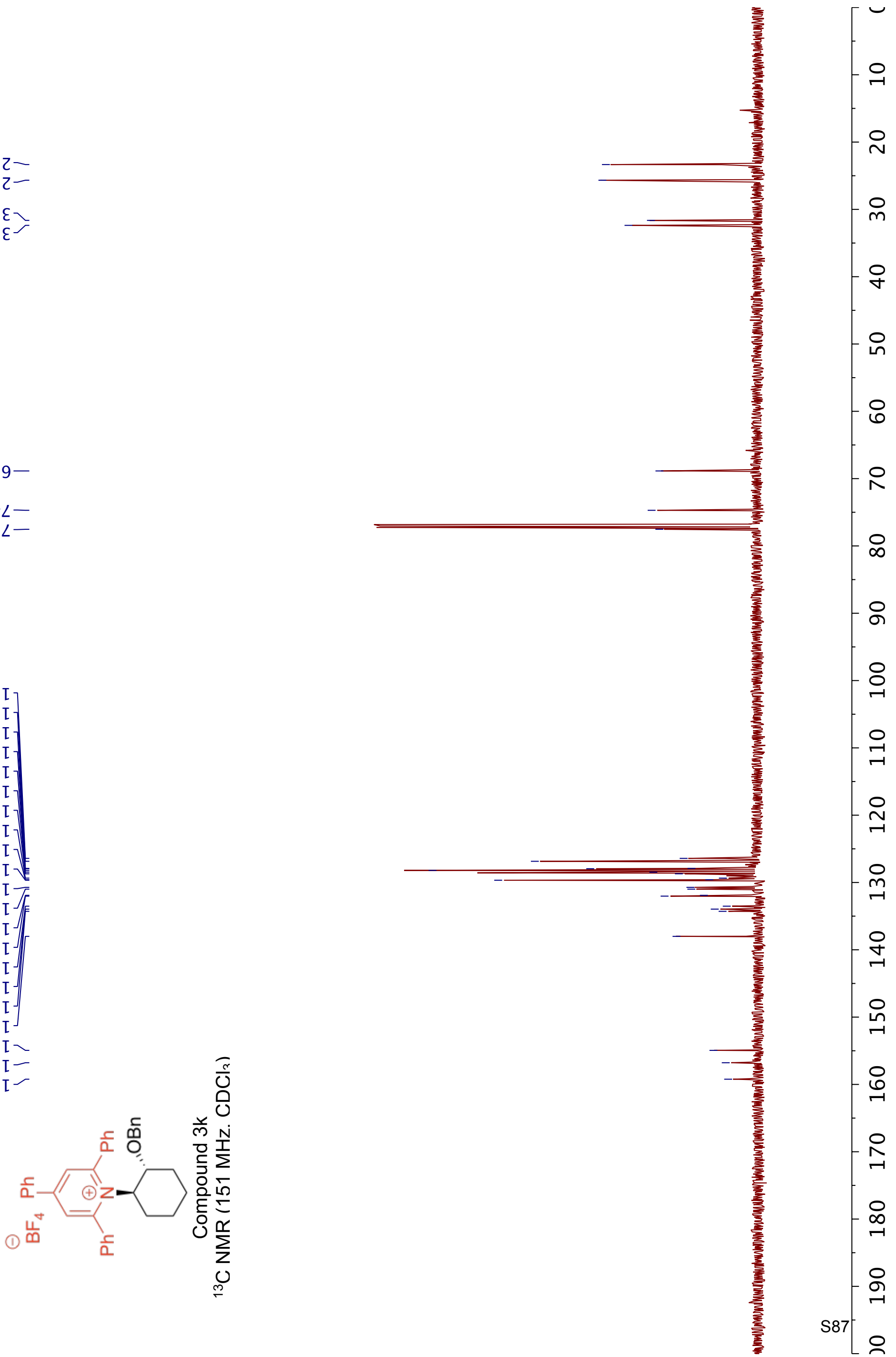


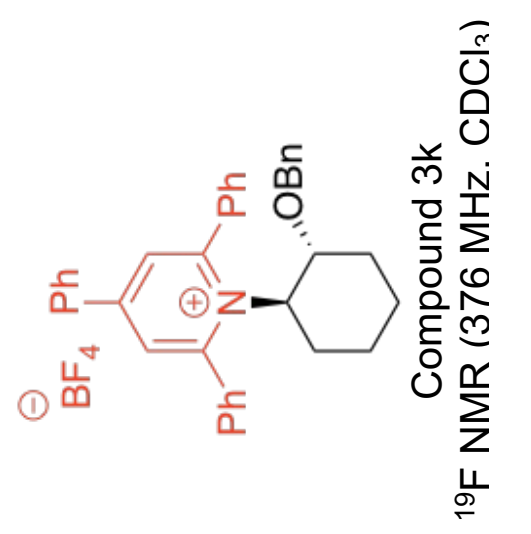

